NUMBER THEORY WEEK 2017

BANACH CENTER PUBLICATIONS, VOLUME 118

INSTITUTE OF MATHEMATICS

POLISH ACADEMY OF SCIENCES

WARSZAWA 2019

\title{
SUPER-POSITIVITY OF A FAMILY OF L-FUNCTIONS
}

\author{
DORIAN GOLDFELD \\ Department of Mathematics, Columbia University \\ New York, NY 10027, USA \\ E-mail: goldfeld@columbia.edu \\ BINGRONG HUANG \\ School of Mathematics, Shandong University \\ Jinan, Shandong 250100, China; \\ School of Mathematical Sciences, Tel Aviv University \\ Tel Aviv, Israel \\ E-mail: bingronghuangsdu@gmail.com
}

This paper is dedicated to Jerzy Kaczorowski on the occasion of his 60th birthday

\begin{abstract}
Zhiwei Yun and Wei Zhang introduced the notion of "super-positivity of self dual L-functions" which specifies that all derivatives of the completed L-function (including Gamma factors and power of the conductor) at the central value $s=1 / 2$ should be non-negative. They proved that the Riemann hypothesis implies super-positivity for self dual cuspidal automorphic L-functions on $G L(n)$. Super-positivity of the Riemann zeta function was established by Pólya in 1927 and since then many other cases have been found by numerical computation. In this paper we prove, for the first time, that there are infinitely many L-functions associated to modular forms for $S L(2, \mathbf{Z})$ each of which has the super-positivity property. Our proof also establishes that all derivatives of the completed L-function at any real point $\sigma>1 / 2$ must be positive.
\end{abstract}

1. Introduction. Let $F$ be a number field and let $\mathbb{A}_{F}$ be the adéle ring of $F$ which is the restricted product $\prod_{v} F_{v}$ over the completions of $F$. A cuspidal automorphic representa-

2010 Mathematics Subject Classification: Primary 11Mxx, 11F11.

Key words and phrases: L-functions, mollification, moments, real zeros, super-positivity, zerodensity.

The paper is in final form and no version of it will be published elsewhere. 
tion $\pi$ of $G L\left(n, \mathbb{A}_{F}\right)$ can be written as a tensor product $\pi=\bigotimes \pi_{v}$ of local representations. Then $\pi$ has a Godement-Jacquet L-function

$$
L(s, \pi)=\prod_{v} L\left(s, \pi_{v}\right)
$$

where

$$
L\left(s, \pi_{v}\right)= \begin{cases}\prod_{j=1}^{n}\left(1-\frac{\alpha_{j}(v)}{N(v)^{s}}\right)^{-1}, & \text { if } v \text { is non-archimedean, } \\ \prod_{j=1}^{n} \Gamma_{v}\left(s-\mu_{j}(v)\right), & \text { if } v \text { is archimedean, }\end{cases}
$$

with $\alpha_{j}(v), \mu_{j}(v) \in \mathbb{C}$ for $j=1,2, \ldots, n$, and

$$
\Gamma_{v}(s)= \begin{cases}\pi^{-s / 2} \Gamma\left(\frac{s}{2}\right), & \text { if } F_{v} \sim \mathbb{R}, \\ (2 \pi)^{-s} \Gamma(s), & \text { if } F_{v} \sim \mathbb{C} .\end{cases}
$$

Let $\tilde{\pi}$ denote the contragredient representation. It is well known that $L(s, \pi)$ is an entire function of order 1 and satisfies a functional equation of the form (see [5, 7])

$$
L(s, \pi)=\epsilon(s, \pi) L(1-s, \tilde{\pi}),
$$

with

$$
\epsilon(s, \pi)=\epsilon(\pi) N_{\pi}^{1 / 2-s},
$$

where $N_{\pi} \geq 1$ is the conductor of $\pi$ and $\epsilon(\pi)$ is the root number satisfying $|\epsilon(\pi)|=1$. If $\pi=\tilde{\pi}$, i.e., $\pi$ is self dual, then $\epsilon(\pi)= \pm 1$.

Zhiwei Yun and Wei Zhang 23] introduced the notion of super-positivity for self dual cuspidal automorphic representations $\pi$ of $G L\left(n, \mathbb{A}_{F}\right)$ which specifies that all derivatives of the completed L-function,

$$
\Lambda(s, \pi):=N_{\pi}^{(s-1 / 2) / 2} L(s, \pi)= \pm \Lambda(1-s, \pi),
$$

at $s=1 / 2$ should be greater or equal to zero. They proved that super-positivity holds for self dual cuspidal automorphic L-functions (over any global field) which satisfy the Riemann hypothesis. In the case that $F$ is a function field, the Riemann hypothesis is known by the theorem of Deligne on Weil's conjecture, and of Drinfeld and Lafforgue on the global Langlands correspondence, so it is now known that super-positivity holds for cuspidal automorphic automorphic representations of $G L\left(n, \mathbb{A}_{F}\right)$ where $F$ is a function field.

Super-positivity was established for the example of the Riemann zeta function by Pólya in 1927 (see [17] and [4]). We would like to thank Peter Sarnak for informing us that super-positivity is also known in many other cases (including quadratic Dirichlet L-functions and L-functions of $G L(2)$ modular forms) when the L-function is "positive definite" as defined by Sarnak [19]. It is not hard to check numerically if an L-function is positive definite or not. For example, in the case of an L-function associated to a holomorphic modular form $f$ for $S L\left(2, \mathbb{Z}\right.$ ) (with Fourier coefficients $a_{n}$ ) it is enough to check if $f(i y)=\sum_{n=1}^{\infty} a_{n} e^{-2 \pi n y}$ is positive for $y \geq 1$.

It was shown by Jung [13] that almost all L-functions in any reasonable family will not be positive definite. It is not known if there are infinitely many self dual automorphic L-functions which are positive definite. 
It seems to be infeasible to prove super-positivity for all Dirichlet L-functions at this time since it would follow that there are no Siegel zeros (real zeros near $s=1$ ) for Dirichlet L-functions, which is known to be a notoriously difficult problem. Similarly, proving super-positivity for all cuspidal automorphic L-functions on $G L\left(n, \mathbb{A}_{F}\right.$ ) (with $F$ $=$ a number field and $n>1$ ) also seems hopeless at present.

The main aim of this paper is to prove that there are infinitely many examples of cuspidal automorphic L-functions for $G L\left(2, \mathbb{A}_{\mathbb{Q}}\right)$ which have the super-positivity property. Following 23 . we will actually prove our results for the following expanded definition of super-positivity.

1.1. Definition (Super-positivity). Fix a number field $F$. Let $\pi$ denote a self dual cuspidal automorphic representation of $G L\left(n, \mathbb{A}_{F}\right)$ with conductor $N_{\pi}$. We say $\pi$ has the super-positivity property if

(1) $\Lambda^{(k)}(1 / 2, \pi)=\left.\left(\frac{d}{d s}\right)^{k} \Lambda(s, \pi)\right|_{s=1 / 2} \geq 0 \quad($ for all $k=0,1,2, \ldots)$,

(2) $\Lambda^{(k)}(\sigma, \pi)=\left.\left(\frac{d}{d s}\right)^{k} \Lambda(s, \pi)\right|_{s=\sigma}>0 \quad($ for $\sigma>1 / 2$ and all $k=0,1,2, \ldots)$,

(3) $\Lambda^{\left(k_{0}\right)}(1 / 2, \pi) \neq 0 \Longrightarrow \Lambda^{\left(k_{0}+2 i\right)}(1 / 2, \pi) \neq 0$ (for some $k_{0} \geq 0$ and all $\left.i=0,1,2, \ldots\right)$.

We now state our main results. Proofs will follow in subsequent sections.

1.2. THEOREM. Let $\pi$ denote a self dual cuspidal automorphic representation of $G L(n, \mathbb{A})$. For $s \in \mathbb{C}$ let $\Lambda(s, \pi)$ be the completed $L$-function of $\pi$ with functional equation

$$
\Lambda(s, \pi)= \pm \Lambda(1-s, \pi) .
$$

Assume that $\Lambda(s, \pi) \neq 0$ for $s=\sigma+i$ where $1 / 2<\sigma<1,|t| \leq \sigma-1 / 2$. Then $\pi$ has the super-positivity property as in Definition 1.1 .

1.3. Remark. The above theorem is essentially due to Stark-Zagier [21, but we will give the short simple proof in $\$ 2$ for the convenience of the reader.

1.4. REMARK. Theorem 1.2 provides a method to manually check if an individual L-function has the super-positivity property. It is enough to check, for example, that all the zeros of $\Lambda(s, \pi)$ with imaginary part $\leq 1 / 2$ are on the line $\operatorname{Re}(s)=1 / 2$. By examining the zeros of L-functions database [16] one sees that all the self dual L-functions in this database do indeed satisfy the super-positivity property.

Next, we apply Theorem 1.2 to show that there is an infinite family of $G L(2)$ L-functions with the super-positivity property. Let $S_{k}$ denote the space of holomorphic cusp forms $f$ of weight $k$ for $S L(2, \mathbb{Z})$. For $f \in S_{k}$, let $L(s, f)$ denote the L-function associated to $f$. For ease of notation, we shall say $L(s, f)$ has the "super-positivity property" if its associated cuspidal automorphic representation has the super-positivity property.

Let $H_{k}$ be the basis of forms of $S_{k}$ that are eigenfunctions of all the Hecke operators. Put

$$
\begin{aligned}
& \mathcal{H}_{+}(K):=\left\{f \in H_{k} \mid K \leq k \leq 2 K, k \equiv 0(\bmod 4)\right\} \\
& \mathcal{H}_{-}(K):=\left\{f \in H_{k} \mid K \leq k \leq 2 K, k \equiv 2(\bmod 4)\right\}
\end{aligned}
$$


1.5. REMARK. For $f \in \mathcal{H}_{+}(K)$, the form $f$ is even, the sign in the functional equation of $L(s, f)$ is positive, and $L(s, f)$ must have a zero of even order at $s=1 / 2$. Similarly for $f \in \mathcal{H}_{-}(K)$ the form $f$ is odd, the sign in the functional equation of $L(s, f)$ is negative, and $L(s, f)$ must have a zero of odd order at $s=1 / 2$.

1.6. TheOREM. The number of $f \in \mathcal{H}_{-}(K)$ such that $L(s, f)$ has no zero in the region $s=\sigma+$ it with $1 / 2<\sigma<1,|t| \leq \sigma-1 / 2$ is $\gg K^{2} / \log K$.

1.7. Remark. The proof of Theorem 1.6 is based on [2], where Conrey and Soundararajan combined Selberg's Lemma 3.2 with the mollification method to prove that a positive proportion of quadratic Dirichlet L-functions have no zeros in $[0,1]$. The proof in [2] requires estimating the total number of zeros for the family of quadratic Dirichlet

L-functions in a thin rectangular region. On the other hand, our proof of Theorem 1.6 uses many such regions to cover the triangle $1 / 2<\sigma<1,|t| \leq \sigma-1 / 2$.

By combining Theorems 1.2, 1.6, we immediately obtain the following.

1.8. THEOREM. There are infinitely many odd modular forms $f$ for $S L(2, \mathbb{Z})$ such that $L(s, f)$ has the super-positivity property. In fact, the number of $f \in \mathcal{H}_{-}(K)$ which have the super-positivity property is $\gg K^{2} / \log K$.

In the course of proving Theorem 1.8 we also obtained the following result as a byproduct.

1.9. THEOREM. There are infinitely many modular forms $f$ for $S L(2, \mathbb{Z})$ such that $L(s, f)$ has no real zeros in the region $\operatorname{Re}(s)>0$ except at $s=1 / 2$. In fact, the number of $f \in \mathcal{H}_{-}(K)$ (respectively $f \in \mathcal{H}_{+}(K)$ ) with this property is $\gg K^{2} / \log K$.

1.10. Remark. We learned from Ricotta [18, p. 292] that in 2003, Soundarajaran announced a result similar to Theorem 1.9 for $\mathcal{H}_{+}(K)$ and $\mathcal{H}_{-}(K)$. Recently, Soundararajan informed us that he and Conrey proved that for $K$ large, at least $71 \%$ of $f \in \mathcal{H}_{-}(K)$ and at least $38 \%$ of $f \in \mathcal{H}_{+}(K)$ satisfy Theorem 1.9 However, they never published this result. The proof for the case $\mathcal{H}_{-}(K)$ is given in $\$ 7$ because it is needed for the proof of Theorem 1.8. This result is not optimal since we did not try to remove the harmonic weight and our choice of the mollifier is not optimal. One may also give a similar proof for $\mathcal{H}_{+}(K)$, but it is omitted here since this is not needed for our main results.

Although we focussed on the family of Hecke cusp forms $H_{k}$ for $S L(2, \mathbb{Z})$, the methods introduced in this paper can also be applied to other families of automorphic forms of varying level, weight and spectrum. We plan to continue these investigations in a future research paper.

\section{Proof of Theorem 1.2, Let}

$$
\lambda(s, \pi):=\Lambda(s+1 / 2, \pi) .
$$

Then, as in [23], the function $\lambda(s, \pi)$ has the following properties:

- $\lambda(-s)= \pm \lambda(s)$

- $\lambda(\sigma)>0$ for $\sigma>1 / 2$, 
- $\lambda(s)$ is entire and of order 1 ,

- if $\lambda\left( \pm \rho_{k}\right)=0$ (with $\rho_{k} \neq 0, \operatorname{Im} \rho_{k} \geq 0$ ) for $k=1,2, \ldots$ (denotes the non-central zeros of $\lambda(s)$ ), then for some integer $m \geq 0$ and $A>0$,

$$
\lambda(s)=s^{m} e^{A} \prod_{k=1}^{\infty}\left(1-\frac{s^{2}}{\rho_{k}^{2}}\right) \quad \text { (Hadamard Product Formula). }
$$

Now, let $\beta+i \gamma$ be a non-trivial zero of $\lambda(s)$ with $\beta, \gamma \in \mathbb{R}$. Then by our assumptions either

- $\beta=0$ and there are two zeros of $\lambda(s)$ at $s= \pm i \gamma$;

- $\beta>0,|\gamma|>\beta$, and there are four zeros of $\lambda(s)$ at $s=\beta+i \gamma,-\beta+i \gamma, \beta-i \gamma$, $-\beta-i \gamma$.

Consequently, we may rewrite 2.1 in the form

$$
\lambda(s)=s^{m} e^{A} \prod_{\substack{\lambda(\beta+i \gamma)=0 \\ \beta>0 \text { and }|\gamma|>\beta}}\left(1+\frac{\left(2 \gamma^{2}-2 \beta^{2}\right) s^{2}+s^{4}}{\left(\gamma^{2}+\beta^{2}\right)^{2}}\right) \prod_{\substack{\lambda(\beta+i \gamma)=0 \\ \beta=0}}\left(1+\frac{s^{2}}{\gamma^{2}}\right) .
$$

It immediately follows from 2.2 that all derivatives of $\lambda(s)$ at $s=0$ must be greater than or equal to zero and all derivatives of $\lambda(s)$ at $s=\sigma>1 / 2$ must be positive. Condition (3) of Definition 1.1 for super-positivity of $\pi$ follows as in [23].

\section{Requisite background material needed for the proof of Theorem $\mathbf{1 . 6}$}

3.1. Selberg's Lemma. We will need the following version of the argument principle, which is due to Selberg.

3.2. Lemma. For $W \in \mathbb{R}$, let $\phi(s)$ be a holomorphic function of a complex variable $s$ that does not vanish on a half-plane $\operatorname{Re}(s) \geq W$. Let $\mathcal{B}$ be the rectangular box of vertices $W_{0} \pm i H, W_{1} \pm i H$, where $H>0$ and $W_{0}<W<W_{1}$. Then

$$
\begin{aligned}
4 H \sum_{\substack{\beta+i \gamma \in \mathcal{B} \\
\phi(\beta+i \gamma)=0}} \cos \left(\frac{\pi \gamma}{2 H}\right) \sinh & \left(\frac{\pi\left(\beta-W_{0}\right)}{2 H}\right) \\
= & \int_{-H}^{H} \cos \left(\frac{\pi t}{2 H}\right) \log \left|\phi\left(W_{0}+i t\right)\right| d t \\
& +\int_{W_{0}}^{W_{1}} \sinh \left(\frac{\pi\left(\alpha-W_{0}\right)}{2 H}\right) \log |\phi(\alpha+i H) \phi(\alpha-i H)| d \alpha \\
& -\operatorname{Re}\left(\int_{-H}^{H} \cos \left(\frac{\pi\left(W_{1}-W_{0}+i t\right)}{2 i H}\right)(\log \phi)\left(W_{1}+i t\right) d t\right) .
\end{aligned}
$$

Proof. See Selberg [20, Lemma 14] or Conrey-Soundararajan [2, Lemma 2.1]. 


\subsection{An average of the $J$-Bessel function}

3.4. Lemma. Suppose $\Phi \in C_{0}^{\infty}\left(\mathbb{R}^{+}\right)$is a real valued function and $K \geq 1$. For $x>0$, we have

$$
\begin{aligned}
4 \sum_{k \equiv 2(4)} \Phi\left(\frac{k-1}{K}\right) J_{k-1}(x) \\
\quad=\Phi\left(\frac{x}{K}\right)+\frac{K}{\sqrt{x}} \operatorname{Im}\left(e^{-2 \pi i / 8} e^{i x} \check{\Phi}\left(\frac{K^{2}}{2 x}\right)\right)+\mathcal{O}\left(\frac{x}{K^{3}} \int_{-\infty}^{\infty}|v|^{3}|\hat{\Phi}(v)| d v\right),
\end{aligned}
$$

where

$$
\breve{\Phi}(v):=\int_{0}^{\infty} \frac{\Phi(\sqrt{u})}{\sqrt{2 \pi u}} e^{i u v} d u,
$$

and $\hat{\Phi}$ is the Fourier transform of $\Phi$. The implied constant is absolute.

Proof. See Iwaniec [10, Lemma 5.8], Iwaniec-Luo-Sarnak [12, Proposition 8.1], and Khan [14, Lemma 2.3].

\subsection{The approximate functional equation. Let}

$$
f(z)=\sum_{n=1}^{\infty} \lambda_{f}(n)(4 \pi n)^{(k-1) / 2} e^{2 \pi i n z}
$$

(for $z$ in the upper half plane) be a modular form of weight $k$ for $S L(2, \mathbb{Z})$ with associated L-function

$$
L(s, f):=\sum_{n=1}^{\infty} \frac{\lambda_{f}(n)}{n^{s}} \quad(\operatorname{Re}(s)>1) .
$$

Fix a smooth function $H: \mathbb{R}^{+} \rightarrow \mathbb{R}^{+}$satisfying $H(x)=1$ for $x \in[0,1 / 2]$, and $H(x)+H(1 / x)=1$ for $x \in \mathbb{R}^{+}$. We know the Mellin transform $\widetilde{H}(s)=\int_{0}^{\infty} H(y) y^{s} \frac{d y}{y}$ has a single simple pole at 0 of residue 1 , and is odd. Furthermore, $\widetilde{H}(s)$ satisfies the bounds $\widetilde{H}(s) \ll A \frac{1}{|s(s+1) \cdots(s+A-1)|}, A=1,2, \ldots$, and $\widetilde{H}(s) \ll 2^{\operatorname{Re}(s)}$ for $\operatorname{Re}(s)>1$.

3.6. Lemma. Let $K \geq 1$, and $k \asymp K$ an even integer. Let $-\frac{B}{\log K} \leq \delta \leq \vartheta$ and $t \ll K$. Then for any modular form $f$ of weight $k$ for $S L(2, \mathbb{Z})$, we have

$$
|L(1 / 2+\delta+i t, f)|^{2}=\sum_{d=1}^{\infty} \frac{1}{d^{1+2 \delta}} \sum_{n=1}^{\infty} \frac{\lambda_{f}(n) \eta_{i t}(n)}{n^{1 / 2+\delta}} V_{k, \delta+i t}\left(n d^{2}\right) .
$$

Here $\eta_{\nu}(n):=\sum_{a d=n}\left(\frac{a}{d}\right)^{\nu}$ is the generalized divisor function, and for any $y>0$,

$$
V_{k, \delta+i t}(y):=\frac{1}{2 \pi i} \int_{3-i \infty}^{3+i \infty} \frac{\widetilde{H}(s+\delta)+\widetilde{H}(s-\delta)}{\left(4 \pi^{2} y\right)^{s-\delta}} \frac{\Gamma(s+k / 2+i t) \Gamma(s+k / 2-i t)}{\Gamma(\delta+k / 2+i t) \Gamma(\delta+k / 2-i t)} d s
$$

is real valued, and satisfies the following:

$$
\begin{aligned}
& V_{k, \delta+i t}(y)=1+\left(4 \pi^{2} y\right)^{2 \delta} \frac{\Gamma(-\delta+k / 2+i t) \Gamma(-\delta+k / 2-i t)}{\Gamma(\delta+k / 2+i t) \Gamma(\delta+k / 2-i t)}+\mathcal{O}_{A}\left(\left(\frac{y}{k^{2}}\right)^{A}\right), \\
& V_{k, \delta+i t}(y) \ll_{A}\left(\frac{k^{2}}{y}\right)^{A}, \quad y^{j} V_{k, \delta+i t}^{(j)}(y) \ll_{j} 1,
\end{aligned}
$$


for $A>0$ and any integer $j \geq 0$. We also have

$$
V_{k, \delta+i t}(y)=\frac{1}{2 \pi i} \int_{\alpha-i \infty}^{\alpha+i \infty} \frac{\widetilde{H}(s+\delta)+\widetilde{H}(s-\delta)}{\left(16 \pi^{2}\right)^{s-\delta}}\left(\frac{k^{2}}{y}\right)^{s-\delta} d s+\mathcal{O}_{\varepsilon}\left(|t|^{2} y^{-\varepsilon} k^{-1+\varepsilon}\right)
$$

for any $\alpha>|\delta|$.

Proof. See Iwaniec-Kowalski [11, pp. 97-100] and Hough [9, Proposition 3.7].

3.7. The Petersson trace formula. Each Hecke eigenform $f \in H_{k}$ has a Fourier expansion

$$
f(z)=\sum_{n=1}^{\infty} \lambda_{f}(n)(4 \pi n)^{(k-1) / 2} e^{2 \pi i n z} \quad(z \in \mathbb{C}, \operatorname{Im}(z)>0),
$$

where $\lambda_{f}(n) \in \mathbb{R}$ for $n=1,2, \ldots$ We normalize $f$ by setting $\lambda_{f}(1)=1$. The Fourier coefficients of $f$ satisfy the relation

$$
\lambda_{f}(m) \lambda_{f}(n)=\sum_{d \mid(m, n)} \lambda_{f}\left(\frac{m n}{d^{2}}\right) .
$$

The Petersson trace formula is given by the following basic orthogonality relation on $H_{k}$.

3.9. Lemma. Let $m, n \geq 1$. Then

$$
\sum_{f \in H_{k}} \omega_{f} \cdot \lambda_{f}(m) \lambda_{f}(n)=\delta_{m, n}+2 \pi i^{-k} \sum_{c=1}^{\infty} \frac{S(m, n ; c)}{c} J_{k-1}\left(\frac{4 \pi \sqrt{m n}}{c}\right) .
$$

where

$$
\omega_{f}=\frac{12 \zeta(2)}{(k-1)} \cdot \frac{1}{L\left(1, \operatorname{sym}^{2} f\right)}
$$

is termed the harmonic weight of $f$.

Proof. See e.g. Iwaniec [10, Theorem 3.6] and [1, §2.1].

By appealing to the well known estimate $J_{k-1}(x) \ll \min \left(x^{k-1}, x^{-1 / 2}\right)$ it easily follows that

$$
\sum_{f \in H_{k}} \omega_{f}=1+\mathcal{O}\left(2^{-k}\right)
$$

3.11. The Voronoi summation formula for Eisenstein series. Let $\nu \in \mathbb{C}$. The generalized divisor function

$$
\eta_{\nu}(n)=\sum_{a d=n}\left(\frac{a}{d}\right)^{\nu}
$$

occurs in the Fourier expansion of Eisenstein series. We have the following version of the Voronoi summation formula. 
3.12. Lemma. Let $g: \mathbb{R}^{+} \rightarrow \mathbb{R}^{+}$be a smooth and compactly supported function. Let $c \geq 1$ and $(a, c)=1$ with $a d \equiv 1(c)$. Then

$$
\begin{aligned}
\sum_{n=1}^{\infty} \eta_{i t}(n) g(n) e^{2 \pi i a n / c}= & c^{2 i t-1} \zeta(1-2 i t) \int_{0}^{\infty} g(x) x^{-i t} d x \\
& +c^{-2 i t-1} \zeta(1+2 i t) \int_{0}^{\infty} g(x) x^{i t} d x \\
& +\frac{1}{c} \sum_{n=1}^{\infty} \eta_{i t}(n) e\left(-\frac{d n}{c}\right) \int_{0}^{\infty} g(x) J_{2 i t}^{+}\left(\frac{4 \pi \sqrt{n x}}{c}\right) d x \\
& +\frac{1}{c} \sum_{n=1}^{\infty} \eta_{i t}(n) e\left(\frac{d n}{c}\right) \int_{0}^{\infty} g(x) K_{2 i t}^{+}\left(\frac{4 \pi \sqrt{n x}}{c}\right) d x,
\end{aligned}
$$

where

$$
J_{\nu}^{+}(x):=\frac{-\pi}{\sin (\pi \nu / 2)}\left(J_{\nu}(x)-J_{-\nu}(x)\right), \quad K_{\nu}^{+}(x):=4 \cos \frac{\pi \nu}{2} K_{\nu}(x) .
$$

Proof. See e.g. Hough [9, Lemma 3.3].

4. The twisted second moment near the critical point. Recall that $H_{k}$ denotes a basis for the space of holomorphic Hecke cusp forms of weight $k \geq 12$ for $S L(2, \mathbb{Z})$. Let $\mathcal{H}=\bigcup_{k} H_{k}$. Assume that for all $f \in \mathcal{H}$ there is some uniquely defined $\alpha_{f} \in \mathbb{C}$. Consider the set

$$
\left\{\alpha_{f}\right\}:=\left\{\alpha_{f}\right\}_{f \in \mathcal{H}} .
$$

The basic objects of study for the rest of this paper are given in the following definition.

4.1. Definition. Let

$$
\omega_{f}:=\frac{12 \zeta(2)}{(k-1)} \cdot \frac{1}{L\left(1, \operatorname{sym}^{2} f\right)}
$$

denote the harmonic weight of $f \in H_{k}$. Let $\Phi: \mathbb{R} \rightarrow \mathbb{R}_{\geq 0}$ be a fixed smooth non-negative function supported on $[1,2]$ and let

$$
\mathcal{R}_{1 / 2}:=\{\beta+i \gamma \mid \beta \in(1 / 2,1) \text { and }|\gamma| \leq \beta-1 / 2\} .
$$

For $K>0$, define the following sums

$$
\begin{aligned}
\mathcal{A}\left(\left\{\alpha_{f}\right\} ; K, \Phi\right) & :=\sum_{k \equiv 2(4)} \Phi\left(\frac{k-1}{K}\right) \sum_{f \in H_{k}} \omega_{f} \cdot \alpha_{f}, \\
\mathcal{A}(K, \Phi) & :=\sum_{k \equiv 2(4)} \Phi\left(\frac{k-1}{K}\right) \sum_{f \in H_{k}} \omega_{f}, \\
\mathcal{M}(K, \Phi) & :=\sum_{k \equiv 2(4)} \Phi\left(\frac{k-1}{K}\right) \sum_{\substack{f \in H_{k} \\
L(s, f) \neq 0 \text { for } s \in \mathcal{R}_{1 / 2}}} \omega_{f}, \\
\mathcal{N}(K, \Phi) & :=\sum_{k \equiv 2(4)} \Phi\left(\frac{k-1}{K}\right) \sum_{\substack{f \in H_{k} \\
L(s, f) \text { has at least } \\
\text { one zero in } \mathcal{R}_{1 / 2}}} \omega_{f} .
\end{aligned}
$$


It is clear that

$$
\mathcal{M}(K ; \Phi)+\mathcal{N}(K ; \Phi)=\mathcal{A}(K ; \Phi) .
$$

The key strategy for proving Theorem 1.6 is to try to show that $\mathcal{M}(K, \Phi)$ is large compared to $\mathcal{A}(K, \Phi)$. To achieve this goal we will use the mollification method which leads us to first consider the following twisted second moment of $L(s, f)$ at the special value $s=1 / 2+\delta+i t$.

4.2. Theorem. Let $-\frac{B}{\log K} \leq \delta \leq \vartheta$ and $t \ll K^{\theta}$, with $0<2 \theta \leq \vartheta \leq 1 / 100$ two small positive constants. Let $\ell \leq K^{2-4 \vartheta}$. We have the following asymptotic formula.

$$
\begin{aligned}
\mathcal{A}( & \left.\left\{\lambda_{f}(\ell)|L(1 / 2+\delta+i t, f)|^{2}\right\} ; K, \Phi\right) \\
= & \zeta(1+2 \delta) \frac{\eta_{i t}(\ell)}{\ell^{1 / 2+\delta}} \frac{K}{4} \int_{0}^{\infty} \Phi(u) d u+\zeta(1-2 \delta) \frac{\eta_{i t}(\ell)}{\ell^{1 / 2-\delta}}\left(\frac{K}{4 \pi}\right)^{-4 \delta} \frac{K}{4} \int_{0}^{\infty} \Phi(u) u^{-4 \delta} d u \\
& -2 \operatorname{Re}\left\{\zeta(1+2 i t) \frac{\eta_{\delta}(\ell)}{\ell^{1 / 2+i t}}\left(\frac{K}{4 \pi}\right)^{-2 \delta+2 i t} \frac{K}{4} \int_{0}^{\infty} \Phi(u) u^{-2 \delta+2 i t} d u\right\} \\
& +\mathcal{O}_{\varepsilon}\left((1+|t|)^{2} \ell^{-\delta} K^{\varepsilon}+(1+|t|)^{4} \ell^{1 / 2} K^{-1+\varepsilon}\right) .
\end{aligned}
$$

Proof. From the approximate functional equation, we have

$$
\begin{aligned}
\mathcal{A}\left(\left\{\lambda_{f}(\ell) \mid\right.\right. & \left.\left.\left.L(1 / 2+\delta+i t, f)\right|^{2}\right\} ; K, \Phi\right) \\
= & \sum_{k \equiv 2(4)} \Phi\left(\frac{k-1}{K}\right) \sum_{f \in H_{k}} \omega_{f} \cdot \lambda_{f}(\ell)|L(1 / 2+\delta+i t, f)|^{2} \\
& =\sum_{k \equiv 2(4)} \Phi\left(\frac{k-1}{K}\right) \sum_{d=1}^{\infty} \sum_{n=1}^{\infty} \frac{\eta_{i t}(n)}{d^{1+2 \delta} n^{1 / 2+\delta}} V_{k, \delta+i t}\left(n d^{2}\right) \sum_{f \in H_{k}} \omega_{f} \cdot \lambda_{f}(\ell) \lambda_{f}(n) .
\end{aligned}
$$

Applying the Petersson trace formula we obtain

$$
\mathcal{A}\left(\left\{\lambda_{f}(\ell)|L(1 / 2+\delta+i t, f)|^{2}\right\} ; K, \Phi\right)=\mathcal{D}+\mathcal{F},
$$

where we have the diagonal term

$$
\mathcal{D}:=\frac{\eta_{i t}(\ell)}{\ell^{1 / 2+\delta}} \sum_{d=1}^{\infty} \frac{1}{d^{1+2 \delta}} \sum_{k \equiv 2(4)} \Phi\left(\frac{k-1}{K}\right) V_{k, \delta+i t}\left(\ell d^{2}\right),
$$

and the off-diagonal term

$$
\begin{aligned}
\mathcal{F}:= & -2 \pi \sum_{d=1}^{\infty} \sum_{n=1}^{\infty} \frac{\eta_{i t}(n)}{d^{1+2 \delta} n^{1 / 2+\delta}} \sum_{c=1}^{\infty} \frac{S(n, \ell ; c)}{c} \\
& \times \sum_{k \equiv 2(4)} \Phi\left(\frac{k-1}{K}\right) V_{k, \delta+i t}\left(n d^{2}\right) J_{k-1}\left(\frac{4 \pi \sqrt{\ell n}}{c}\right) .
\end{aligned}
$$

4.4. The diagonal term. From now on, we let $\vartheta$ be a fixed positive real number less than $1 / 100$. We first handle the case $-\frac{B}{\log K} \leq \delta \leq \vartheta$ and $\delta \neq 0$. Note that for the 
remaining case $\delta=0$, we can just view it as the limitation of $\delta \rightarrow 0$. Introducing the integral defining $V$, we have

$$
\begin{aligned}
\mathcal{D}= & \frac{\eta_{i t}(\ell)}{\ell^{1 / 2+\delta}} \frac{1}{2 \pi i} \int_{\delta+\varepsilon-i \infty}^{\delta+\varepsilon+i \infty} \frac{\zeta(1+2 s)}{\left(4 \pi^{2} \ell\right)^{s-\delta}}[\widetilde{H}(s-\delta)+\widetilde{H}(s+\delta)] \\
& \times \sum_{k \equiv 2(4)} \Phi\left(\frac{k-1}{K}\right) \frac{\Gamma(s+k / 2+i t) \Gamma(s+k / 2-i t)}{\Gamma(\delta+k / 2+i t) \Gamma(\delta+k / 2-i t)} d s .
\end{aligned}
$$

Since we have

$$
\left|\frac{\Gamma(s+k / 2+i t) \Gamma(s+k / 2-i t)}{\Gamma(\delta+k / 2+i t) \Gamma(\delta+k / 2-i t)}\right| \ll \frac{\Gamma(\delta+\varepsilon+k / 2) \Gamma(\delta+\varepsilon+k / 2)}{\Gamma(\delta+k / 2) \Gamma(\delta+k / 2)} \ll k^{2 \varepsilon}
$$

for $\operatorname{Re}(s)=\delta+\varepsilon$ and rapid decay of $\widetilde{H}(s-\delta)+\widetilde{H}(s+\delta)$ on the vertical line $\operatorname{Re}(s)=\delta+\varepsilon$, we can restrict the integral above to $|\operatorname{Im}(s)| \leq K^{\varepsilon}$ with an error of $O_{\varepsilon, B}\left(\ell^{-1 / 2-\delta} K^{-B}\right)$. For $\operatorname{Re}(s)=\delta+\varepsilon$ and $|\operatorname{Im}(s)| \leq K^{\varepsilon}$, it follows from Stirling's formula that

$$
\frac{\Gamma(z+u)}{z}=z^{u}\left(1+\mathcal{O}\left(\frac{|u|^{2}}{|z|}\right)\right)
$$

so we have (recalling that $t \ll K^{\theta}$ )

$$
\frac{\Gamma(s+k / 2+i t) \Gamma(s+k / 2-i t)}{\Gamma(\delta+k / 2+i t) \Gamma(\delta+k / 2-i t)}=\left(\frac{k-1}{2}\right)^{2(s-\delta)}\left(1+\mathcal{O}\left((1+|t|)^{2} k^{-1+2 \varepsilon}\right)\right)
$$

and then, together with Poisson summation formula (cf. Iwaniec-Kowalski [11, Eq.(4.24)]) we obtain

$$
\begin{aligned}
\sum_{k \equiv 2(4)} \Phi\left(\frac{k-1}{K}\right) \frac{\Gamma(s+k / 2+i t) \Gamma(s+k / 2-i t)}{\Gamma(\delta+k / 2+i t) \Gamma(\delta+k / 2-i t)} \\
=\sum_{k \equiv 2(4)} \Phi\left(\frac{k-1}{K}\right)\left(\frac{k-1}{2}\right)^{2(s-\delta)}\left(1+\mathcal{O}\left((1+|t|)^{2} k^{-1+2 \varepsilon}\right)\right) \\
=\left(\frac{K}{2}\right)^{2(s-\delta)} \sum_{k \equiv 2(4)} \Phi\left(\frac{k-1}{K}\right)\left(\frac{k-1}{K}\right)^{2(s-\delta)}+\mathcal{O}\left((1+|t|)^{2} K^{4 \varepsilon}\right) \\
=\left(\frac{K}{2}\right)^{2(s-\delta)}\left(\frac{K}{4} \int_{0}^{\infty} \Phi(u) u^{2(s-\delta)} d u+O_{B}\left(K^{-B}\right)\right)+\mathcal{O}\left((1+|t|)^{2} K^{4 \varepsilon}\right) \\
=\left(\frac{K}{2}\right)^{2(s-\delta)} \frac{K}{4} \int_{0}^{\infty} \Phi(u) u^{2(s-\delta)} d u+\mathcal{O}\left((1+|t|)^{2} K^{4 \varepsilon}\right) .
\end{aligned}
$$

Hence

$$
\begin{aligned}
\mathcal{D}= & \frac{\eta_{i t}(\ell)}{\ell^{1 / 2+\delta}} \frac{1}{2 \pi i} \int_{\delta+\varepsilon-i K^{\varepsilon}}^{\delta+\varepsilon+i K^{\varepsilon}} \frac{\zeta(1+2 s)}{\left(4 \pi^{2} \ell\right)^{s-\delta}}[\widetilde{H}(s-\delta)+\widetilde{H}(s+\delta)] \\
& \times\left[\left(\frac{K}{2}\right)^{2(s-\delta)} \frac{K}{4} \int_{0}^{\infty} \Phi(u) u^{2(s-\delta)} d u+\mathcal{O}\left((1+|t|)^{2} K^{4 \varepsilon}\right)\right] d s \\
& +\mathcal{O}_{\varepsilon, B}\left(\ell^{-1 / 2-\delta} K^{-B}\right)
\end{aligned}
$$




$$
\begin{aligned}
= & \frac{\eta_{i t}(\ell)}{\ell^{1 / 2+\delta}} \frac{K}{4} \frac{1}{2 \pi i} \int_{\delta+\varepsilon-i K^{\varepsilon}}^{\delta+\varepsilon+i K^{\varepsilon}} \frac{\zeta(1+2 s)}{\left(4 \pi^{2} \ell\right)^{s-\delta}}[\widetilde{H}(s-\delta)+\widetilde{H}(s+\delta)] \\
& \times\left(\frac{K}{2}\right)^{2(s-\delta)} \int_{0}^{\infty} \Phi(u) u^{2(s-\delta)} d u d s+\mathcal{O}_{\varepsilon}\left((1+|t|)^{2} \ell^{-1 / 2-\delta} K^{4 \varepsilon}\right) \\
& +\mathcal{O}_{\varepsilon, B}\left(\ell^{-1 / 2-\delta} K^{-B}\right) \\
= & \frac{\eta_{i t}(\ell)}{\ell^{1 / 2+\delta}} \frac{K}{4} \frac{1}{2 \pi i} \int_{\delta+\varepsilon-i \infty}^{\delta+\varepsilon-i \infty} \frac{\zeta(1+2 s)}{\left(4 \pi^{2} \ell\right)^{s-\delta}}[\widetilde{H}(s-\delta)+\widetilde{H}(s+\delta)] \\
& \times\left(\frac{K}{2}\right)^{2(s-\delta)} \int_{0}^{\infty} \Phi(u) u^{2(s-\delta)} d u d s \\
& +\mathcal{O}_{\varepsilon}\left((1+|t|)^{2} \ell^{-1 / 2-\delta} K^{4 \varepsilon}\right) .
\end{aligned}
$$

By shifting the contour to the line $\operatorname{Re}(s)=-1 / 2+\delta+\varepsilon$, we obtain

$$
\begin{aligned}
\mathcal{D}= & \zeta(1+2 \delta) \frac{\eta_{i t}(\ell)}{\ell^{1 / 2+\delta}} \frac{K}{4} \int_{0}^{\infty} \Phi(u) d u \\
& +\zeta(1-2 \delta) \frac{\eta_{i t}(\ell)}{\ell^{1 / 2-\delta}}\left(\frac{K}{4 \pi}\right)^{-4 \delta} \frac{K}{4} \int_{0}^{\infty} \Phi(u) u^{-4 \delta} d u \\
& +\mathcal{O}\left(\frac{\tau(\ell) K}{\ell^{1 / 2+\delta}} \mid \int_{-1 / 2+\delta+\varepsilon-i \infty}^{-1 / 2+\delta+\varepsilon+i \infty} \frac{\zeta(1+2 s)}{(4 \pi \ell)^{s-\delta}}[\widetilde{H}(s-\delta)+\widetilde{H}(s+\delta)]\right. \\
& \left.\times\left(\frac{K}{2}\right)^{2(s-\delta)} \int_{0}^{\infty} \Phi(u) u^{2(s-\delta)} d u d s \mid\right)+\mathcal{O}\left((1+|t|)^{2} \ell^{-1 / 2-\delta} K^{4 \varepsilon}\right) \\
= & \zeta(1+2 \delta) \frac{\eta_{i t}(\ell)}{\ell^{1 / 2+\delta}} \frac{K}{4} \int_{0}^{\infty} \Phi(u) d u \\
& +\zeta(1-2 \delta) \frac{\eta_{i t}(\ell)}{\ell^{1 / 2-\delta}}\left(\frac{K}{4 \pi}\right)^{-4 \delta} \frac{K}{4} \int_{0}^{\infty} \Phi(u) u^{-4 \delta} d u \\
& +\mathcal{O}_{\varepsilon}\left((1+|t|)^{2} \ell^{-\delta} K^{4 \varepsilon}\right) .
\end{aligned}
$$

4.6. The off-diagonal term. We assume $t \neq 0$. The case $t=0$, can be viewed as the limitation of the case $t \neq 0$. Of course, we can also use the Voronoi summation formula for $\tau(n)$, the divisor function (cf. Iwaniec-Kowalski [11, Eq. (4.49)]) instead of Lemma 3.12 to do the estimation. Note that $\left|J_{k}(x)\right| \leq(x / 2)^{k-1}$ for $k>4$, and $0<x<1$. By Lemma 3.6 . we can truncate the sums over $c$ and $d$ in $\mathcal{F}$ at $c d \leq \sqrt{\ell} K^{1+\varepsilon}$ with a negligible error. (Indeed, to prove this we consider two cases depending on the size of $n d^{2}$. For the case $n d^{2}>K^{2+\varepsilon}$, by Lemma 3.6 , we know $V_{k, \delta+i t}\left(n d^{2}\right) \ll \frac{1}{n^{2} d^{4}} K^{-B}$; and then we just use the uniform bound $J_{k-1}\left(\frac{4 \pi \sqrt{\ell n}}{c}\right) \ll k^{-1 / 3}$ when $c \leq 4 \pi \sqrt{\ell n}$, and we use the bound $J_{k-1}\left(\frac{4 \pi \sqrt{\ell n}}{c}\right) \ll \frac{\ell n}{c^{2}}$ when $c>4 \pi \sqrt{\ell n}$, so we can bound the total contribution by $K^{-B^{\prime}}$. For the case $n d^{2} \leq K^{2+\varepsilon}$, since $c d \geq \sqrt{\ell} K^{1+\varepsilon}$, we have $\frac{4 \pi \sqrt{\ell n}}{c} \ll K^{-\varepsilon / 2}$. So we have $J_{k-1}\left(\frac{4 \pi \sqrt{\ell n}}{c}\right) \ll \frac{\ell n}{c^{2}} K^{-B}$, and again the contribution to $\mathcal{F}$ will be bounded by $K^{-B^{\prime}}$.) 
Let $x=\frac{4 \pi \sqrt{\ell n}}{c}$. By Lemma 3.4 , we have

$$
\begin{aligned}
4 \sum_{k \equiv 2(4)} \Phi\left(\frac{k-1}{K}\right) & V_{k, \delta+i t}\left(n d^{2}\right) J_{k-1}(x)=\Phi\left(\frac{x}{K}\right) V_{x+1, \delta+i t}\left(n d^{2}\right) \\
& +\frac{K}{\sqrt{x}} \operatorname{Im}\left(e^{-2 \pi i / 8} e^{i x} \int_{0}^{\infty} \frac{\Phi(\sqrt{u})}{\sqrt{2 \pi u}} V_{\sqrt{u} K+1, \delta+i t}\left(n d^{2}\right) e^{i u K^{2} /(2 x)} d u\right) \\
& +\mathcal{O}\left(\frac{x}{K^{3}} \int_{-\infty}^{\infty}|v|^{3}\left|\int_{0}^{\infty} \Phi(u) V_{u K+1, \delta+i t}\left(n d^{2}\right) e^{i u v} d u\right| d v\right)
\end{aligned}
$$

Hence

$$
\mathcal{F}=\mathcal{F}_{1}+\mathcal{F}_{2}+\mathcal{E}+\mathcal{O}\left(K^{-B}\right)
$$

where

$$
\begin{aligned}
\mathcal{F}_{1}:= & -\frac{\pi}{2} \sum_{c d \leq \sqrt{\ell} K^{1+\varepsilon}} \frac{1}{c d^{1+2 \delta}} \sum_{\substack{a=1 \\
(a, c)=1}}^{c} e^{2 \pi i a \ell / c} \sum_{n=1}^{\infty} \frac{\eta_{i t}(n) e^{2 \pi i \bar{a} n / c}}{n^{1 / 2+\delta}} \\
& \times \Phi\left(\frac{4 \pi \sqrt{\ell n}}{c K}\right) V_{4 \pi \sqrt{\ell n} / c+1, \delta+i t}\left(n d^{2}\right), \\
\mathcal{F}_{2}:= & -\frac{\pi^{1 / 2} K}{4 \ell^{1 / 4}} \sum_{c d \leq \sqrt{\ell} K^{1+\varepsilon}} \frac{1}{c^{1 / 2} d^{1+2 \delta}} \sum_{\substack{a=1 \\
(a, c)=1}}^{c} e^{2 \pi i a \ell / c} \sum_{n=1}^{\infty} \frac{\eta_{i t}(n) e^{2 \pi i \bar{a} n / c}}{n^{3 / 4+\delta}} \\
& \times \operatorname{Im}\left(e^{-2 \pi i / 8} e^{i 4 \pi \sqrt{\ell n} / c} \int_{0}^{\infty} \frac{\Phi(\sqrt{u})}{\sqrt{2 \pi u}} V_{\sqrt{u} K+1, \delta+i t}\left(n d^{2}\right) e^{i u c K^{2} /(8 \pi \sqrt{\ell n})} d u\right), \\
& \ll \frac{\ell^{1 / 2}}{K^{3}} \sum_{d=1}^{\infty} \sum_{n=1}^{\infty} \frac{\tau(n)}{d^{1+2 \delta} n^{\delta}} \sum_{c \leq \sqrt{\ell} K^{1+\varepsilon} / d} \frac{|S(n, \ell ; c)|}{c^{2}} \\
& \times \int_{-\infty}^{\infty}|v|^{3}\left|\int_{0}^{\infty} \Phi(u) V_{u K+1, \delta+i t}\left(n d^{2}\right) e^{i u v} d u\right| d v .
\end{aligned}
$$

4.8. The estimate of $\mathcal{F}_{1}$. As in Lemma 3.6. by Stirling's formula, for $x \asymp K$ we have

$$
\begin{aligned}
V_{x+1, \delta+i t}(y)=\frac{1}{2 \pi i} \int_{\alpha-i \infty}^{\alpha+i \infty} \frac{\widetilde{H}(s+\delta)+\widetilde{H}(s-\delta)}{\left(16 \pi^{2}\right)^{s-\delta}}\left(\frac{x^{2}}{y}\right)^{s-\delta} & \left(1+\frac{2\left(s^{2}-\delta^{2}\right)}{x}\right) d s \\
& +\mathcal{O}\left((1+|t|)^{4} y^{-\varepsilon} K^{-2+\varepsilon}\right)
\end{aligned}
$$

for any $\alpha>|\delta|$. By Lemma 3.6, we can restrict the $c$-sum and $d$-sum to $c d \leq \sqrt{\ell} K^{\varepsilon}$ with a negligible error. (Indeed, since $h$ has compact support, we only need to sum over $n$ which satisfies the condition $\sqrt{\ell n} /(c K) \asymp 1$. So for the sum over $c, d$ with $c d \geq \sqrt{\ell} K^{\varepsilon}$, we have $n d^{2} \asymp(c d K)^{2} / \ell \gg K^{2+2 \varepsilon}$. Note that by Lemma 3.6, we have $V_{4 \pi \sqrt{\ell n} / c+1, \delta+i t}\left(n d^{2}\right) \ll\left(K^{2} /\left(n d^{2}\right)\right)^{B} \ll K^{-B^{\prime}}\left(n d^{2}\right)^{-3}$. Hence this contributes to $\mathcal{F}_{1}$ with $O\left(K^{-B^{\prime \prime}}\right)$ for any $B^{\prime \prime}>0$, here the implied constant depends on both $\varepsilon$ and $B^{\prime \prime}$.) And then by using Weil's bound for the Kloosterman sum, the contribution of the error 
term of $V_{4 \pi \sqrt{\ell n} / c+1, \delta+i t}\left(n d^{2}\right)$ as above to $\mathcal{F}_{1}$ is

$$
\begin{aligned}
& \ll(1+|t|)^{4} K^{-2+\varepsilon} \sum_{d=1}^{\infty} \frac{1}{d^{1+2 \delta}} \sum_{\substack{c \\
c d \ll \sqrt{\ell} K^{\varepsilon}}} \frac{(c, \ell)^{1 / 2}}{c^{1 / 2}} \sum_{n \asymp c^{2} K^{2} / \ell} \frac{\tau(n)}{n^{1 / 2+\delta}} \\
& \ll(1+|t|)^{4} \ell^{-1 / 2} K^{-1+\varepsilon} \sum_{\substack{d \leq \sqrt{\ell} K^{\varepsilon} \\
d^{1+2 \delta}}} \frac{1}{c \ll \sqrt{\ell} K^{\varepsilon}}(c, \ell)^{1 / 2} c^{1 / 2} \\
& \ll(1+|t|)^{4} \ell^{1 / 4} K^{-1+2 \varepsilon} \sum_{c \ll \sqrt{\ell} K^{\varepsilon}}(c, \ell)^{1 / 2} \ll(1+|t|)^{4} \ell^{1 / 4} K^{-1+2 \varepsilon} \sum_{c \ll \sqrt{\ell} K^{\varepsilon}}(c, \ell) \\
& \ll(1+|t|)^{4} \ell^{1 / 4} K^{-1+2 \varepsilon} \sum_{d \mid \ell} d \sum_{\substack{d \mid c \\
c \ll \sqrt{\ell} K^{\varepsilon}}} 1 \ll(1+|t|)^{4} \ell^{1 / 4} K^{-1+3 \varepsilon} .
\end{aligned}
$$

Hence

$$
\begin{aligned}
\mathcal{F}_{1} & =-\frac{\pi}{2} \sum_{c d \leq \sqrt{\ell} K^{\varepsilon}} \frac{1}{c d^{1+2 \delta}} \sum_{\substack{a=1 \\
(a, c)=1}}^{c} e^{\frac{2 \pi i a \ell}{c}} \sum_{n=1}^{\infty} \eta_{i t}(n) e^{\frac{2 \pi i \bar{a} n}{c}} g_{c, d}(n)+\mathcal{O}\left((1+|t|)^{4} \ell^{1 / 4} K^{-1+3 \varepsilon}\right) \\
& =-\frac{\pi}{2} \sum_{d=1}^{\infty} \sum_{c=1}^{\infty} \frac{1}{c d^{1+2 \delta}} \sum_{\substack{a=1 \\
(a, c)=1}}^{c} e^{\frac{2 \pi i a \ell}{c}} \sum_{n=1}^{\infty} \eta_{i t}(n) e^{\frac{2 \pi i \bar{a} n}{c}} g_{c, d}(n)+\mathcal{O}\left((1+|t|)^{4} \ell^{1 / 4} K^{-1+3 \varepsilon}\right),
\end{aligned}
$$

where

$$
\begin{aligned}
g_{c, d}(y):= & \frac{1}{y^{1 / 2+\delta}} \Phi\left(\frac{4 \pi \sqrt{\ell y}}{c K}\right) \\
& \times \frac{1}{2 \pi i} \int_{3-i \infty}^{3+i \infty}[\widetilde{H}(s+\delta)+\widetilde{H}(s-\delta)]\left(\frac{\ell}{c^{2} d^{2}}\right)^{s-\delta}\left(1+\frac{c\left(s^{2}-\delta^{2}\right)}{2 \pi \sqrt{\ell y}}\right) d s .
\end{aligned}
$$

By Lemma 3.12 we have

$$
\mathcal{F}_{1}=\mathcal{M}_{1}+\mathcal{J}_{1}+\mathcal{K}_{1}+\mathcal{O}\left((1+|t|)^{4} \ell^{1 / 4} K^{-1+3 \varepsilon}\right),
$$

where

$$
\begin{aligned}
\mathcal{M}_{1}:= & -\frac{\pi}{2} \zeta(1-2 i t) \sum_{d=1}^{\infty} \sum_{c=1}^{\infty} \frac{S(0, \ell ; c)}{c^{2-2 i t} d^{1+2 \delta}} \int_{0}^{\infty} g_{c, d}(y) y^{-i t} d y \\
& -\frac{\pi}{2} \zeta(1+2 i t) \sum_{d=1}^{\infty} \sum_{c=1}^{\infty} \frac{S(0, \ell ; c)}{c^{2+2 i t} d^{1+2 \delta}} \int_{0}^{\infty} g_{c, d}(y) y^{i t} d y \\
\mathcal{J}_{1}:= & -\frac{\pi}{2} \sum_{d=1}^{\infty} \sum_{c=1}^{\infty} \sum_{n=1}^{\infty} \frac{\eta_{i t}(n) S(0, \ell-n ; c)}{c^{2} d^{1+2 \delta}} \int_{0}^{\infty} g_{c, d}(y) J_{2 i t}^{+}\left(\frac{4 \pi \sqrt{n y}}{c}\right) d y \\
\mathcal{K}_{1}:= & -\frac{\pi}{2} \sum_{d=1}^{\infty} \sum_{c=1}^{\infty} \sum_{n=1}^{\infty} \frac{\eta_{i t}(n) S(0, \ell+n ; c)}{c^{2} d^{1+2 \delta}} \int_{0}^{\infty} g_{c, d}(y) K_{2 i t}^{+}\left(\frac{4 \pi \sqrt{n y}}{c}\right) d y
\end{aligned}
$$

We first deal with $\mathcal{M}_{1}$. Note that

$$
\mathcal{M}_{1}=-\pi \operatorname{Re}\left\{\zeta(1-2 i t) \sum_{d=1}^{\infty} \sum_{c=1}^{\infty} \frac{S(0, \ell ; c)}{c^{2-2 i t} d^{1+2 \delta}} \int_{0}^{\infty} g_{c, d}(y) y^{-i t} d y\right\} .
$$


Introducing the definition of $g_{c, d}(y)$, and making a substitution $u=\frac{4 \pi \sqrt{\ell y}}{c K}$, we obtain

$$
\begin{aligned}
\mathcal{M}_{1}= & -\pi \operatorname{Re}\left\{\zeta(1-2 i t) \sum_{d=1}^{\infty} \sum_{c=1}^{\infty} \frac{S(0, \ell ; c)}{c^{2-2 i t} d^{1+2 \delta}} \int_{0}^{\infty} \frac{1}{y^{1 / 2+\delta+i t}} \Phi\left(\frac{4 \pi \sqrt{\ell y}}{c K}\right)\right. \\
& \left.\times \frac{1}{2 \pi i} \int_{3-i \infty}^{3+i \infty}[\widetilde{H}(s+\delta)+\widetilde{H}(s-\delta)]\left(\frac{\ell}{c^{2} d^{2}}\right)^{s-\delta}\left(1+\frac{c\left(s^{2}-\delta^{2}\right)}{2 \pi \sqrt{\ell y}}\right) d s d y\right\} \\
= & -\pi \operatorname{Re}\left\{\zeta(1-2 i t) \frac{2 K^{1-2 \delta-2 i t}}{(4 \pi)^{1-2 \delta-2 i t} \ell^{1 / 2-\delta-i t}}\right. \\
& \times \int_{0}^{\infty} \Phi(u) u^{-2 \delta-2 i t} \frac{1}{2 \pi i} \int_{3-i \infty}^{3+i \infty}[\widetilde{H}(s+\delta)+\widetilde{H}(s-\delta)] \ell^{s-\delta}\left(1+\frac{2\left(s^{2}-\delta^{2}\right)}{K u}\right) \\
& \left.\times \sum_{d=1}^{\infty} \sum_{c=1}^{\infty} \frac{S(0, \ell ; c)}{c^{1+2 s} d^{1+2 s}} d s d u\right\} .
\end{aligned}
$$

Note that for $\operatorname{Re}(s)>0$ and $\ell \geq 1$ we have

$$
\sum_{d=1}^{\infty} \sum_{c=1}^{\infty} \frac{S(0, \ell ; c)}{c^{1+2 s} d^{1+2 s}}=\ell^{-s} \eta_{s}(\ell)
$$

Hence we get

$$
\begin{aligned}
\mathcal{M}_{1}= & -\pi \operatorname{Re}\left\{\zeta(1-2 i t) \frac{2 K^{1-2 \delta-2 i t}}{(4 \pi)^{1-2 \delta-2 i t} \ell^{1 / 2-i t}} \int_{0}^{\infty} \Phi(u) u^{-2 \delta-2 i t}\right. \\
& \left.\times \frac{1}{2 \pi i} \int_{3-i \infty}^{3+i \infty}[\widetilde{H}(s+\delta)+\widetilde{H}(s-\delta)] \eta_{s}(\ell)\left(1+\frac{2\left(s^{2}-\delta^{2}\right)}{K u}\right) d s d u\right\} .
\end{aligned}
$$

For the innermost integral, we change $s$ to $-s$. Recall that $\widetilde{H}(-s)=-\widetilde{H}(s)$ and $\eta_{s}(\ell)=$ $\eta_{-s}(\ell)$. So that for any $u \asymp 1$ we have

$$
\begin{aligned}
& \frac{1}{2 \pi i} \int_{3-i \infty}^{3+i \infty}[\widetilde{H}(s+\delta)+\widetilde{H}(s-\delta)] \eta_{s}(\ell)\left(1+\frac{2\left(s^{2}-\delta^{2}\right)}{K u}\right) d s \\
& \quad=-\frac{1}{2 \pi i} \int_{-3+i \infty}^{-3-i \infty}[\widetilde{H}(-s+\delta)+\widetilde{H}(-s-\delta)] \eta_{-s}(\ell)\left(1+\frac{2\left(s^{2}-\delta^{2}\right)}{K u}\right) d s \\
& \quad=-\frac{1}{2 \pi i} \int_{-3-i \infty}^{-3+i \infty}[\widetilde{H}(s+\delta)+\widetilde{H}(s-\delta)] \eta_{s}(\ell)\left(1+\frac{2\left(s^{2}-\delta^{2}\right)}{K u}\right) d s \\
& \quad=\frac{1}{2} \frac{1}{2 \pi i}\left(\int_{3-i \infty}^{3+i \infty}-\int_{-3-i \infty}^{-3+i \infty}\right)[\widetilde{H}(s+\delta)+\widetilde{H}(s-\delta)] \eta_{s}(\ell)\left(1+\frac{2\left(s^{2}-\delta^{2}\right)}{K u}\right) d s \\
& =\eta_{\delta}(\ell) .
\end{aligned}
$$

Therefore, we get

$$
\mathcal{M}_{1}=-\frac{1}{2} \operatorname{Re}\left\{\zeta(1-2 i t) \eta_{\delta}(\ell) \frac{K^{1-2 \delta-2 i t}}{(4 \pi)^{-2 \delta-2 i t} \ell^{1 / 2-i t}} \int_{0}^{\infty} \Phi(u) u^{-2 \delta-2 i t} d u\right\} .
$$


To handle the $\mathcal{J}_{1}$ and $\mathcal{K}_{1}$ terms, we need some asymptotic evaluations regarding the Bessel functions (cf. Hough [9, Eq. (3.5) and (3.6)])

$$
\begin{aligned}
J_{\nu}^{+}(x)= & -\sqrt{\frac{2 \pi}{x}} \sin (x-\pi / 4)\left[1-\frac{16 \nu^{4}-40 \nu^{2}+9}{128 x^{2}}\right] \\
& -\pi \cos (x-\pi / 4) \frac{\nu^{2}-1 / 4}{2 x}+\mathcal{O}\left(\frac{1+|\nu|^{6}}{x^{3}}\right), \\
K_{\nu}(x)= & \sqrt{\frac{\pi}{2 x}} e^{-x}\left[1+\mathcal{O}\left(\frac{1+|\nu|^{2}}{x}\right)\right] .
\end{aligned}
$$

Substituting $u=\frac{4 \pi \sqrt{\ell y}}{c K}$ we obtain

$$
\begin{aligned}
\mathcal{J}_{1}= & -\pi\left(\frac{K}{4 \pi}\right)^{1-2 \delta} \frac{1}{\ell^{1 / 2-\delta}} \sum_{d=1}^{\infty} \sum_{c=1}^{\infty} \sum_{n=1}^{\infty} \frac{\eta_{i t}(n) S(0, \ell-n ; c)}{c^{1+2 \delta} d^{1+2 \delta}} \int_{0}^{\infty} \Phi(u) u^{-2 \delta} J_{2 i t}^{+}\left(\frac{\sqrt{n} K u}{\sqrt{\ell}}\right) \\
& \times \frac{1}{2 \pi i} \int_{3-i \infty}^{3+i \infty}[\widetilde{H}(s+\delta)+\widetilde{H}(s-\delta)]\left(\frac{\ell}{c^{2} d^{2}}\right)^{s-\delta}\left(1+\frac{2\left(s^{2}-\delta^{2}\right)}{K u}\right) d s d u, \\
\mathcal{K}_{1}= & -\pi\left(\frac{K}{4 \pi}\right)^{1-2 \delta} \frac{1}{\ell^{1 / 2-\delta}} \sum_{d=1}^{\infty} \sum_{c=1}^{\infty} \sum_{n=1}^{\infty} \frac{\eta_{i t}(n) S(0, \ell+n ; c)}{c^{1+2 \delta} d^{1+2 \delta}} \int_{0}^{\infty} \Phi(u) u^{-2 \delta} K_{2 i t}^{+}\left(\frac{\sqrt{n} K u}{\sqrt{\ell}}\right) \\
& \times \frac{1}{2 \pi i} \int_{3-i \infty}^{3+i \infty}[\widetilde{H}(s+\delta)+\widetilde{H}(s-\delta)]\left(\frac{\ell}{c^{2} d^{2}}\right)^{s-\delta}\left(1+\frac{2\left(s^{2}-\delta^{2}\right)}{K u}\right) d s d u .
\end{aligned}
$$

Since $S(0,0 ; c)=\phi(c)$ where $\phi$ is the Euler function, and

$$
\sum_{d=1}^{\infty} \sum_{c=1}^{\infty} \frac{\phi(c)}{c^{1+2 s} d^{1+2 s}}=\zeta(2 s) \quad(\text { for } \operatorname{Re}(s)>1 / 2),
$$

together with (4.10), we have

$$
\mathcal{J}_{1}=\mathcal{J}_{11}+\mathcal{J}_{12},
$$

and

$$
\begin{aligned}
& \mathcal{K}_{1}=-\pi\left(\frac{K}{4 \pi}\right)^{1-2 \delta} \frac{1}{\ell^{1 / 2-\delta}} \sum_{n=1}^{\infty} \eta_{i t}(n) \int_{0}^{\infty} \Phi(u) u^{-2 \delta} K_{2 i t}^{+}\left(\frac{\sqrt{n} K u}{\sqrt{\ell}}\right) \\
& \times \frac{1}{2 \pi i} \int_{3-i \infty}^{3+i \infty}[\widetilde{H}(s+\delta)+\widetilde{H}(s-\delta)] \frac{\ell^{s-\delta}}{(\ell+n)^{s}} \eta_{s}(\ell+n)\left(1+\frac{2\left(s^{2}-\delta^{2}\right)}{K u}\right) d s d u,
\end{aligned}
$$

where

$$
\begin{aligned}
\mathcal{J}_{11}:= & -\pi\left(\frac{K}{4 \pi}\right)^{1-2 \delta} \frac{1}{\ell^{1 / 2-\delta}} \sum_{n \neq \ell} \eta_{i t}(n) \int_{0}^{\infty} \Phi(u) u^{-2 \delta} J_{2 i t}^{+}\left(\frac{\sqrt{n} K u}{\sqrt{\ell}}\right) \\
& \times \frac{1}{2 \pi i} \int_{3-i \infty}^{3+i \infty}[\widetilde{H}(s+\delta)+\widetilde{H}(s-\delta)] \ell^{s-\delta} \sum_{a \mid(\ell-n)} \frac{1}{a^{2 s}}\left(1+\frac{2\left(s^{2}-\delta^{2}\right)}{K u}\right) d s d u \\
\mathcal{J}_{12}:= & -\pi\left(\frac{K}{4 \pi}\right)^{1-2 \delta} \frac{\eta_{i t}(\ell)}{\ell^{1 / 2-\delta}} \int_{0}^{\infty} \Phi(u) u^{-2 \delta} J_{2 i t}^{+}(K u) \\
& \times \frac{1}{2 \pi i} \int_{3-i \infty}^{3+i \infty}[\widetilde{H}(s+\delta)+\widetilde{H}(s-\delta)] \ell^{s-\delta} \zeta(2 s)\left(1+\frac{2\left(s^{2}-\delta^{2}\right)}{K u}\right) d s d u
\end{aligned}
$$


Note that $\ell \leq K^{2-2 \vartheta}$ and the $K$-Bessel function is exponentially small for large variable. It follows that $\mathcal{K}_{1}$ is extremely small.

Now we consider $\mathcal{J}_{11}$. By shifting the contour of the integral in $\mathcal{J}_{11}$ to $\operatorname{Re}(s)=\delta+\varepsilon$, the contribution coming from the error of the asymptotic expansion of $J_{2 i t}^{+}\left(\frac{\sqrt{n} K u}{\sqrt{\ell}}\right)$ is

$$
\begin{aligned}
& \ll(1+|t|)^{6}\left(\frac{K}{\sqrt{\ell}}\right)^{1-2 \delta} \sum_{n \neq \ell} \tau(n) \int_{0}^{\infty} \Phi(u) u^{-2 \delta}\left(\frac{\sqrt{n} K u}{\sqrt{\ell}}\right)^{-3} \\
& \times\left|\frac{1}{2 \pi i} \int_{\delta+\varepsilon-i \infty}^{\delta+\varepsilon+i \infty}[\widetilde{H}(s+\delta)+\widetilde{H}(s-\delta)] \ell^{s-\delta} \sum_{a \mid(\ell-n)} \frac{1}{a^{2 s}}\left(1+\frac{2\left(s^{2}-\delta^{2}\right)}{K u}\right) d s\right| d u \\
& \ll(1+|t|)^{6} \ell K^{-2+\varepsilon} .
\end{aligned}
$$

And similarly, by shifting the contour of the integral in $\mathcal{J}_{12}$ to $\operatorname{Re}(s)=1 / 2+\varepsilon$, the contribution coming from the error of the asymptotic expansion of $J_{2 i t}^{+}(K u)$ is $\ll(1+|t|)^{6} \ell^{-1 / 2} K^{-2+\varepsilon}$. We only show how to bound the contribution from the main term

$$
-\left(\frac{2 \pi}{K u}\right)^{1 / 2}\left(\frac{\ell}{n}\right)^{1 / 4} \sin \left(K u\left(\frac{n}{\ell}\right)^{1 / 2}-\frac{\pi}{4}\right)
$$

to $\mathcal{J}_{11}$; the rest of the main terms can be handled in the same way, and their contribution is smaller. The contribution to $\mathcal{J}_{11}$ from integrating against 4.16 is

$$
\begin{aligned}
\mathcal{J}_{111}:= & \pi\left(\frac{K}{4 \pi}\right)^{1-2 \delta} \frac{1}{\ell^{1 / 2-\delta}}\left(\frac{2 \pi}{K}\right)^{1 / 2} \ell^{1 / 4} \\
& \times \sum_{n \neq \ell} \frac{\eta_{i t}(n)}{n^{1 / 4}} \int_{0}^{\infty} \Phi(u) u^{-1 / 2-2 \delta} \sin \left(K\left(\frac{n}{\ell}\right)^{1 / 2} u-\frac{\pi}{4}\right) \\
& \times \frac{1}{2 \pi i} \int_{3-i \infty}^{3+i \infty}[\tilde{H}(s+\delta)+\widetilde{H}(s-\delta)] \ell^{s-\delta} \sum_{a \mid(\ell-n)} \frac{1}{a^{2 s}}\left(1+\frac{2\left(s^{2}-\delta^{2}\right)}{K u}\right) d s d u
\end{aligned}
$$

Define

$$
g_{n}(u):=\frac{1}{2 \pi i} \int_{3-i \infty}^{3+i \infty}[\widetilde{H}(s+\delta)+\widetilde{H}(s-\delta)] \ell^{s-\delta} \sum_{a \mid(\ell-n)} \frac{1}{a^{2 s}}\left(1+\frac{2\left(s^{2}-\delta^{2}\right)}{K u}\right) d s .
$$

Then by integrating by parts twice, we obtain

$$
\begin{aligned}
\mathcal{J}_{111} & \ll\left(\frac{K}{\sqrt{\ell}}\right)^{1 / 2-2 \delta} \sum_{n \neq \ell} \frac{\tau(n)}{n^{1 / 4}}\left|\int_{0}^{\infty} \Phi(u) u^{-1 / 2-2 \delta} g_{n}(u) \sin \left(K\left(\frac{n}{\ell}\right)^{1 / 2} u-\frac{\pi}{4}\right) d u\right| \\
& \ll\left(\frac{K}{\sqrt{\ell}}\right)^{-3 / 2} \sum_{n \neq \ell} \frac{\tau(n)}{n^{5 / 4}}\left|\int_{0}^{\infty}\left(\Phi(u) u^{-1 / 2-2 \delta} g_{n}(u)\right)^{\prime \prime} \sin \left(K\left(\frac{n}{\ell}\right)^{1 / 2} u-\frac{\pi}{4}\right) d u\right| .
\end{aligned}
$$

By shifting the contour of the integral in $g_{n}(u)$ to the line $\operatorname{Re}(s)=\delta+\varepsilon$, and then bounding everything trivially, we get

$$
\mathcal{J}_{111} \ll \ell^{3 / 4} K^{-3 / 2+\varepsilon} .
$$


Hence, by 4.9, 4.11), 4.14, 4.15), and (4.17), we obtain

$$
\begin{aligned}
\mathcal{F}_{1}=-\frac{1}{2} \operatorname{Re}\{ & \left.\zeta(1-2 i t) \eta_{\delta}(\ell) \frac{K^{1-2 \delta-2 i t}}{(4 \pi)^{-2 \delta-2 i t} \ell^{1 / 2-i t}} \int_{0}^{\infty} \Phi(u) u^{-2 \delta-2 i t} d u\right\} \\
& +\mathcal{O}\left((1+|t|)^{4} \ell^{1 / 4} K^{-1+3 \varepsilon}+\ell^{3 / 4} K^{-3 / 2+\varepsilon}+(1+|t|)^{6} \ell K^{-2+\varepsilon}\right) .
\end{aligned}
$$

4.19. The treatment of $\mathcal{F}_{2}$ and $\mathcal{E}$. Define

$$
\mathcal{W}_{K}(y, v)=\mathcal{W}_{K}(y, v, \Phi):=\int_{0}^{\infty} \frac{\Phi(\sqrt{u})}{\sqrt{2 \pi u}} V_{\sqrt{u} K+1, \delta+i t}(y) e^{i u v} d u,
$$

for $y \geq 1$ and $0<v<K^{5}$. Using the integral definition of $V_{k, \delta+i t}$ we have

$$
\begin{aligned}
\mathcal{W}_{K}(y, v)= & \frac{1}{2 \pi i} \int_{\alpha-i \infty}^{\alpha+i \infty} \frac{\tilde{H}(s+\delta)+\widetilde{H}(s-\delta)}{\left(4 \pi^{2} y\right)^{s-\delta}} \\
& \times \int_{0}^{\infty} \frac{\Gamma\left(s+\frac{\sqrt{u} K+1}{2}+i t\right) \Gamma\left(s+\frac{\sqrt{u} K+1}{2}-i t\right)}{\Gamma\left(\delta+\frac{\sqrt{u} K+1}{2}+i t\right) \Gamma\left(\delta+\frac{\sqrt{u} K+1}{2}-i t\right)} \frac{\Phi(\sqrt{u})}{\sqrt{2 \pi u}} e^{i u v} d u d s,
\end{aligned}
$$

for any $\alpha>|\delta|$. Taking $\alpha=A+\delta$ for any large number $A>1$. For $|\operatorname{Im}(s)| \geq K^{\varepsilon}$, then by the properties of $\widetilde{H}$ we have $\mathcal{W}_{K}(y, v) \ll\left(\frac{K^{2}}{y}\right)^{A} K^{-B}$ for any $B>0$. And for $|\operatorname{Im}(s)| \leq K^{\varepsilon}$, by integrating by parts several times and asymptotic formulas for Gamma function and the polygamma functions, we have

$$
\int_{0}^{\infty} \frac{\Gamma\left(s+\frac{\sqrt{u} K+1}{2}+i t\right) \Gamma\left(s+\frac{\sqrt{u} K+1}{2}-i t\right)}{\Gamma\left(\delta+\frac{\sqrt{u} K+1}{2}+i t\right) \Gamma\left(\delta+\frac{\sqrt{u} K+1}{2}-i t\right)} \frac{\Phi(\sqrt{u})}{\sqrt{2 \pi u}} e^{i u v} d u \ll_{A, B} \frac{1+|s|}{v^{B}} K^{2 A} .
$$

Hence we obtain

$$
\mathcal{W}_{K}(y, v) \ll_{A, B}\left(\frac{K^{2}}{y}\right)^{A} v^{-B} .
$$

Thus we can truncate the sum over $n$ in $\mathcal{F}_{2}$ at $n d^{2} \leq K^{2+\varepsilon}$ with a negligible error. Note that $\ell \leq K^{2-2 \vartheta}$, so we have $\frac{c K^{2}}{8 \pi \sqrt{\ell n}} \gg K^{\vartheta / 2}$. Hence by 4.20 , for any $B>0$ we get

$$
\mathcal{F}_{2} \ll_{B} K^{-B} \text {. }
$$

Similarly, we can show that

$$
\int_{-\infty}^{\infty}|v|^{3}\left|\int_{0}^{\infty} \Phi(u) V_{u K+1, \delta+i t}(y) e^{i u v} d u\right| d v \ll_{A}\left(\frac{K^{2}}{y}\right)^{A} .
$$

So we can truncate the sum over $n$ and $d$ in $\mathcal{E}$ at $n d^{2} \leq K^{2+\varepsilon}$ with a negligible error. Hence we get

$$
\mathcal{E} \ll \frac{\ell^{1 / 2}}{K^{3}} \sum_{d=1}^{\infty} \sum_{n d^{2} \leq K^{2+\varepsilon}}^{\infty} \frac{\tau(n)}{d^{1+2 \delta} n^{\delta}} \sum_{\substack{c \\ c d \leq \sqrt{\ell} K^{1+\varepsilon}}} \frac{|S(n, \ell ; c)|}{c^{2}}+\mathcal{O}\left(K^{-B}\right) \ll \ell^{1 / 2} K^{-1+2 \varepsilon} .
$$

Combining (4.7), 4.18, 4.21, and 4.22, we have

$$
\begin{aligned}
\mathcal{F}= & -\frac{1}{2} \operatorname{Re}\left\{\zeta(1-2 i t) \eta_{\delta}(\ell) \frac{K^{1-2 \delta-2 i t}}{(4 \pi)^{-2 \delta-2 i t} \ell^{1 / 2-i t}} \int_{0}^{\infty} \Phi(u) u^{-2 \delta-2 i t} d u\right\} \\
& +\mathcal{O}\left((1+|t|)^{4} \ell^{1 / 2} K^{-1+\varepsilon}\right) .
\end{aligned}
$$

Using 4.3, 4.5, and 4.23, this completes the proof of Theorem 4.2 


\section{Mollification near the critical point}

5.1. Choosing the mollifier. We will take the same mollifier as in Hough [9, §5]. Let

$$
L(s, f)^{-1}:=\sum_{n=1}^{\infty} \frac{a_{f}(n)}{n^{s}} \quad(\operatorname{Re}(s)>1) .
$$

The coefficients $a_{f}(n)$ are supported on cube-free numbers. Now, for $m, n$ square-free, $(m, n)=1$, we have $a_{f}\left(m n^{2}\right)=\mu(m) \lambda_{f}(m)$.

Fix $0<\Upsilon<1$ and $M \leq K^{1-2 \vartheta}$. Define

$$
F_{\Upsilon, M}(x):= \begin{cases}1, & \text { if } 0 \leq x \leq M^{1-\Upsilon}, \\ P\left(\frac{\log (M / x)}{\log M}\right), & \text { if } M^{1-\Upsilon} \leq x \leq M, \\ 0, & \text { if } x \geq M,\end{cases}
$$

with $P(t)$, a degree 3 polynomial, that satisfies $P(\Upsilon)=1$ and $P^{\prime}(\Upsilon)=P(0)=P^{\prime}(0)=0$.

We define the mollifier for $L(s, f)$ by

$$
M(s, f):=\sum_{n=1}^{\infty} \frac{a_{f}(n) F_{\Upsilon, M}(\operatorname{rad}(n))}{n^{s}}=\sum_{\ell=1}^{\infty} \frac{x_{\ell}(s)}{\ell^{s}} \lambda_{f}(\ell),
$$

where $\operatorname{rad}(n)$ denotes the product of the distinct prime numbers dividing $n$ and

$$
x_{\ell}(s):=\sum_{n=1}^{\infty} \frac{\mu^{2}(\ell n) \mu(\ell) F_{\Upsilon, M}(\ell n)}{n^{2 s}} .
$$

We now set $\omega:=\delta+i t$. By 3.8 , we have

$$
\begin{aligned}
|M(1 / 2+\omega, f)|^{2} & =\sum_{\ell_{1}=1}^{\infty} \sum_{\ell_{2}=1}^{\infty} \frac{x_{\ell_{1}}(1 / 2+\omega) x_{\ell_{2}}(1 / 2+\bar{\omega})}{\ell_{1}^{1 / 2+\omega} \ell_{2}^{1 / 2+\bar{\omega}}} \lambda_{f}\left(\ell_{1}\right) \lambda_{f}\left(\ell_{2}\right) \\
& =\sum_{\ell_{1}=1}^{\infty} \sum_{\ell_{2}=1}^{\infty} \sum_{d=1}^{\infty} \frac{1}{d^{1+\omega+\bar{\omega}}} \frac{x_{d \ell_{1}}(1 / 2+\omega) x_{d \ell_{2}}(1 / 2+\bar{\omega})}{\ell_{1}^{1 / 2+\omega} \ell_{2}^{1 / 2+\bar{\omega}}} \lambda_{f}\left(\ell_{1} \ell_{2}\right) .
\end{aligned}
$$

We may always write $L(s, f) M(s, f)$ as $L M(s, f)$. Hence we get

$$
\begin{aligned}
\mathcal{A}\left(\left\{|L M(1 / 2+\omega, f)|^{2}\right\} ; K, \Phi\right)= & \sum_{\ell_{1}=1}^{\infty} \sum_{\ell_{2}=1}^{\infty} \sum_{d=1}^{\infty} \frac{1}{d^{1+\omega+\bar{\omega}}} \frac{x_{d \ell_{1}}(1 / 2+\omega) x_{d \ell_{2}}(1 / 2+\bar{\omega})}{\ell_{1}^{1 / 2+\omega} \ell_{2}^{1 / 2+\bar{\omega}}} \\
& \times \mathcal{A}\left(\left\{\lambda_{f}\left(\ell_{1} \ell_{2}\right)|L(1 / 2+\omega, f)|^{2}\right\} ; K, \Phi\right) .
\end{aligned}
$$

There are three cases we need to consider when $\omega=\delta+i t$ :

$$
\begin{gathered}
(\mathbf{I})\left\{\begin{array}{l}
\frac{-B}{\log K} \leq \delta \leq \frac{C \log \log K}{\log K} \quad \text { and } \quad|\delta| \geq \frac{C}{\log K \log \log K}, \\
\frac{C}{\log K \log \log K} \leq|t| \leq \frac{C \log \log K}{\log K} ;
\end{array}\right. \\
(\text { II })\left\{\begin{array} { l } 
{ | \delta | \leq \frac { C } { \operatorname { l o g } K \operatorname { l o g } \operatorname { l o g } K } , } \\
{ \frac { C _ { 1 } } { \operatorname { l o g } K } \leq | t | \leq \frac { C _ { 2 } } { \operatorname { l o g } K } ; }
\end{array} \quad ( \text { III } ) \left\{\begin{array}{l}
\frac{A}{\log K} \leq|\delta| \leq \frac{C \log \log K}{\log K}, \\
|t| \leq \frac{C}{\log K \log \log K},
\end{array}\right.\right.
\end{gathered}
$$


where $A, B$, and $C$ are some constants. We will focus on the first case $(\mathbf{I})$, which we will assume in this section from now on. Note that the other cases can be handled by combining the method of Conrey-Soundararajan [2, §6]. Define

$$
\nu_{(\alpha, \beta)}(\ell):=\frac{\eta_{(\alpha-\beta) / 2}(\ell)}{\ell^{(\alpha+\beta) / 2}},
$$

and

$$
\varepsilon(\alpha, \beta):= \begin{cases}1, & \text { if }(\alpha, \beta)=(\omega, \bar{\omega}), \\ -\left(\frac{K}{4 \pi}\right)^{-2 \omega}, & \text { if }(\alpha, \beta)=(-\omega, \bar{\omega}), \\ -\left(\frac{K}{4 \pi}\right)^{-2 \bar{\omega}}, & \text { if }(\alpha, \beta)=(\omega,-\bar{\omega}), \\ \left(\frac{K}{4 \pi}\right)^{-2 \omega-2 \bar{\omega}}, & \text { if }(\alpha, \beta)=(-\omega,-\bar{\omega}) .\end{cases}
$$

By Theorem 4.2 we obtain

$$
\begin{aligned}
& \mathcal{A}\left(\left\{|L M(1 / 2+\omega, f)|^{2}\right\} ; K, \Phi\right) \\
= & \sum_{\ell_{1}=1}^{\infty} \sum_{\ell_{2}=1}^{\infty} \sum_{d=1}^{\infty} \frac{1}{d^{1+\omega+\bar{\omega}}} \frac{x_{d \ell_{1}}(1 / 2+\omega) x_{d \ell_{2}}(1 / 2+\bar{\omega})}{\ell_{1}^{1 / 2+\omega} \ell_{2}^{1 / 2+\bar{\omega}}} \\
& \times\left[\zeta(1+2 \delta) \frac{\eta_{i t}\left(\ell_{1} \ell_{2}\right)}{\left(\ell_{1} \ell_{2}\right)^{1 / 2+\delta}} \frac{K}{4} \int_{0}^{\infty} \Phi(u) d u\right. \\
& +\zeta(1-2 \delta) \frac{\eta_{i t}\left(\ell_{1} \ell_{2}\right)}{\left(\ell_{1} \ell_{2}\right)^{1 / 2-\delta}}\left(\frac{K}{4 \pi}\right)^{-4 \delta} \frac{K}{4} \int_{0}^{\infty} \Phi(u) u^{-4 \delta} d u \\
& \left.-2 \operatorname{Re}\left\{\zeta(1-2 i t) \frac{\eta_{\delta}\left(\ell_{1} \ell_{2}\right)}{\left(\ell_{1} \ell_{2}\right)^{1 / 2-i t}}\left(\frac{K}{4 \pi}\right)^{-2 \delta-2 i t} \frac{K}{4} \int_{0}^{\infty} \Phi(u) u^{-2 \delta-2 i t} d u\right\}\right]+\mathcal{O}\left(M K^{\varepsilon}\right) \\
= & \sum_{(\alpha, \beta)=( \pm \omega, \pm \bar{\omega})} \Psi(\alpha, \beta) \mathcal{V}_{(\alpha, \beta)}(\omega)+\mathcal{O}\left(M K^{\varepsilon}\right),
\end{aligned}
$$

where for any $(\alpha, \beta)=( \pm \omega, \pm \bar{\omega})$,

$$
\Psi(\alpha, \beta):=\frac{K}{4} \zeta(1+\alpha+\beta) \widetilde{\Phi}(1-\omega-\bar{\omega}+\alpha+\beta) \varepsilon(\alpha, \beta),
$$

and

$$
\mathcal{V}_{(\alpha, \beta)}(\omega):=\sum_{\ell=1}^{\infty} \nu_{(\alpha, \beta)}(\ell) \sum_{\ell_{1} \ell_{2}=\ell} \sum_{d=1}^{\infty} \frac{1}{d^{1+\omega+\bar{\omega}}} \frac{x_{d \ell_{1}}(1 / 2+\omega) x_{d \ell_{2}}(1 / 2+\bar{\omega})}{\ell_{1}^{1+\omega} \ell_{2}^{1+\bar{\omega}}}
$$

By the multiplicative property of the divisor function

$$
\eta_{\nu}(m n)=\sum_{d \mid(m, n)} \mu(d) \eta_{\nu}(m / d) \eta_{\nu}(n / d)
$$

we can now obtain the following lemma.

5.10. Lemma. Let $\omega \in \mathbb{C}$, and let $(\alpha, \beta)=( \pm \omega, \pm \bar{\omega})$. We have

$$
\mathcal{V}_{(\alpha, \beta)}(\omega)=\sum_{r=1}^{\infty} \frac{1}{r^{1+\omega+\bar{\omega}}} \tau_{(\alpha, \beta)}(r) S_{(\alpha, \beta)}(r ; \omega) S_{(\alpha, \beta)}(r ; \bar{\omega}),
$$

where, for $z \in\{\omega, \bar{\omega}\}$,

$$
\tau_{(\alpha, \beta)}(r):=\sum_{d \mid r} \frac{\mu(d)}{d^{1+\alpha+\beta}}, \quad S_{(\alpha, \beta)}(r ; z):=\sum_{\ell=1}^{\infty} \frac{\nu_{(\alpha, \beta)}(\ell) x_{\ell r}(1 / 2+z)}{\ell^{1+z}} .
$$


Proof. By 5.9 we have

$$
\begin{aligned}
& \mathcal{V}_{(\alpha, \beta)}(\omega) \\
& =\sum_{\ell_{1}=1}^{\infty} \sum_{\ell_{2}=1}^{\infty} \sum_{d=1}^{\infty} \sum_{a \mid\left(\ell_{1}, \ell_{2}\right)} \frac{\mu(a)}{d^{1+\omega+\bar{\omega}}} \frac{\eta_{(\alpha-\beta) / 2}\left(\ell_{1} / a\right) \eta_{(\alpha-\beta) / 2}\left(\ell_{2} / a\right)}{\left(\ell_{1} \ell_{2}\right)^{(\alpha+\beta) / 2}} \frac{x_{d \ell_{1}}(1 / 2+\omega) x_{d \ell_{2}}(1 / 2+\bar{\omega})}{\ell_{1}^{1+\omega} \ell_{2}^{1+\bar{\omega}}} \\
& =\sum_{\ell_{1}=1}^{\infty} \sum_{\ell_{2}=1}^{\infty} \sum_{d=1}^{\infty} \sum_{a \mid \ell_{1}} \sum_{a \mid \ell_{2}} \frac{\mu(a)}{d^{1+\omega+\bar{\omega}} a^{\alpha+\beta}} \nu_{(\alpha, \beta)}\left(\ell_{1} / a\right) \nu_{(\alpha, \beta)}\left(\ell_{2} / a\right) \frac{x_{d \ell_{1}}(1 / 2+\omega) x_{d \ell_{2}}(1 / 2+\bar{\omega})}{\ell_{1}^{1+\omega} \ell_{2}^{1+\bar{\omega}}} \\
& =\sum_{d=1}^{\infty} \sum_{a=1}^{\infty} \sum_{\ell_{1}=1}^{\infty} \sum_{\ell_{2}=1}^{\infty} \frac{\mu(a)}{d^{1+\omega+\bar{\omega}} a^{2+\omega+\bar{\omega}+\alpha+\beta}} \nu_{(\alpha, \beta)}\left(\ell_{1}\right) \nu_{(\alpha, \beta)}\left(\ell_{2}\right) \frac{x_{a d \ell_{1}}(1 / 2+\omega) x_{a d \ell_{2}}(1 / 2+\bar{\omega})}{\ell_{1}^{1+\omega} \ell_{2}^{1+\bar{\omega}}} \\
& =\sum_{r=1}^{\infty} \frac{1}{r^{1+\omega+\bar{\omega}}} \sum_{a \mid r} \frac{\mu(a)}{a^{1+\alpha+\beta}} S_{(\alpha, \beta)}(r ; \omega) S_{(\alpha, \beta)}(r ; \bar{\omega}) .
\end{aligned}
$$

This completes the proof of the lemma.

For any $(\alpha, \beta)=( \pm \omega, \pm \bar{\omega})$, we define

$$
\begin{aligned}
& \mathcal{V}_{(\alpha, \beta)}^{\leq}(\omega):=\sum_{1 \leq r \leq M^{1-\Upsilon}} \frac{1}{r^{1+\omega+\bar{\omega}}} \tau_{(\alpha, \beta)}(r) S_{(\alpha, \beta)}(r ; \omega) S_{(\alpha, \beta)}(r ; \bar{\omega}), \\
& \mathcal{V}_{(\alpha, \beta)}^{>}(\omega):=\sum_{M^{1-\Upsilon<r \leq M}} \frac{1}{r^{1+\omega+\bar{\omega}}} \tau_{(\alpha, \beta)}(r) S_{(\alpha, \beta)}(r ; \omega) S_{(\alpha, \beta)}(r ; \bar{\omega}),
\end{aligned}
$$

and we refer to these as the summation of the short-range and long-range terms, respectively. Note that $S_{(\alpha, \beta)}(r ; z)=0$ if $r>M$, for $z \in\{\omega, \bar{\omega}\}$.

5.13. Treatment of $S_{(\alpha, \beta)}(r ; z)$. For $z \in\{\omega, \bar{\omega}\}$, any integer $r \geq 1$, any real $y>0$, any complex number $s \in \mathbb{C}$ with $\operatorname{Re}(s)>3|\delta|$, and any polynomial $R$, we define

$$
\begin{aligned}
T_{(\alpha, \beta)}(s ; r ; z) & :=\sum_{\ell \geq 1} \frac{\nu_{(\alpha, \beta)}(\ell)}{\ell^{1+s+z}} \sum_{n \geq 1} \frac{\mu^{2}(\ell n r) \mu(\ell r)}{n^{1+s+2 z}}, \\
T_{(\alpha, \beta)}(y, R ; r ; z) & :=\sum_{\ell \geq 1} \frac{\nu_{(\alpha, \beta)}(\ell)}{\ell^{1+z}} \sum_{1 \leq n \leq y /(\ell r)} \frac{\mu^{2}(\ell n r) \mu(\ell r)}{n^{1+2 z}} R\left(\frac{\log (y /(\ell n r))}{\log y}\right) .
\end{aligned}
$$

Let

$$
Q(x):=1-P(\Upsilon+(1-\Upsilon) x)
$$

We remark that

$$
\begin{array}{ll}
S_{(\alpha, \beta)}(r ; z)=T_{(\alpha, \beta)}(M, P ; r ; z)+T_{(\alpha, \beta)}\left(M^{1-\Upsilon}, Q ; r ; z\right), & \text { if } r \leq M^{1-\Upsilon} \\
S_{(\alpha, \beta)}(r ; z)=T_{(\alpha, \beta)}(M, P ; r ; z), & \text { if } M^{1-\Upsilon}<r \leq M .
\end{array}
$$

5.19. Lemma. Let $z \in\{\omega, \bar{\omega}\}$ and $(\alpha, \beta)=( \pm \omega, \pm \bar{\omega})$. For $\operatorname{Re}(s)>-\delta+|\delta|$, we have

$$
T_{(\alpha, \beta)}(s ; r ; z)=\frac{\mu(r) G_{(\alpha, \beta)}(s ; r ; z) \zeta(1+s+2 z)}{\zeta(1+s+z+\alpha) \zeta(1+s+z+\beta)},
$$


where $G_{(\alpha, \beta)}(s ; r ; z):=\prod_{p} G_{p,(\alpha, \beta)}(s ; r ; z)$ with

$$
G_{p,(\alpha, \beta)}(s ; r ; z):=\left\{\begin{array}{cl}
\left(1-p^{-1-s-z-\alpha}\right)^{-1}\left(1-p^{-1-s-z-\beta}\right)^{-1}\left(1-p^{-1-s-2 z}\right), & \text { if } p \mid r, \\
\left(1-p^{-1-s-z-\alpha}\right)^{-1}\left(1-p^{-1-s-z-\beta}\right)^{-1}\left(1-p^{-1-s-2 z}\right) & \\
\times\left(1-p^{-1-s-z-\alpha}-p^{-1-s-z-\beta}+p^{-1-s-2 z}\right), & \text { if } p \nmid r,
\end{array}\right.
$$

so that $G_{(\alpha, \beta)}(s ; r ; z)$ is holomorphic in $\operatorname{Re}(s)>-1 / 2+\max \{-2 \operatorname{Re}(\omega), 0\}$. Let $1 \leq y \leq M$ and $R$ be a polynomial with $R(0)=R^{\prime}(0)=0$. Set

$$
E(r):=\prod_{p \mid r}(1+1 / \sqrt{p}) .
$$

For any positive integer $r \leq y$ we have

$$
\begin{aligned}
T_{(\alpha, \beta)}(y, R ; r ; z)= & \operatorname{Res}_{s=0} \frac{\mu(r) G_{(\alpha, \beta)}(s ; r ; z) \zeta(1+s+2 z)}{s \zeta(1+s+z+\alpha) \zeta(1+s+z+\beta)} \sum_{j=0}^{\infty} \frac{1}{(s \log y)^{j}} R^{(j)}\left(\frac{\log y / r}{\log y}\right) \\
& +\delta_{z \notin\{\alpha, \beta\}} \frac{\mu(r) G_{(\alpha, \beta)}(-2 z ; r ; z)}{\zeta(1-z+\alpha) \zeta(1-z+\beta)}\left(\frac{y}{r}\right)^{-2 z} \sum_{j=2}^{\infty} \frac{R^{(j)}(0)}{(-2 z)^{j+1} \log ^{j} y} \\
& +\mathcal{O}\left(\frac{E(r)}{\log ^{2} y} e^{-A_{0} \sqrt{\log y / r}}\right),
\end{aligned}
$$

where $\delta_{z \notin\{\alpha, \beta\}}$ is 1 if $z \notin\{\alpha, \beta\}$, and 0 , otherwise.

Proof. The first equation follows from comparing two Euler products. Indeed, we have

$$
\begin{aligned}
T_{(\alpha, \beta)}(s ; r ; z) & =\sum_{m \geq 1}^{b} \frac{1}{m^{1+s+z}} \sum_{\ell n=m} \frac{\nu_{(\alpha, \beta)}(\ell) \mu^{2}(\ell n r) \mu(\ell r)}{n^{z}} \\
& =\mu(r) \sum_{\substack{m \geq 1 \\
(m, r)=1}}^{b} \frac{1}{m^{1+s+z}} \sum_{\ell n=m} \frac{\eta_{(\alpha-\beta) / 2}(\ell) \mu(\ell)}{\ell^{(\alpha+\beta) / 2} n^{z}} \\
& =\mu(r) \prod_{p \nmid r}\left(1-\frac{1}{p^{1+s+z+\alpha}}-\frac{1}{p^{1+s+z+\beta}}+\frac{1}{p^{1+s+2 z}}\right),
\end{aligned}
$$

where $b$ means that we sum over square-free integers. To prove the other statement, we will use the identity

$$
\frac{1}{2 \pi i} \int_{3-i \infty}^{3+i \infty} y^{s} \frac{d s}{s^{k+2}}=\delta_{y \geq 1} \frac{\log ^{k+1}(y)}{(k+1) !}
$$

which is standard by using suitable contour shifts. By the Taylor expansion $R(x)=$ $\sum_{j=2}^{\infty} \frac{R^{(j)}(0)}{j !} x^{j}$ (here we use the assumption $R(0)=R^{\prime}(0)=0$ ), we see that

$$
\begin{aligned}
T_{(\alpha, \beta)}(y, R ; r ; z) & =\sum_{\ell \geq 1} \frac{\nu_{(\alpha, \beta)}(\ell)}{\ell^{1+z}} \sum_{1 \leq n \leq y /(\ell r)} \frac{\mu^{2}(\ell n r) \mu(\ell r)}{n^{1+2 z}} \sum_{j=2}^{\infty} \frac{R^{(j)}(0)}{j !}\left(\frac{\log (y /(\ell n r))}{\log y}\right)^{j} \\
& =\sum_{j=2}^{\infty} \frac{R^{(j)}(0)}{j ! \log ^{j} y} \sum_{\ell \geq 1} \frac{\nu_{(\alpha, \beta)}(\ell)}{\ell^{1+z}} \sum_{1 \leq n \leq y /(\ell r)} \frac{\mu^{2}(\ell n r) \mu(\ell r)}{n^{1+2 z}} \log ^{j}(y /(\ell n r)) \\
& =\sum_{j=2}^{\infty} \frac{R^{(j)}(0)}{\log ^{j} y} \frac{1}{2 \pi i} \int_{3-i \infty}^{3+i \infty} \sum_{\ell \geq 1} \frac{\nu_{(\alpha, \beta)}(\ell)}{\ell^{1+s+z}} \sum_{n \geq 1} \frac{\mu^{2}(\ell n r) \mu(\ell r)}{n^{1+s+2 z}}\left(\frac{y}{r}\right)^{s} \frac{d s}{s^{j+1}}
\end{aligned}
$$




$$
=\sum_{j=2}^{\infty} \frac{R^{(j)}(0)}{\log ^{j} y} \frac{1}{2 \pi i} \int_{3-i \infty}^{3+i \infty} \frac{\mu(r) G_{(\alpha, \beta)}(s ; r ; z) \zeta(1+s+2 z)}{\zeta(1+s+z+\alpha) \zeta(1+s+z+\beta)}\left(\frac{y}{r}\right)^{s} \frac{d s}{s^{j+1}} .
$$

We may evaluate the above integral by a standard procedure. First shift the contour to $\operatorname{Re}(s)=\frac{B}{\log (2 y / r)}$, and truncate the integral to the line segment from $\frac{B}{\log (2 y / r)}-i T$ to $\frac{B}{\log (2 y / r)}+i T$ where $B$ is a constant such that $\frac{B}{\log (2 y / r)}>-2 \delta$, and

$$
T:=\exp (\sqrt{\log (2 y / r)}) .
$$

The error involved in doing so is

$$
\ll \frac{E(r)}{\log ^{2} y} \log ^{3}(2 y / r) T^{-2} .
$$

Next, shift the integral on this line segment to the left onto the line segment $-c / \log T$, where $c$ is a positive constant such that $\zeta(1+s)$ has no zeros in the region $\operatorname{Re}(s)>$ $-c / \log T$ and $\operatorname{Im}(s) \leq T$. We encounter a multiple pole at $s=0$, and another simple pole at $s=-2 z$ if $z \notin\{\alpha, \beta\}$. The integrals on the three other sides are bounded using standard estimates for $1 / \zeta(s)$ in the zero-free region, and contribute an amount

$$
\ll \frac{E(r)}{\log ^{2} y}\left(\log \frac{T y}{r}\right)^{3}\left(T^{-2}+(y / r)^{-c / \log T}\right) .
$$

We conclude that for an appropriate positive constant $A_{0}$, we have

$$
\begin{aligned}
T_{(\alpha, \beta)}(y, R ; r ; z)= & \operatorname{Res}_{s=0} \frac{\mu(r) G_{(\alpha, \beta)}(s ; r ; z) \zeta(1+s+2 z)}{s \zeta(1+s+z+\alpha) \zeta(1+s+z+\beta)} \sum_{j=2}^{\infty} \frac{R^{(j)}(0)}{s^{j} \log ^{j} y}\left(\frac{y}{r}\right)^{s} \\
& +\operatorname{Res}_{s=-2 z} \frac{\mu(r) G_{(\alpha, \beta)}(s ; r ; z) \zeta(1+s+2 z)}{\zeta(1+s+z+\alpha) \zeta(1+s+z+\beta)}\left(\frac{y}{r}\right)^{s} \sum_{j=2}^{\infty} \frac{R^{(j)}(0)}{s^{j+1} \log ^{j} y} \\
& +\mathcal{O}\left(\frac{E(r)}{\log ^{2} y} e^{-A_{0} \sqrt{\log y / r}}\right) .
\end{aligned}
$$

Now we will follow the same argument as in Conrey-Soundararajan [2, p. 38] for the residue at $s=0$. Indeed, we may replace $\sum_{j=2}^{\infty} \frac{R^{(j)}(0)}{s^{j} \log ^{j} y}\left(\frac{y}{r}\right)^{s}$ with

$$
\begin{aligned}
\sum_{j=2}^{\infty} \frac{R^{(j)}(0)}{s^{j} \log ^{j} y}\left(\sum_{l \leq j} \frac{(s \log y / r)^{l}}{l !}\right) & =\sum_{k=0}^{\infty} \frac{1}{(s \log y)^{k}} \sum_{l=0}^{\infty} \frac{R^{(k+l)}(0)}{l !}\left(\frac{\log (y / r)}{\log y}\right)^{l} \\
& =\sum_{j=0}^{\infty} \frac{1}{(s \log y)^{j}} R^{(j)}\left(\frac{\log (y / r)}{\log y}\right)
\end{aligned}
$$

by grouping terms according to $k=j-l$, and using the fact $R(0)=R^{\prime}(0)=0$. This completes the proof of the lemma.

5.20. Contribution of the short-range terms. Recall that $0<\Upsilon<1$ is fixed. We first use Lemma 5.19 and equation (5.17) to deduce the estimate for $S_{(\alpha, \beta)}(r ; z)$ when $r \leq M^{1-\Upsilon}$. 
5.21. The case $(\alpha, \beta)=(\omega, \bar{\omega})$. In this case we have

$$
S_{(\omega, \bar{\omega})}(r ; z)=\frac{\mu(r) G_{(\omega, \bar{\omega})}(0 ; r ; z) \zeta(1+2 z)}{\zeta(1+z+\omega) \zeta(1+z+\bar{\omega})}+\mathcal{O}\left(\frac{E(r)}{\log ^{2} K} e^{-A_{0} \sqrt{\left(\log M^{1-\Upsilon}\right) / r}}\right) .
$$

Note that the main term above came form the $j=0$ contribution in the applications of Lemma 5.19, and that the contributions from $j \geq 1$ in the two applications cancel each other. By (5.12), we have

$$
\begin{aligned}
\mathcal{V}_{(\omega, \bar{\omega})}^{\leq}(\omega)= & \sum_{1 \leq r \leq M^{1-\Upsilon}} \frac{\mu(r)^{2}}{r^{1+\omega+\bar{\omega}}} \tau_{(\omega, \bar{\omega})}(r) \prod_{z \in\{\omega, \bar{\omega}\}} \frac{G_{(\omega, \bar{\omega})}(0 ; r ; z) \zeta(1+2 z)}{\zeta(1+z+\omega) \zeta(1+z+\bar{\omega})} \\
& +\mathcal{O}\left(\frac{|\omega+\bar{\omega}|}{(\log K)^{2}}\right) .
\end{aligned}
$$

Indeed, here we use the fact

$$
\sum_{1 \leq r \leq x}^{b} E(r)^{3} \ll x, \quad \text { and, therefore, } \quad \sum_{1 \leq r \leq x}^{b} \frac{E(r)^{3}}{r} e^{-c \sqrt{\log x / r}} \ll 1 .
$$

Hence, by 5.5 and (5.7), we have

$$
\begin{aligned}
\Psi(\omega, \bar{\omega}) \mathcal{V}_{(\omega, \bar{\omega})}^{\leq}(\omega)= & \frac{K}{4} \frac{\widetilde{\Phi}(1)}{\zeta(1+\omega+\bar{\omega})} \sum_{1 \leq r \leq M^{1-\Upsilon}} \frac{\mu(r)^{2} \tau_{(\omega, \bar{\omega})}(r) G_{(\omega, \bar{\omega})}(0 ; r ; \omega) G_{(\omega, \bar{\omega})}(0 ; r ; \bar{\omega})}{r^{1+\omega+\bar{\omega}}} \\
& +\mathcal{O}\left(\frac{K}{(\log K)^{2}}\right) .
\end{aligned}
$$

To deal with the innermost sum above, we will use Perron's formula to prove the following lemma.

5.22. Lemma. Assume that $\log x \asymp \log K$ and $\omega$ satisfies $(\mathbf{I})$. Then

$$
\begin{aligned}
& \sum_{1 \leq r \leq x} \frac{\mu(r)^{2} \tau_{(\omega, \bar{\omega})}(r) G_{(\omega, \bar{\omega})}(0 ; r ; \omega) G_{(\omega, \bar{\omega})}(0 ; r ; \bar{\omega})}{r^{1+\omega+\bar{\omega}}} \\
& =\zeta(1+\omega+\bar{\omega})\left(1-x^{-(\omega+\bar{\omega})}\right)(1+\mathcal{O}(|\omega+\bar{\omega}|))+\mathcal{O}\left(x^{-(\omega+\bar{\omega})}\right) .
\end{aligned}
$$

Furthermore, if $1 \leq y \leq x$ and $\log y \asymp \log x$ then for any smooth function $R$ on $[0,1]$, we have

$$
\begin{array}{r}
\sum_{y \leq r \leq x} \frac{\mu(r)^{2} \tau_{(\omega, \bar{\omega})}(r) G_{(\omega, \bar{\omega})}(0 ; r ; \omega) G_{(\omega, \bar{\omega})}(0 ; r ; \bar{\omega})}{r^{1+\omega+\bar{\omega}}} R\left(\frac{\log r}{\log x}\right) \\
=(1+\mathcal{O}(|\omega+\bar{\omega}|)) \int_{y}^{x} R\left(\frac{\log t}{\log x}\right) \frac{d t}{t^{1+\omega+\bar{\omega}}}+\mathcal{O}_{R}(1) .
\end{array}
$$

Proof. Recalling the definition of $G_{(\omega, \bar{\omega})}(0 ; r ; \omega)$ in Lemma 5.19 for any square-free $r$, we have

$$
G_{(\omega, \bar{\omega})}(0 ; r ; z)=G_{(\omega, \bar{\omega})}(0 ; 1 ; z) \prod_{p \mid r}\left(1-\frac{1}{p^{1+\omega+\bar{\omega}}}\right)^{-1}=\prod_{p \mid r}\left(1-\frac{1}{p^{1+\omega+\bar{\omega}}}\right)^{-1}
$$


where we use the fact that $G_{(\omega, \bar{\omega})}(0 ; 1 ; z)=1$ for $z \in\{\omega, \bar{\omega}\}$. Hence

$$
\begin{aligned}
\sum_{r} \frac{\mu(r)^{2} \tau_{(\omega, \bar{\omega})}(r) G_{(\omega, \bar{\omega})}(0 ; r ; \omega) G_{(\omega, \bar{\omega})}(0 ; r ; \bar{\omega})}{r^{1+s+\omega+\bar{\omega}}} & \\
= & \sum_{r} \frac{\mu(r)^{2} \tau_{(\omega, \bar{\omega})}(r) \prod_{p \mid r}\left(1-1 / p^{1+\omega+\bar{\omega}}\right)^{-2}}{r^{1+s+\omega+\bar{\omega}}} \\
& =\prod_{p}\left(1+\frac{\left(1-1 / p^{1+\omega+\bar{\omega}}\right)\left(1-1 / p^{1+\omega+\bar{\omega}}\right)^{-2}}{p^{1+s+\omega+\bar{\omega}}}\right) \\
& =\zeta(1+s+\omega+\bar{\omega}) \prod_{p}\left(1-\frac{1}{p^{1+s+\omega+\bar{\omega}}}\right)\left(1+\sum_{k=1}^{\infty} \frac{1}{p^{s+k(1+\omega+\bar{\omega})}}\right) \\
& =: \zeta(1+s+\omega+\bar{\omega}) H_{(\omega, \bar{\omega})}(s) .
\end{aligned}
$$

We know that $H_{(\omega, \bar{\omega})}(s)$ is holomorphic for $\operatorname{Re}(s)>-1+2|\delta|$, and $H_{(\omega, \bar{\omega})}(0)=1$. Now we can use Perron's formula to conclude our first statement. And the second statement will follow by the partial summation formula. Indeed, by Perron's formula (see [22, Lemma 3.12]), we have

$$
\begin{aligned}
& \sum_{1 \leq r \leq x} \frac{\mu(r)^{2} \tau_{(\omega, \bar{\omega})}(r) G_{(\omega, \bar{\omega})}(0 ; r ; \omega) G_{(\omega, \bar{\omega})}(0 ; r ; \bar{\omega})}{r^{1+\omega+\bar{\omega}}} \\
& \quad=\frac{1}{2 \pi i} \int_{-2 \delta+A / \log x-i T}^{-2 \delta+A / \log x+i T} \zeta(1+s+\omega+\bar{\omega}) H_{(\omega, \bar{\omega})}(s) x^{s} \frac{d s}{s}+\mathcal{O}\left(\frac{x^{-2 \delta}(\log x)^{B}}{T}\right),
\end{aligned}
$$

where constants $A, B>0$ satisfy $-2 \delta+\frac{A}{\log x}>0$ and

$$
\tau_{(\omega, \bar{\omega})}(r) G_{(\omega, \bar{\omega})}(0 ; r ; \omega) G_{(\omega, \bar{\omega})}(0 ; r ; \bar{\omega})=\mathcal{O}\left((\log r)^{B}\right)
$$

We further choose $T=e^{\sqrt{\log x}}$. Now shift the contour to the line segment $-2 \delta-\frac{C}{\log T}-i T$ to $-2 \delta-\frac{C}{\log T}+i T$, getting

$$
\begin{aligned}
& \sum_{1 \leq r \leq x} \frac{\mu(r)^{2} \tau_{(\alpha, \beta)}(r) G_{(\omega, \bar{\omega})}(0 ; r ; \omega) G_{(\omega, \bar{\omega})}(0 ; r ; \bar{\omega})}{r^{1+\omega+\bar{\omega}}} \\
& =\operatorname{Res}_{s=0}+\operatorname{Res}_{s=-(\omega+\bar{\omega})}+\mathcal{O}\left(\left|\frac{1}{2 \pi i} \int_{-2 \delta-C / \log T-i T}^{-2 \delta-C / \log T+i T} \zeta(1+s+\omega+\bar{\omega}) H_{(\omega, \bar{\omega})}(s) x^{s} \frac{d s}{s}\right|\right) \\
& \quad+\mathcal{O}\left(\left|\frac{1}{2 \pi i} \int_{-2 \delta-C / \log T \pm i T}^{-2 \delta+A / \log x \pm i T} \zeta(1+s+\omega+\bar{\omega}) H_{(\omega, \bar{\omega})}(s) x^{s} \frac{d s}{s}\right|\right)+\mathcal{O}\left(x^{-2 \delta}\right) \\
& =\zeta(1+\omega+\bar{\omega})\left(1-x^{-(\omega+\bar{\omega})}\right)(1+\mathcal{O}(|\omega+\bar{\omega}|))+\mathcal{O}\left(x^{-(\omega+\bar{\omega})}\right) \\
& =\zeta(1+\omega+\bar{\omega})\left(1-x^{-(\omega+\bar{\omega})}\right)+\mathcal{O}(1) .
\end{aligned}
$$


This proves the first claim of the lemma. For our second assertion, we use partial summation, obtaining

$$
\begin{aligned}
& \sum_{y \leq r \leq x} \frac{\mu(r)^{2} \tau_{(\omega, \bar{\omega})}(r) G_{(\omega, \bar{\omega})}(0 ; r ; \omega) G_{(\omega, \bar{\omega})}(0 ; r ; \bar{\omega})}{r^{1+\omega+\bar{\omega}}} R\left(\frac{\log r}{\log x}\right) \\
&=\int_{y}^{x} R\left(\frac{\log t}{\log x}\right) d \sum_{1 \leq r \leq t} \frac{\mu(r)^{2} \tau_{(\omega, \bar{\omega})}(r) G_{(\omega, \bar{\omega})}(0 ; r ; \omega) G_{(\omega, \bar{\omega})}(0 ; r ; \bar{\omega})}{r^{1+\omega+\bar{\omega}}} \\
&=\left.R\left(\frac{\log t}{\log x}\right)\left(\sum_{1 \leq r \leq t} \frac{\mu(r)^{2} \tau_{(\omega, \bar{\omega})}(r) G_{(\omega, \bar{\omega})}(0 ; r ; \omega) G_{(\omega, \bar{\omega})}(0 ; r ; \bar{\omega})}{r^{1+\omega+\bar{\omega}}}\right)\right|_{y} ^{x} \\
&-\int_{y}^{x}\left(\sum_{1 \leq r \leq t} \frac{\mu(r)^{2} \tau_{(\omega, \bar{\omega})}(r) G_{(\omega, \bar{\omega})}(0 ; r ; \omega) G_{(\omega, \bar{\omega})}(0 ; r ; \bar{\omega})}{r^{1+\omega+\bar{\omega}}}\right) d R\left(\frac{\log t}{\log x}\right) \\
&=\left.R\left(\frac{\log t}{\log x}\right) \zeta(1+\omega+\bar{\omega})\left(1-t^{-(\omega+\bar{\omega})}\right)\right|_{y} ^{x} \\
&-\int_{y}^{x} \zeta(1+\omega+\bar{\omega})\left(1-t^{-(\omega+\bar{\omega})}\right) d R\left(\frac{\log t}{\log x}\right)+\mathcal{O}_{R}(1) \\
&=(1+\mathcal{O}(|\omega+\bar{\omega}|)) \int_{y}^{x} R\left(\frac{\log t}{\log x}\right) \frac{d t}{t^{1+\omega+\bar{\omega}}+\mathcal{O}_{R}(1) .}
\end{aligned}
$$

This completes the proof of the lemma.

As a consequence of Lemma 5.22, we obtain

$$
\begin{aligned}
& \Psi(\omega, \bar{\omega}) \mathcal{V}_{(\omega, \bar{\omega})}^{\leq}(\omega) \\
& \quad=\frac{K}{4} \widetilde{\Phi}(1)\left(1-M^{-(1+\Upsilon)(\omega+\bar{\omega})}\right)(1+\mathcal{O}(|\omega+\bar{\omega}|))+\mathcal{O}\left(\frac{K}{(\log K)^{2}}\right) .
\end{aligned}
$$

5.24. The case $(\alpha, \beta)=(-\omega,-\bar{\omega})$. Here we have

$$
\begin{aligned}
S_{(-\omega,-\bar{\omega})}(r ; z)= & \frac{\mu(r) G_{(-\omega,-\bar{\omega})}(-2 z ; r ; z) r^{2 z}}{\zeta(1-z-\omega) \zeta(1-z-\bar{\omega})} \\
& \times\left(M^{-2 z} \sum_{j=2}^{\infty} \frac{P^{(j)}(0)}{(-2 z)^{j+1} \log ^{j} M}+M^{-2 z(1-\Upsilon)} \sum_{j=2}^{\infty} \frac{Q^{(j)}(0)}{(-2 z)^{j+1} \log ^{j} M^{1-\Upsilon}}\right) \\
& +\mathcal{O}\left(\frac{E(r)}{\log ^{2} K} e^{-A_{0} \sqrt{\left(\log M^{1-\Upsilon}\right) / r}}\right) .
\end{aligned}
$$

It follows from 5.12 that

$$
\begin{aligned}
\mathcal{V}_{(-\omega,-\bar{\omega})}^{\leq}(\omega)= & \sum_{1 \leq r \leq M^{1-\Upsilon}} \frac{\mu(r)^{2} \tau_{(-\omega,-\bar{\omega})}(r) G_{(-\omega,-\bar{\omega})}(-2 \omega ; r ; \omega) G_{(-\omega,-\bar{\omega})}(-2 \bar{\omega} ; r ; \bar{\omega})}{r^{1-\omega-\bar{\omega}} \zeta(1-2 \omega) \zeta(1-2 \bar{\omega}) \zeta(1-\omega-\bar{\omega})^{2}} \\
& \times\left|M^{-2 \omega} \sum_{j=2}^{\infty} \frac{P^{(j)}(0)}{(-2 \omega)^{j+1} \log ^{j} M}+M^{-2 \omega(1-\Upsilon)} \sum_{j=2}^{\infty} \frac{Q^{(j)}(0)}{(-2 \omega)^{j+1} \log ^{j} M^{1-\Upsilon}}\right|^{2} \\
& +\mathcal{O}\left(\frac{|\omega+\bar{\omega}|(\log \log K)^{3}}{(\log K)^{2}}\right) .
\end{aligned}
$$


Hence

$$
\begin{aligned}
\Psi(-\omega,-\bar{\omega}) \mathcal{V}_{(-\omega,-\bar{\omega})}^{\leq} & (\omega)=\frac{K}{4}\left(\frac{K}{4 \pi}\right)^{-2 \omega-2 \bar{\omega}} \frac{\widetilde{\Phi}(1)(1+\mathcal{O}(|\omega|))}{\zeta(1-\omega-\bar{\omega})} \\
& \times \sum_{1 \leq r \leq M^{1-\Upsilon}} \frac{\mu(r)^{2} \tau_{(-\omega,-\bar{\omega})}(r) G_{(-\omega,-\bar{\omega})}(-2 \omega ; r ; \omega) G_{(-\omega,-\bar{\omega})}(-2 \bar{\omega} ; r ; \bar{\omega})}{r^{1-\omega-\bar{\omega}}} \\
& \times\left|M^{-2 \omega} \sum_{j=2}^{\infty} \frac{P^{(j)}(0)}{(-2 \omega)^{j} \log ^{j} M}+M^{-2 \omega(1-\Upsilon)} \sum_{j=2}^{\infty} \frac{Q^{(j)}(0)}{(-2 \omega)^{j} \log ^{j} M^{1-\Upsilon}}\right|^{2} \\
& +\mathcal{O}\left(\frac{(\log \log K)^{3}}{(\log K)^{2}} K\right) .
\end{aligned}
$$

To deal with the $r$-sum above, we will use Perron's formula again to prove the following lemma.

5.25. Lemma. Assume that $\log x \asymp \log K$ and $\omega$ satisfies $(\mathbf{I})$. We have

$$
\begin{aligned}
& \sum_{1 \leq r \leq x} \frac{\mu(r)^{2} \tau_{(-\omega,-\bar{\omega})}(r) G_{(-\omega,-\bar{\omega})}(-2 \omega ; r ; \omega) G_{(-\omega,-\bar{\omega})}(-2 \bar{\omega} ; r ; \bar{\omega})}{r^{1-\omega-\bar{\omega}}} \\
&=\zeta(1-\omega-\bar{\omega})\left(1-x^{\omega+\bar{\omega}}\right)(1+\mathcal{O}(|\omega|))+\mathcal{O}\left(e^{-c \sqrt{\log x}}\right) .
\end{aligned}
$$

Furthermore, if $1 \leq y \leq x$ and $\log y \asymp \log x$ then for any smooth function $R$ on $[0,1]$, we have

$$
\begin{array}{r}
\sum_{y \leq r \leq x} \frac{\mu(r)^{2} \tau_{(-\omega,-\bar{\omega})}(r) G_{(-\omega,-\bar{\omega})}(-2 \omega ; r ; \omega) G_{(-\omega,-\bar{\omega})}(-2 \bar{\omega} ; r ; \bar{\omega})}{r^{1-\omega-\bar{\omega}}} R\left(\frac{\log r}{\log x}\right) \\
=(1+\mathcal{O}(|\omega+\bar{\omega}|)) \int_{y}^{x} R\left(\frac{\log t}{\log x}\right) \frac{d t}{t^{1-\omega-\bar{\omega}}}+\mathcal{O}_{R}\left(x^{\omega+\bar{\omega}}\right) .
\end{array}
$$

Similarly

$$
\begin{array}{r}
\sum_{y<r \leq x} \frac{\mu(r)^{2} \tau_{(-\omega,-\bar{\omega})}(r) G_{(-\omega,-\bar{\omega})}(0 ; r ; \omega) G_{(-\omega,-\bar{\omega})}(0 ; r ; \bar{\omega})}{r^{1+\omega+\bar{\omega}}} R\left(\frac{\log r}{\log x}\right) \\
=(1+\mathcal{O}(|\omega+\bar{\omega}|)) \int_{y}^{x} R\left(\frac{\log t}{\log x}\right) \frac{d t}{t^{1+\omega+\bar{\omega}}}+\mathcal{O}_{R}\left((\log \log K)^{2}\right),
\end{array}
$$

and

$$
\begin{array}{r}
\sum_{y<r \leq x} \frac{\mu(r)^{2} \tau_{(-\omega,-\bar{\omega})}(r) G_{(-\omega,-\bar{\omega})}(0 ; r ; \omega) G_{(-\omega,-\bar{\omega})}(-2 \bar{\omega} ; r ; \bar{\omega})}{r^{1+\omega-\bar{\omega}}} R\left(\frac{\log r}{\log x}\right) \\
=(1+\mathcal{O}(|\omega-\bar{\omega}|)) \int_{y}^{x} R\left(\frac{\log t}{\log x}\right) \frac{d t}{t^{1+\omega-\bar{\omega}}}+\mathcal{O}_{R}\left((\log \log K)^{2}\right) .
\end{array}
$$

Proof. Recalling the definition of $G_{(-\omega,-\bar{\omega})}(-2 \omega ; r ; \omega)$ in Lemma 5.19 we have

$$
G_{(-\omega,-\bar{\omega})}(-2 z ; r ; z)=G_{(-\omega,-\bar{\omega})}(-2 z ; 1 ; z) \prod_{p \mid r}\left(1-\frac{1}{p^{1-z-\omega}}-\frac{1}{p^{1-z-\bar{\omega}}}+\frac{1}{p}\right)^{-1}
$$


where $G_{(-\omega,-\bar{\omega})}(-2 z ; 1 ; z)=1+\mathcal{O}(|\omega|)$ for $z \in\{\omega, \bar{\omega}\}$. Hence

$$
\begin{aligned}
\sum_{r} \frac{\mu(r)^{2}}{\tau_{(-\omega,-\bar{\omega})}(r) G_{(-\omega,-\bar{\omega})}(-2 \omega ; r ; \omega) G_{(-\omega,-\bar{\omega})}(-2 \bar{\omega} ; r ; \bar{\omega})} & r^{1+s-\omega-\bar{\omega}} \\
= & \prod_{z \in\{\omega, \bar{\omega}\}} G_{(-\omega,-\bar{\omega})}(-2 z ; 1 ; z) \\
& \times \sum_{r} \frac{\mu(r)^{2} \tau_{(-\omega,-\bar{\omega})}(r) \prod_{z \in\{\omega, \bar{\omega}\}} \prod_{p \mid r}\left(1-1 / p^{1-z-\omega}-1 / p^{1-z-\bar{\omega}}+1 / p\right)^{-1}}{r^{1+s-\omega-\bar{\omega}}} \\
= & \prod_{z \in\{\omega, \bar{\omega}\}} G_{(-\omega,-\bar{\omega})}(-2 z ; 1 ; z) \\
& \times \prod_{p}\left(1+\frac{\tau_{(-\omega,-\bar{\omega})}(p) \prod_{z \in\{\omega, \bar{\omega}\}}\left(1-1 / p^{1-z-\omega}-1 / p^{1-z-\bar{\omega}}+1 / p\right)^{-1}}{p^{1+s-\omega-\bar{\omega}}}\right) \\
= & : \zeta(1+s-\omega-\bar{\omega}) H_{(-\omega,-\bar{\omega})}(s),
\end{aligned}
$$

where $H_{(-\omega,-\bar{\omega})}(s)$ is holomorphic for $\operatorname{Re}(s)>-1+2|\delta|$, and $H_{(-\omega,-\bar{\omega})}(0)=1+\mathcal{O}(|\omega|)$. Now we can use Perron's formula to obtain the first statement in Lemma 5.25. The second statement follows by the partial summation formula.

It follows from Lemma 5.25 that

$$
\begin{aligned}
\Psi(-\omega,-\bar{\omega}) & \mathcal{V}_{(-\omega,-\bar{\omega})}^{\leq}(\omega) \\
= & \frac{K}{4}\left(\frac{K}{4 \pi}\right)^{-2 \omega-2 \bar{\omega}} \widetilde{\Phi}(1)\left(1-M^{(\omega+\bar{\omega})(1-\Upsilon)}\right)(1+\mathcal{O}(|\omega|)) \\
& \times\left|M^{-2 \omega} \sum_{j=2}^{\infty} \frac{P^{(j)}(0)}{(-2 \omega)^{j} \log ^{j} M}+M^{-2 \omega(1-\Upsilon)} \sum_{j=2}^{\infty} \frac{Q^{(j)}(0)}{(-2 \omega)^{j} \log ^{j} M^{1-\Upsilon}}\right|^{2} \\
& +\mathcal{O}\left(\frac{(\log \log K)^{3}}{(\log K)^{2}} K\right) .
\end{aligned}
$$

5.27. The cases $(\alpha, \beta)=(\omega,-\bar{\omega})$ and $(\alpha, \beta)=(-\omega, \bar{\omega})$. We first consider $(\alpha, \beta)=$ $(\omega,-\bar{\omega})$. By Lemma 5.19 , we have

$$
S_{(\omega,-\bar{\omega})}(r ; \omega)=\frac{\mu(r) G_{(\omega,-\bar{\omega})}(0 ; r ; \omega)}{\zeta(1+\omega-\bar{\omega})}+\mathcal{O}\left(\frac{E(r)}{\log ^{2} K} e^{-A_{0} \sqrt{\left(\log M^{1-\Upsilon}\right) / r}}\right),
$$

and

$$
\begin{aligned}
S_{(\omega,-\bar{\omega})}( & ; \bar{\omega})=\frac{\mu(r) G_{(\omega,-\bar{\omega})}(-2 \bar{\omega} ; r ; \bar{\omega}) r^{2 \bar{\omega}}}{\zeta(1-\bar{\omega}+\omega) \zeta(1-2 \bar{\omega})} \\
& \times\left(M^{-2 \bar{\omega}} \sum_{j=2}^{\infty} \frac{P^{(j)}(0)}{(-2 \bar{\omega})^{j+1}(\log M)^{j}}+M^{-2 \bar{\omega}(1-\Upsilon)} \sum_{j=2}^{\infty} \frac{Q^{(j)}(0)}{(-2 \bar{\omega})^{j+1}\left(\log M^{1-\Upsilon}\right)^{j}}\right) \\
& +\mathcal{O}\left(\frac{E(r)}{\log ^{2} K} e^{-A_{0} \sqrt{\left(\log M^{1-\Upsilon}\right) / r}}\right) .
\end{aligned}
$$


By 5.12 , we have

$$
\begin{aligned}
\mathcal{V}_{(\omega,-\bar{\omega})}^{\leq}(\omega) & =\sum_{1 \leq r \leq M^{1-\Upsilon}} \frac{\mu(r)^{2} \tau_{(\omega,-\bar{\omega})}(r) G_{(\omega,-\bar{\omega})}(0 ; r ; \omega) G_{(\omega,-\bar{\omega})}(-2 \bar{\omega} ; r ; \bar{\omega})}{r^{1+\omega-\bar{\omega}} \zeta(1-2 \bar{\omega}) \zeta(1+\omega-\bar{\omega})^{2}} \\
& \times\left(M^{-2 \bar{\omega}} \sum_{j=2}^{\infty} \frac{P^{(j)}(0)}{(-2 \bar{\omega})^{j+1}(\log M)^{j}}+M^{-2 \bar{\omega}(1-\Upsilon)} \sum_{j=2}^{\infty} \frac{Q^{(j)}(0)}{(-2 \bar{\omega})^{j+1}\left(\log M^{1-\Upsilon}\right)^{j}}\right) \\
& +\mathcal{O}\left(\frac{|\omega-\bar{\omega}|(\log \log K)^{3}}{(\log K)^{2}}\right) .
\end{aligned}
$$

Hence

$$
\begin{aligned}
\Psi(\omega,-\bar{\omega}) \mathcal{V}_{(\omega,-\bar{\omega})}^{\leq} & (\omega) \\
= & -\frac{K}{4}\left(\frac{K}{4 \pi}\right)^{-2 \bar{\omega}} \frac{\widetilde{\Phi}(1)}{\zeta(1+\omega-\bar{\omega})}(1+\mathcal{O}(|\omega|)) \\
& \times \sum_{1 \leq r \leq M^{1-\Upsilon}} \frac{\mu(r)^{2} \tau_{(\omega,-\bar{\omega})}(r) G_{(\omega,-\bar{\omega})}(0 ; r ; \omega) G_{(\omega,-\bar{\omega})}(-2 \bar{\omega} ; r ; \bar{\omega})}{r^{1+\omega-\bar{\omega}}} \\
& \times\left(M^{-2 \bar{\omega}} \sum_{j=2}^{\infty} \frac{P^{(j)}(0)}{(-2 \bar{\omega})^{j}(\log M)^{j}}+M^{-2 \bar{\omega}(1-\Upsilon)} \sum_{j=2}^{\infty} \frac{Q^{(j)}(0)}{(-2 \bar{\omega})^{j}\left(\log M^{1-\Upsilon}\right)^{j}}\right) \\
& +\mathcal{O}\left(\frac{(\log \log K)^{3}}{(\log K)^{2}} K\right) .
\end{aligned}
$$

To deal with the $r$-sum above, we will use Perron's formula again to prove the following lemma.

5.28. Lemma. Assume that $\log x \asymp \log K$ and $\omega$ satisfies $(\mathbf{I})$. We have

$$
\begin{aligned}
& \sum_{1 \leq r \leq x} \frac{\mu(r)^{2} \tau_{(\omega,-\bar{\omega})}(r) G_{(\omega,-\bar{\omega})}(0 ; r ; \omega) G_{(\omega,-\bar{\omega})}(-2 \bar{\omega} ; r ; \bar{\omega})}{r^{1+\omega-\bar{\omega}}} \\
& \quad=\zeta(1+\omega-\bar{\omega})\left(1-x^{-(\omega-\bar{\omega})}\right)(1+\mathcal{O}(|\omega|))+\mathcal{O}(1) .
\end{aligned}
$$

Furthermore, if $1 \leq y \leq x$ and $\log y \asymp \log x$ then for any smooth function $R$ on $[0,1]$

$$
\begin{aligned}
\sum_{y \leq r \leq x} \frac{\mu(r)^{2} \tau_{(\omega,-\bar{\omega})}(r) G_{(\omega,-\bar{\omega})}(0 ; r ; \omega) G_{(\omega,-\bar{\omega})}(-2 \bar{\omega} ; r ; \bar{\omega})}{r^{1+\omega-\bar{\omega}}} R\left(\frac{\log r}{\log x}\right) \\
=(1+\mathcal{O}(|\omega-\bar{\omega}|)) \int_{y}^{x} R\left(\frac{\log t}{\log x}\right) \frac{d t}{t^{1+\omega-\bar{\omega}}}+\mathcal{O}_{R}\left((\log \log K)^{2}\right) .
\end{aligned}
$$

Similarly, we have

$$
\begin{aligned}
\sum_{y<r \leq x} \frac{\mu(r)^{2} \tau_{(\omega,-\bar{\omega})}(r) G_{(\omega,-\bar{\omega})}(0 ; r ; \omega) G_{(\omega,-\bar{\omega})}(0 ; r ; \bar{\omega})}{r^{1+\omega+\bar{\omega}}} R\left(\frac{\log r}{\log x}\right) \\
=(1+\mathcal{O}(|\omega+\bar{\omega}|)) \int_{y}^{x} R\left(\frac{\log t}{\log x}\right) \frac{d t}{t^{1+\omega+\bar{\omega}}}+\mathcal{O}_{R}\left((\log \log K)^{2}\right) .
\end{aligned}
$$


Proof. Recalling the definition of $G_{(\omega,-\bar{\omega})}(0 ; r ; \omega)$ and $G_{(\omega,-\bar{\omega})}(-2 \bar{\omega} ; r ; \bar{\omega})$ in Lemma 5.19 , we have

$$
\begin{aligned}
G_{(\omega,-\bar{\omega})}(0 ; r ; \omega) & =G_{(\omega,-\bar{\omega})}(0 ; 1 ; \omega) \prod_{p \mid r}\left(1-\frac{1}{p^{1+\omega-\bar{\omega}}}\right)^{-1} \\
G_{(\omega,-\bar{\omega})}(-2 \bar{\omega} ; r ; \bar{\omega}) & =G_{(\omega,-\bar{\omega})}(-2 \bar{\omega} ; 1 ; \bar{\omega}) \prod_{p \mid r}\left(1-\frac{1}{p^{1-\bar{\omega}+\omega}}-\frac{1}{p^{1-2 \bar{\omega}}}+\frac{1}{p}\right)^{-1}
\end{aligned}
$$

where $G_{(\omega,-\bar{\omega})}(0 ; 1 ; \omega)=1$ and $G_{(\omega,-\bar{\omega})}(-2 \bar{\omega} ; 1 ; \bar{\omega})=1+\mathcal{O}(|\omega|)$. It follows that

$$
\begin{aligned}
& \sum_{r} \frac{\mu(r)^{2} \tau_{(\omega,-\bar{\omega})}(r) G_{(\omega,-\bar{\omega})}(0 ; r ; \omega) G_{(\omega,-\bar{\omega})}(-2 \bar{\omega} ; r ; \bar{\omega})}{r^{1+s+\omega-\bar{\omega}}} \\
& =G_{(\omega,-\bar{\omega})}(-2 \bar{\omega} ; 1 ; \bar{\omega}) \sum_{r} \frac{\mu(r)^{2} \tau_{(\omega,-\bar{\omega})}(r) \prod_{p \mid r}\left(1-\frac{1}{p^{1+\omega-\bar{\omega}}}\right)^{-1}\left(1-\frac{1}{p^{1-\bar{\omega}+\omega}}-\frac{1}{p^{1-2 \bar{\omega}}}+\frac{1}{p}\right)^{-1}}{r^{1+s+\omega-\bar{\omega}}} \\
& =G_{(\omega,-\bar{\omega})}(-2 \bar{\omega} ; 1 ; \bar{\omega}) \prod_{p}\left(1+\frac{\tau_{(\omega,-\bar{\omega})}(p)\left(1-\frac{1}{p^{1+\omega-\bar{\omega}}}\right)^{-1}\left(1-\frac{1}{p^{1-\bar{\omega}+\omega}}-\frac{1}{p^{1-2 \bar{\omega}}}+\frac{1}{p}\right)^{-1}}{p^{1+s+\omega-\bar{\omega}}}\right) \\
& =: \zeta(1+s+\omega-\bar{\omega}) H_{(\omega,-\bar{\omega})}(s),
\end{aligned}
$$

where $H_{(\omega,-\bar{\omega})}(s)$ is holomorphic for $\operatorname{Re}(s)>-1+2|\delta|$; and $H_{(\omega,-\bar{\omega})}(0)=1+\mathcal{O}(|\omega|)$. It follows from Perron's formula that the first statement in Lemma 5.28 holds. The second statement follows by the partial summation formula.

By the above lemma, we get

$$
\begin{aligned}
\Psi(\omega,-\bar{\omega}) \mathcal{V}_{(\omega,-\bar{\omega})}^{\leq}(\omega) \\
=-\frac{K}{4}\left(\frac{K}{4 \pi}\right)^{-2 \bar{\omega}} \widetilde{\Phi}(1)\left(1-M^{-(\omega-\bar{\omega})(1-\Upsilon)}\right)(1+\mathcal{O}(|\omega|)) \\
\quad \times\left(M^{-2 \bar{\omega}} \sum_{j=2}^{\infty} \frac{P^{(j)}(0)}{(-2 \bar{\omega})^{j}(\log M)^{j}}+M^{-2 \bar{\omega}(1-\Upsilon)} \sum_{j=2}^{\infty} \frac{Q^{(j)}(0)}{(-2 \bar{\omega})^{j}\left(\log M^{1-\Upsilon}\right)^{j}}\right) \\
\quad+\mathcal{O}\left(\frac{(\log \log K)^{3}}{(\log K)^{2}} K\right) .
\end{aligned}
$$

Then, by the same argument, we obtain

$$
\begin{aligned}
& \Psi(-\omega, \bar{\omega}) \mathcal{V}_{(-\omega, \bar{\omega})}^{\leq}(\omega) \\
&=-\frac{K}{4}\left(\frac{K}{4 \pi}\right)^{-2 \omega} \widetilde{\Phi}(1)\left(1-M^{-(-\omega+\bar{\omega})(1-\Upsilon)}\right)(1+\mathcal{O}(|\omega|)) \\
& \quad \times\left(M^{-2 \omega} \sum_{j=2}^{\infty} \frac{P^{(j)}(0)}{(-2 \omega)^{j}(\log M)^{j}}+M^{-2 \omega(1-\Upsilon)} \sum_{j=2}^{\infty} \frac{Q^{(j)}(0)}{(-2 \omega)^{j}\left(\log M^{1-\Upsilon}\right)^{j}}\right) \\
&+\mathcal{O}\left(\frac{(\log \log K)^{3}}{(\log K)^{2}} K\right)
\end{aligned}
$$


5.31. Contribution of the long-range terms. We first use Lemma 5.19 and equation 5.18 to deduce the estimate for $S_{(\alpha, \beta)}(r ; z)$ when $M^{1-\Upsilon}<r \leq M$.

5.32. The case $(\alpha, \beta)=(\omega, \bar{\omega})$. In this case we have

$$
S_{(\omega, \bar{\omega})}(r ; z)=\operatorname{Res}_{s=0} \frac{\mu(r) G_{(\omega, \bar{\omega})}(s ; r ; z)}{s \zeta(1+s+\omega+\bar{\omega})} \sum_{j=0}^{\infty} \frac{1}{(s \log M)^{j}} P^{(j)}\left(\frac{\log M / r}{\log M}\right)+\mathcal{O}\left(\frac{E(r)}{\log ^{2} M}\right) .
$$

Consider the Taylor expansion

$$
G_{(\omega, \bar{\omega})}(s ; r ; z) / \zeta(1+s+\omega+\bar{\omega})=a_{0}+a_{1} s+a_{2} s^{2}+a_{3} s^{3}+\ldots
$$

Then

$$
\begin{aligned}
& a_{0}=G_{(\omega, \bar{\omega})}(0 ; r ; z)(\omega+\bar{\omega})+\mathcal{O}\left(E(r)|\omega|^{2}\right), \\
& a_{1}=G_{(\omega, \bar{\omega})}(0 ; r ; z)+\mathcal{O}(E(r)|\omega|), \\
& a_{n} \ll_{n} E(r) \quad \text { for } n \geq 2 .
\end{aligned}
$$

It follows that

$$
\begin{aligned}
S_{(\omega, \bar{\omega})}(r ; z)= & \mu(r) G_{(\omega, \bar{\omega})}(0 ; r ; z)\left((\omega+\bar{\omega}) P\left(\frac{\log M / r}{\log M}\right)+\frac{1}{\log M} P^{\prime}\left(\frac{\log M / r}{\log M}\right)\right) \\
& +\mathcal{O}\left(E(r)|\omega|^{2}+\frac{E(r)}{\log ^{2} M}\right) .
\end{aligned}
$$

By (5.12), we have

$$
\begin{aligned}
\mathcal{V}_{(\omega, \bar{\omega})}^{>}(\omega)= & \sum_{M^{1-\Upsilon}<r \leq M} \frac{\mu(r)^{2} \tau_{(\omega, \bar{\omega})}(r) G_{(\omega, \bar{\omega})}(0 ; r ; \omega) G_{(\omega, \bar{\omega})}(0 ; r ; \bar{\omega})}{r^{1+\omega+\bar{\omega}}} \\
& \times\left((\omega+\bar{\omega}) P\left(\frac{\log M / r}{\log M}\right)+\frac{1}{\log M} P^{\prime}\left(\frac{\log M / r}{\log M}\right)\right)^{2} \\
& +\mathcal{O}\left(\sum_{1 \leq r \leq M}^{b} \frac{E(r)^{3}}{r}\left(|\omega|^{3}+\frac{|\omega|}{\log ^{2} K}\right)\right) .
\end{aligned}
$$

Now by Lemma 5.22, we get

$$
\begin{aligned}
\mathcal{V}_{(\omega, \bar{\omega})}^{>}(\omega)= & (1+\mathcal{O}(|\omega|)) \int_{M^{1-\Upsilon}}^{M}\left((\omega+\bar{\omega}) P\left(\frac{\log M / t}{\log M}\right)+\frac{1}{\log M} P^{\prime}\left(\frac{\log M / t}{\log M}\right)\right)^{2} \frac{d t}{t^{1+\omega+\bar{\omega}}} \\
& +\mathcal{O}\left(\frac{(\log \log K)^{3}}{\log ^{2} K}\right) .
\end{aligned}
$$

Making the change of variable $\frac{\log M / t}{\log M} \mapsto x$, we see that

$$
\begin{aligned}
\mathcal{V}_{(\omega, \bar{\omega})}^{>}(\omega)= & (\log M)(1+\mathcal{O}(|\omega|)) \int_{0}^{\Upsilon} M^{-(1-x)(\omega+\bar{\omega})}\left((\omega+\bar{\omega}) P(x)+\frac{1}{\log M} P^{\prime}(x)\right)^{2} d x \\
& +\mathcal{O}\left(\frac{(\log \log K)^{3}}{\log ^{2} K}\right) .
\end{aligned}
$$


Integrating by parts, we obtain

$$
\begin{aligned}
\mathcal{V}_{(\omega, \bar{\omega})}^{>}(\omega)= & \int_{0}^{\Upsilon}(\omega+\bar{\omega}) P(x)^{2} d M^{-(1-x)(\omega+\bar{\omega})}+2 \int_{0}^{\Upsilon} M^{-(1-x)(\omega+\bar{\omega})}(\omega+\bar{\omega}) P(x) P^{\prime}(x) d x \\
& +\frac{1}{\log M} \int_{0}^{\Upsilon} M^{-(1-x)(\omega+\bar{\omega})}\left(P^{\prime}(x)\right)^{2} d x+\mathcal{O}\left(\frac{(\log \log K)^{3}}{\log ^{2} K}\right) \\
= & \left.(\omega+\bar{\omega}) P(x)^{2} M^{-(1-x)(\omega+\bar{\omega})}\right|_{0} ^{\Upsilon} \\
& +\frac{1}{\log M} \int_{0}^{\Upsilon} M^{-(1-x)(\omega+\bar{\omega})}\left(P^{\prime}(x)\right)^{2} d x+\mathcal{O}\left(\frac{(\log \log K)^{3}}{\log ^{2} K}\right) \\
= & (\omega+\bar{\omega}) M^{-(1-\Upsilon)(\omega+\bar{\omega})}+\frac{1}{\log M} \int_{0}^{\Upsilon} M^{-(1-x)(\omega+\bar{\omega})}\left(P^{\prime}(x)\right)^{2} d x \\
& +\mathcal{O}\left(\frac{\left(\log \log ^{3}\right.}{\log ^{2} K}\right) .
\end{aligned}
$$

It then follows from 5.7 that

$$
\begin{aligned}
\Psi(\omega, \bar{\omega}) \mathcal{V}_{(\omega, \bar{\omega})}^{>}(\omega)= & \frac{K}{4} \widetilde{\Phi}(1) M^{-(1+\Upsilon)(\omega+\bar{\omega})}(1+\mathcal{O}(|\omega+\bar{\omega}|)) \\
& +\frac{K}{4} \frac{\widetilde{\Phi}(1)}{(\omega+\bar{\omega}) \log M} \int_{0}^{\Upsilon} M^{-(1-x)(\omega+\bar{\omega})}\left(P^{\prime}(x)\right)^{2} d x \\
& +\mathcal{O}\left(\frac{(\log \log K)^{4}}{\log K} K\right) .
\end{aligned}
$$

5.34. The case $(\alpha, \beta)=(-\omega,-\bar{\omega})$. In this case we have

$$
\begin{aligned}
S_{(-\omega,-\bar{\omega})}(r ; z)= & \operatorname{Res}_{s=0} \frac{\mu(r) G_{(-\omega,-\bar{\omega})}(s ; r ; z) \zeta(1+s+2 z)}{s \zeta(1+s+z-\omega) \zeta(1+s+z-\bar{\omega})} \sum_{j=0}^{\infty} \frac{1}{(s \log M)^{j}} P^{(j)}\left(\frac{\log M / r}{\log M}\right) \\
& +\frac{\mu(r) G_{(-\omega,-\bar{\omega})}(-2 z ; r ; z) r^{2 z}}{\zeta(1-z-\omega) \zeta(1-z-\bar{\omega})} M^{-2 z} \sum_{j=2}^{\infty} \frac{P^{(j)}(0)}{(-2 z)^{j+1} \log ^{j} M} \\
& +\mathcal{O}\left(\frac{E(r)}{\log ^{2} K}\right) .
\end{aligned}
$$

Consider the Taylor expansion

$$
\frac{G_{(-\omega,-\bar{\omega})}(s ; r ; z) \zeta(1+s+2 z)}{\zeta(1+s+z-\omega) \zeta(1+s+z-\bar{\omega})}=a_{0}+a_{1} s+a_{2} s^{2}+a_{3} s^{3}+\ldots
$$

Then

$$
\begin{aligned}
& a_{0}=0 \\
& a_{1}=\frac{G_{(-\omega,-\bar{\omega})}(0 ; r ; z)\left(3 z^{2}+z(-\omega-\bar{\omega})-\omega \bar{\omega}\right)}{(2 z)^{2}}+\mathcal{O}(E(r)|\omega|), \\
& a_{2}=\frac{G_{(-\omega,-\bar{\omega})}(0 ; r ; z)(z+\omega)(z+\bar{\omega})}{(2 z)^{3}}+\mathcal{O}(E(r)) \\
& a_{3}=\frac{-G_{(-\omega,-\bar{\omega})}(0 ; r ; z)(z+\omega)(z+\bar{\omega})}{(2 z)^{4}}+\mathcal{O}\left(E(r)|\omega|^{-1}\right)
\end{aligned}
$$


It follows that

$$
\begin{aligned}
& S_{(-\omega,-\bar{\omega})}(r ; z)=\mu(r) G_{(-\omega,-\bar{\omega})}(0 ; r ; z)\left(\frac{z-\bar{z}}{2 z \log M} P^{\prime}\left(\frac{\log M / r}{\log M}\right)\right. \\
& \left.+\frac{\omega+\bar{\omega}}{(2 z \log M)^{2}} P^{\prime \prime}\left(\frac{\log M / r}{\log M}\right)-\frac{\omega+\bar{\omega}}{(2 z \log M)^{3}} P^{\prime \prime \prime}\left(\frac{\log M / r}{\log M}\right)\right) \\
& +\frac{\mu(r) G_{(-\omega,-\bar{\omega})}(-2 z ; r ; z) r^{2 z}}{\zeta(1-z-\omega) \zeta(1-z-\bar{\omega})} M^{-2 z} \sum_{j=2}^{\infty} \frac{P^{(j)}(0)}{(-2 z)^{j+1} \log ^{j} M}+\mathcal{O}\left(E(r)|\omega|^{2}+\frac{E(r)}{\log ^{2} M}\right) .
\end{aligned}
$$

By 5.12 , we have

$$
\begin{aligned}
& \mathcal{V}_{(-\omega,-\bar{\omega})}^{>}(\omega) \sum_{M^{1-\Upsilon}<r \leq M} \frac{\mu(r)^{2} \tau_{(-\omega,-\bar{\omega})}(r) G_{(-\omega,-\bar{\omega})}(0 ; r ; \omega) G_{(-\omega,-\bar{\omega})}(0 ; r ; \bar{\omega})}{r^{1+\omega+\bar{\omega}}} \\
& \times\left|\frac{\omega-\bar{\omega}}{2 \omega \log M} P^{\prime}\left(\frac{\log M / r}{\log M}\right)+\frac{\omega+\bar{\omega}}{(2 \omega \log M)^{2}} P^{\prime \prime}\left(\frac{\log M / r}{\log M}\right)-\frac{\omega+\bar{\omega}}{(2 \omega \log M)^{3}} P^{\prime \prime \prime}\left(\frac{\log M / r}{\log M}\right)\right|^{2} \\
&+2 \operatorname{Re} \sum_{M^{1-\Upsilon}<r \leq M} \frac{\mu(r)^{2} \tau_{(-\omega,-\bar{\omega})}(r) G_{(-\omega,-\bar{\omega})}(0 ; r ; \omega) G_{(-\omega,-\bar{\omega})}(-2 \bar{\omega} ; r ; \bar{\omega})}{r^{1+\omega-\bar{\omega}} \zeta(1-\omega-\bar{\omega}) \zeta(1-2 \bar{\omega})} \\
& \times\left(M^{-2 \bar{\omega}} \sum_{j=2}^{\infty} \frac{P^{(j)}(0)}{(-2 \bar{\omega})^{j+1} \log ^{j} M}\right)\left(\frac{\omega-\bar{\omega}}{2 \omega \log M} P^{\prime}\left(\frac{\log M / r}{\log M}\right)\right. \\
&+\left.\frac{\omega+\bar{\omega}}{(2 \omega \log M)^{2}} P^{\prime \prime}\left(\frac{\log M / r}{\log M}\right)-\frac{\omega+\bar{\omega}}{(2 \omega \log M)^{3}} P^{\prime \prime \prime}\left(\frac{\log M / r}{\log M}\right)\right) \\
&+\quad \sum_{M^{1-\Upsilon}<r \leq M} \frac{\mu(r)^{2} \tau_{(-\omega,-\bar{\omega})}(r) G_{(-\omega,-\bar{\omega})}(-2 \omega ; r ; \omega) G_{(-\omega,-\bar{\omega})}(-2 \bar{\omega} ; r ; \bar{\omega})}{r^{1-\omega-\bar{\omega}} \zeta(1-2 \omega) \zeta(1-\omega-\bar{\omega})^{2} \zeta(1-2 \bar{\omega})} \\
& \quad \times\left|M^{-2 \omega} \sum_{j=2}^{\infty} \frac{P^{(j)}(0)}{(-2 \omega)^{j+1} \log ^{j} M}\right|^{2}+\mathcal{O}\left(\frac{(\log \log K)^{3}}{\log { }^{2} K}\right) .
\end{aligned}
$$

Next, Lemma 5.25 implies that

$$
\begin{aligned}
\mathcal{V}_{(-\omega,-\bar{\omega})}^{>}(\omega)= & (1+\mathcal{O}(|\omega|)) \int_{M^{1-\Upsilon}}^{M} \mid \frac{\omega-\bar{\omega}}{2 \omega \log M} P^{\prime}\left(\frac{\log M / t}{\log M}\right) \\
& +\frac{\omega+\bar{\omega}}{(2 \omega \log M)^{2}} P^{\prime \prime}\left(\frac{\log M / t}{\log M}\right)-\left.\frac{\omega+\bar{\omega}}{(2 \omega \log M)^{3}} P^{\prime \prime \prime}\left(\frac{\log M / t}{\log M}\right)\right|^{2} \frac{d t}{t^{1+\omega+\bar{\omega}}} \\
& +2 \operatorname{Re}\left\{\frac{1+\mathcal{O}(|\omega|)}{\zeta(1-\omega-\bar{\omega})}\left(M^{-2 \bar{\omega}} \sum_{j=2}^{\infty} \frac{P^{(j)}(0)}{(-2 \bar{\omega} \log M)^{j}}\right)\right. \\
& \times \int_{M^{1-\Upsilon}}^{M}\left(\frac{\omega-\bar{\omega}}{2 \omega \log M} P^{\prime}\left(\frac{\log M / t}{\log M}\right)+\frac{\omega+\bar{\omega}}{(2 \omega \log M)^{2}} P^{\prime \prime}\left(\frac{\log M / t}{\log M}\right)\right. \\
& \left.\left.-\frac{\omega+\bar{\omega}}{(2 \omega \log M)^{3}} P^{\prime \prime \prime}\left(\frac{\log M / t}{\log M}\right)\right) \frac{d t}{t^{1+\omega-\bar{\omega}}}\right\}
\end{aligned}
$$




$$
\begin{aligned}
& +\frac{1+\mathcal{O}(|\omega|)}{\zeta(1-\omega-\bar{\omega})}\left(M^{(1-\Upsilon)(\omega+\bar{\omega})}-M^{\omega+\bar{\omega}}\right) \cdot\left|M^{-2 \omega} \sum_{j=2}^{\infty} \frac{P^{(j)}(0)}{(-2 \omega \log M)^{j}}\right|^{2} \\
& +\mathcal{O}\left(\frac{(\log \log K)^{3}}{\log ^{2} K}\right) .
\end{aligned}
$$

Making the change of variable $\frac{\log M / t}{\log M} \mapsto x$, we see that

$$
\begin{aligned}
& \mathcal{V}_{(-\omega,-\bar{\omega})}^{>}(\omega)=\frac{1+\mathcal{O}(|\omega|)}{\log M} \\
& \times \int_{0}^{\Upsilon} M^{-(1-x)(\omega+\bar{\omega})}\left|\frac{\omega-\bar{\omega}}{2 \omega} P^{\prime}(x)+\frac{\omega+\bar{\omega}}{(2 \omega)^{2} \log M} P^{\prime \prime}(x)-\frac{\omega+\bar{\omega}}{(2 \omega)^{3}(\log M)^{2}} P^{\prime \prime \prime}(x)\right|^{2} d x \\
& +2 \operatorname{Re}\left\{\frac{1+\mathcal{O}(|\omega|)}{\zeta(1-\omega-\bar{\omega})}\left(M^{-2 \bar{\omega}} \sum_{j=2}^{\infty} \frac{P^{(j)}(0)}{(-2 \bar{\omega} \log M)^{j}}\right) \int_{0}^{\Upsilon} M^{-(1-x)(\omega-\bar{\omega})}\right. \\
& \left.\times\left(\frac{\omega-\bar{\omega}}{2 \omega} P^{\prime}(x)+\frac{\omega+\bar{\omega}}{(2 \omega)^{2} \log M} P^{\prime \prime}(x)-\frac{\omega+\bar{\omega}}{(2 \omega)^{3}(\log M)^{2}} P^{\prime \prime \prime}(x)\right) d x\right\} \\
& +\frac{1+\mathcal{O}(|\omega|)}{\zeta(1-\omega-\bar{\omega})}\left(M^{(1-\Upsilon)(\omega+\bar{\omega})}-M^{\omega+\bar{\omega}}\right) \cdot\left|M^{-2 \omega} \sum_{j=2}^{\infty} \frac{P^{(j)}(0)}{(-2 \omega \log M)^{j}}\right|^{2} \\
& +\mathcal{O}\left(\frac{(\log \log K)^{3}}{\log ^{2} K}\right) .
\end{aligned}
$$

Hence by (5.7), we obtain

$$
\begin{aligned}
\Psi & (-\omega,-\bar{\omega}) \mathcal{V}_{(-\omega,-\bar{\omega})}^{>}(\omega) \\
= & \frac{K}{4}\left(\frac{K}{4 \pi}\right)^{-2(\omega+\bar{\omega})} \widetilde{\Phi}(1)(1+\mathcal{O}(|\omega|))\left[\frac{-1}{(\omega+\bar{\omega}) \log M} \int_{0}^{\Upsilon} M^{-(1-x)(\omega+\bar{\omega})}\right. \\
& \times\left|\frac{\omega-\bar{\omega}}{2 \omega} P^{\prime}(x)+\frac{\omega+\bar{\omega}}{(2 \omega)^{2} \log M} P^{\prime \prime}(x)-\frac{\omega+\bar{\omega}}{(2 \omega)^{3}(\log M)^{2}} P^{\prime \prime \prime}(x)\right|^{2} d x \\
& +2 \operatorname{Re}\left\{M^{-2 \bar{\omega}} \sum_{j=2}^{\infty} \frac{P^{(j)}(0)}{(-2 \bar{\omega} \log M)^{j}} \int_{0}^{\Upsilon} M^{-(1-x)(\omega-\bar{\omega})}\right. \\
& \left.\times\left(\frac{\omega-\bar{\omega}}{2 \omega} P^{\prime}(x)+\frac{\omega+\bar{\omega}}{(2 \omega)^{2} \log M} P^{\prime \prime}(x)-\frac{\omega+\bar{\omega}}{(2 \omega)^{3}(\log M)^{2}} P^{\prime \prime \prime}(x)\right) d x\right\} \\
& \left.+\left(M^{(1-\Upsilon)(\omega+\bar{\omega})}-M^{\omega+\bar{\omega}}\right)\left|M^{-2 \omega} \sum_{j=2}^{\infty} \frac{P^{(j)}(0)}{(-2 \omega \log M)^{j}}\right|^{2}\right] \\
& +\mathcal{O}\left(\frac{(\log \log K)^{4}}{\log K} K^{2}\right) .
\end{aligned}
$$


5.36. The cases $(\alpha, \beta)=(\omega,-\bar{\omega})$ and $(\alpha, \beta)=(-\omega, \bar{\omega})$. We first consider the case $(\alpha, \beta)=(\omega,-\bar{\omega})$. By Lemma 5.19 , we have

$$
\begin{aligned}
S_{(\omega,-\bar{\omega})}(r ; \omega)= & \operatorname{Res}_{s=0} \frac{\mu(r) G_{(\omega,-\bar{\omega})}(s ; r ; \omega)}{s \zeta(1+s+\omega-\bar{\omega})} \sum_{j=0}^{\infty} \frac{1}{(s \log M)^{j}} P^{(j)}\left(\frac{\log M / r}{\log M}\right) \\
& +\mathcal{O}\left(\frac{E(r)}{\log ^{2} K}\right),
\end{aligned}
$$

and

$$
\begin{aligned}
S_{(\omega,-\bar{\omega})}(r ; \bar{\omega})= & \operatorname{Res}_{s=0} \frac{\mu(r) G_{(\omega,-\bar{\omega})}(s ; r ; \bar{\omega}) \zeta(1+s+2 \bar{\omega})}{s \zeta(1+s+\omega+\bar{\omega}) \zeta(1+s)} \sum_{j=0}^{\infty} \frac{1}{(s \log M)^{j}} P^{(j)}\left(\frac{\log M / r}{\log M}\right) \\
& +\frac{\mu(r) G_{(\omega,-\bar{\omega})}(-2 \bar{\omega} ; r ; \bar{\omega}) r^{2 \bar{\omega}}}{\zeta(1+\omega-\bar{\omega}) \zeta(1-2 \bar{\omega})} M^{-2 \bar{\omega}} \sum_{j=2}^{\infty} \frac{P^{(j)}(0)}{(-2 \bar{\omega})^{j+1} \log ^{j} M} \\
& +\mathcal{O}\left(\frac{E(r)}{\log ^{2} K}\right) .
\end{aligned}
$$

We first write the Taylor expansion of $G_{(\omega,-\bar{\omega})}(s ; r ; \omega) / \zeta(1+s+\omega-\bar{\omega})$ as $a_{0}+a_{1} s+$ $a_{2} s^{2}+a_{3} s^{3}+\ldots$ Then

$$
\begin{aligned}
& a_{0}=G_{(\omega,-\bar{\omega})}(0 ; r ; \omega)(\omega-\bar{\omega})+\mathcal{O}\left(E(r)|\omega|^{2}\right), \\
& a_{1}=G_{(\omega,-\bar{\omega})}(0 ; r ; \omega)+\mathcal{O}(E(r)|\omega|), \\
& a_{n} \ll_{n} E(r), \quad \text { if } n \geq 2 .
\end{aligned}
$$

Next, consider the Taylor expansion

$$
\frac{G_{(\omega,-\bar{\omega})}(s ; r ; \bar{\omega}) \zeta(1+s+2 \bar{\omega})}{\zeta(1+s+\omega+\bar{\omega}) \zeta(1+s)}=b_{0}+b_{1} s+b_{2} s^{2}+b_{3} s^{3}+\ldots
$$

Then we have

$$
\begin{aligned}
& b_{0}=0, \\
& b_{1}=\frac{G_{(\omega,-\bar{\omega})}(0 ; r ; \bar{\omega})(\omega+\bar{\omega})}{2 \bar{\omega}}+\mathcal{O}(E(r)|\omega|), \\
& b_{2}=\frac{G_{(\omega,-\bar{\omega})}(0 ; r ; \bar{\omega})(\bar{\omega}-\omega)}{(2 \bar{\omega})^{2}}+\mathcal{O}(E(r)), \\
& b_{3}=\frac{-G_{(\omega,-\bar{\omega})}(0 ; r ; \bar{\omega})(\bar{\omega}-\omega)}{(2 \bar{\omega})^{3}}+\mathcal{O}\left(E(r)|\omega|^{-1}\right) .
\end{aligned}
$$

So we get

$$
\begin{aligned}
S_{(\omega,-\bar{\omega})}(r ; \omega)= & \mu(r) G_{(\omega,-\bar{\omega})}(0 ; r ; \omega)\left((\omega-\bar{\omega}) P\left(\frac{\log M / r}{\log M}\right)+\frac{1}{\log M} P^{\prime}\left(\frac{\log M / r}{\log M}\right)\right) \\
& +\mathcal{O}\left(\frac{\log \log K}{\log ^{2} K}\right)
\end{aligned}
$$


and

$$
\begin{aligned}
S_{(\omega,-\bar{\omega})}(r ; \bar{\omega})= & \mu(r) G_{(\omega,-\bar{\omega})}(0 ; r ; \bar{\omega})\left(\frac{\omega+\bar{\omega}}{2 \bar{\omega} \log M} P^{\prime}\left(\frac{\log M / r}{\log M}\right)\right. \\
& \left.+\frac{\bar{\omega}-\omega}{(2 \bar{\omega} \log M)^{2}} P^{\prime \prime}\left(\frac{\log M / r}{\log M}\right)+\frac{\omega-\bar{\omega}}{(2 \bar{\omega} \log M)^{3}} P^{\prime \prime \prime}\left(\frac{\log M / r}{\log M}\right)\right) \\
& +\frac{\mu(r) G_{(\omega,-\bar{\omega})}(-2 \bar{\omega} ; r ; \bar{\omega}) r^{2 \bar{\omega}}}{\zeta(1+\omega-\bar{\omega})} M^{-2 \bar{\omega}} \sum_{j=2}^{\infty} \frac{P^{(j)}(0)}{(-2 \bar{\omega} \log M)^{j}} \\
& +\mathcal{O}\left(\frac{\log \log K}{\log ^{2} K}\right) .
\end{aligned}
$$

By 5.12 , we have

$$
\begin{aligned}
& \mathcal{V}_{(\omega,-\bar{\omega})}^{>}(\omega)=\sum_{M^{1-\Upsilon}<r \leq M} \frac{\mu(r)^{2} \tau_{(\omega,-\bar{\omega})}(r) G_{(\omega,-\bar{\omega})}(0 ; r ; \omega) G_{(\omega,-\bar{\omega})}(0 ; r ; \bar{\omega})}{r^{1+\omega+\bar{\omega}}} \\
& \times\left(\left((\omega-\bar{\omega}) P\left(\frac{\log M / r}{\log M}\right)+\frac{1}{\log M} P^{\prime}\left(\frac{\log M / r}{\log M}\right)\right)\right. \\
& \times\left(\frac{\omega+\bar{\omega}}{2 \bar{\omega} \log M} P^{\prime}\left(\frac{\log M / r}{\log M}\right)+\frac{\bar{\omega}-\omega}{(2 \bar{\omega} \log M)^{2}} P^{\prime \prime}\left(\frac{\log M / r}{\log M}\right)+\frac{\omega-\bar{\omega}}{(2 \bar{\omega} \log M)^{3}} P^{\prime \prime \prime}\left(\frac{\log M / r}{\log M}\right)\right) \\
& +\sum_{M^{1-\Upsilon}<r \leq M} \frac{\mu(r)^{2} \tau_{(\omega,-\bar{\omega})}(r) G_{(\omega,-\bar{\omega})}(0 ; r ; \omega) G_{(\omega,-\bar{\omega})}(-2 \bar{\omega} ; r ; \bar{\omega})}{r^{1+\omega-\bar{\omega}} \zeta(1+\omega-\bar{\omega})} \\
& \times\left(M^{-2 \bar{\omega}} \sum_{j=2}^{\infty} \frac{P^{(j)}(0)}{(-2 \bar{\omega} \log M)^{j}}\right)\left((\omega-\bar{\omega}) P\left(\frac{\log M / r}{\log M}\right)+\frac{1}{\log M} P^{\prime}\left(\frac{\log M / r}{\log M}\right)\right) \\
& +\mathcal{O}\left(\frac{(\log \log K)^{3}}{\log ^{2} K}\right) \text {. }
\end{aligned}
$$

Applying Lemma 5.25, with the change of variables from $\frac{\log M / t}{\log M}$ to $x$, together with (5.7), we see that

$$
\begin{aligned}
& \Psi(\omega,-\bar{\omega}) \mathcal{V}_{(\omega,-\bar{\omega})}^{>}(\omega) \\
= & -\frac{K}{4}\left(\frac{K}{4 \pi}\right)^{-2 \bar{\omega}} \widetilde{\Phi}(1)(1+\mathcal{O}(|\omega|)) \\
\times & {\left[\frac{1}{(\omega-\bar{\omega}) \log M} \int_{0}^{\Upsilon} M^{-(1-x)(\omega+\bar{\omega})}\left((\omega-\bar{\omega})(\log M) P(x)+P^{\prime}(x)\right)\right.} \\
\times & \left(\frac{\omega+\bar{\omega}}{2 \bar{\omega}} P^{\prime}(x)+\frac{\bar{\omega}-\omega}{(2 \bar{\omega})^{2} \log M} P^{\prime \prime}(x)+\frac{\omega-\bar{\omega}}{(2 \bar{\omega})^{3}(\log M)^{2}} P^{\prime \prime \prime}(x)\right) d x \\
+ & \left.M^{-2 \bar{\omega}} \sum_{j=2}^{\infty} \frac{P^{(j)}(0)}{(-2 \bar{\omega} \log M)^{j}} \int_{0}^{\Upsilon} M^{-(1-x)(\omega-\bar{\omega})} \cdot\left((\omega-\bar{\omega})(\log M) P(x)+P^{\prime}(x)\right) d x\right] \\
+ & \mathcal{O}\left(\frac{(\log \log K)^{4}}{\log K} K\right) .
\end{aligned}
$$


By the same argument we also obtain

$$
\begin{aligned}
& =-\frac{K}{4}\left(\frac{K}{4 \pi}\right)^{-2 \omega} \widetilde{\Phi}(1)(1+\mathcal{O}(|\omega|)) \\
& \times\left[\frac{1}{(-\omega+\bar{\omega}) \log M} \int_{0}^{\Upsilon} M^{-(1-x)(\omega+\bar{\omega})}\left((-\omega+\bar{\omega})(\log M) P(x)+P^{\prime}(x)\right)\right. \\
& \times\left(\frac{\omega+\bar{\omega}}{2 \bar{\omega}} P^{\prime}(x)+\frac{\omega-\bar{\omega}}{(2 \bar{\omega})^{2} \log M} P^{\prime \prime}(x)-\frac{\omega-\bar{\omega}}{(2 \bar{\omega})^{3}(\log M)^{2}} P^{\prime \prime \prime}(x)\right) d x \\
& \left.+M^{-2 \omega} \sum_{j=2}^{\infty} \frac{P^{(j)}(0)}{(-2 \omega \log M)^{j}} \int_{0}^{\Upsilon} M^{-(1-x)(-\omega+\bar{\omega})} \cdot\left((-\omega+\bar{\omega})(\log M) P(x)+P^{\prime}(x)\right) d x\right] \\
& +\mathcal{O}\left(\frac{(\log \log K)^{4}}{\log K} K\right) \text {. }
\end{aligned}
$$

5.39. Conclusion. Recall the harmonic weight

$$
\omega_{f}:=\frac{12 \zeta(2)}{(k-1)} \cdot \frac{1}{L\left(1, \operatorname{sym}^{2} f\right)} .
$$

Now $\Phi$ is a non-negative smooth function supported on $[1,2]$ such that $\Phi(t) \ll 1$, and $\int_{1}^{2} \Phi(t) d t \gg 1$. It follows from 3.10 that

$$
\begin{aligned}
\mathcal{A}(K, \Phi) & =\sum_{k \equiv 2(4)} \Phi\left(\frac{k-1}{K}\right) \sum_{f \in H_{k}} \omega_{f} \\
& =\sum_{k \equiv 2(4)} \Phi\left(\frac{k-1}{K}\right)\left(1+\mathcal{O}\left(2^{-k}\right)\right)=\frac{K}{4} \widetilde{\Phi}(1)+\mathcal{O}\left(K^{-B}\right) .
\end{aligned}
$$

Combine 5.6 and $5.23-5.38$ and make the change of variables $\delta=\frac{u}{\log K}, t=\frac{v}{\log K}$. If we then take $M=K^{1-5 \vartheta}$, it follows that

$$
\frac{1}{\mathcal{A}(K, \Phi)} \mathcal{A}\left(\left\{|L M(1 / 2+\omega, f)|^{2}\right\} ; K, \Phi\right)=\mathscr{V}(u, v)+\mathcal{O}\left(\frac{(\log \log K)^{4}}{\log K}\right) .
$$

Here

$$
\begin{aligned}
\mathscr{V}_{1}(u, v):= & 1+\frac{1}{2 u(1-5 \vartheta)} \int_{0}^{\Upsilon} e^{-2 u(1-x)(1-5 \vartheta)}\left(P^{\prime}(x)\right)^{2} d x \\
\mathscr{V}_{2}(u, v):= & e^{-4 u}\left[\frac{-1}{2 u(1-5 \vartheta)} \int_{0}^{\Upsilon} e^{-2 u(1-x)(1-5 \vartheta)}\right. \\
& \times\left|\frac{i v}{u+i v} P^{\prime}(x)+\frac{u}{2(u+i v)^{2}(1-5 \vartheta)} P^{\prime \prime}(x)-\frac{u}{4(u+i v)^{3}(1-5 \vartheta)^{2}} P^{\prime \prime \prime}(x)\right|^{2} d x \\
& +2 \operatorname{Re}\left\{e^{-2(1-5 \vartheta)(u-i v)} \sum_{j=2}^{\infty} \frac{P^{(j)}(0)}{(-2(u-i v)(1-5 \vartheta))^{j}}\right.
\end{aligned}
$$




$$
\begin{aligned}
& \times \int_{0}^{\Upsilon} e^{-2 i v(1-x)(1-5 \vartheta)}\left(\frac{i v}{u+i v} P^{\prime}(x)+\frac{u}{2(u+i v)^{2}(1-5 \vartheta)} P^{\prime \prime}(x)\right. \\
& \left.\left.-\frac{u}{4(u+i v)^{3}(1-5 \vartheta)^{2}} P^{\prime \prime \prime}(x)\right) d x\right\} \\
& +\left(e^{2 u(1-5 \vartheta)(1-\Upsilon)}-e^{2 u(1-5 \vartheta)}\right)\left|e^{-2(u+i v)(1-5 \vartheta)} \sum_{j=2}^{\infty} \frac{P^{(j)}(0)}{(-2(u+i v)(1-5 \vartheta))^{j}}\right|^{2} \\
& +\left(1-e^{2 u(1-5 \vartheta)(1-\Upsilon)}\right) \mid e^{-2(u+i v)(1-5 \vartheta)} \sum_{j=2}^{\infty} \frac{P^{(j)}(0)}{(-2(u+i v)(1-5 \vartheta))^{j}} \\
& \left.+\left.e^{-2(u+i v)(1-5 \vartheta)(1-\Upsilon)} \sum_{j=2}^{\infty} \frac{Q^{(j)}(0)}{(-2(u+i v)(1-5 \vartheta)(1-\Upsilon))^{j}}\right|^{2}\right], \\
& \mathscr{V}_{3}(u, v):=-2 \operatorname{Re}\left\{e ^ { - 2 ( u + i v ) } \left[\frac{1}{-2 i v(1-5 \vartheta)}\right.\right. \\
& \times \int_{0}^{\Upsilon} e^{-2 u(1-x)(1-5 \vartheta)}\left(-2 i v(1-5 \vartheta) P(x)+P^{\prime}(x)\right) \\
& \times\left(\frac{u}{(u+i v)} P^{\prime}(x)+\frac{i v}{2(u+i v)^{2}(1-5 \vartheta)} P^{\prime \prime}(x)\right. \\
& \left.-\frac{i v}{4(u+i v)^{3}(1-5 \vartheta)^{2}} P^{\prime \prime \prime}(x)\right) d x+e^{-2(u+i v)(1-5 \vartheta)} \sum_{j=2}^{\infty} \frac{P^{(j)}(0)}{(-2(u+i v)(1-5 \vartheta))^{j}} \\
& \times \int_{0}^{\Upsilon} e^{2 i v(1-x)(1-5 \vartheta)}\left(-2 i v(1-5 \vartheta) P(x)+P^{\prime}(x)\right) d x \\
& +\left(1-e^{2 i v(1-\Upsilon)(1-5 \vartheta)}\right)\left(e^{-2(u+i v)(1-5 \vartheta)} \sum_{j=2}^{\infty} \frac{P^{(j)}(0)}{(-2(u+i v)(1-5 \vartheta))^{j}}\right. \\
& \left.\left.\left.+e^{-2(u+i v)(1-5 \vartheta)(1-\Upsilon)} \sum_{j=2}^{\infty} \frac{Q^{(j)}(0)}{\left(-2(u+i v)(1-5 \vartheta)(1-\Upsilon)^{j}\right.}\right)\right]\right\} .
\end{aligned}
$$

We end this section by proving the following upper bound for $\mathscr{V}(u, v)$, which will be used when $u$ is large.

5.43. Lemma. Choose

$$
\begin{gathered}
P(x)=3\left(\frac{x}{\Upsilon}\right)^{2}-2\left(\frac{x}{\Upsilon}\right)^{3}, \quad Q(x):=1-P(\Upsilon+(1-\Upsilon) x), \\
\vartheta=10^{-10}, \quad \Upsilon=0.64, \quad S=\frac{\pi}{4(1-\Upsilon)(1-20 \vartheta)}, \quad R=4 .
\end{gathered}
$$

For $u \geq 10$ and $|v| \leq 5 u$, we have

$$
\mathscr{V}(u, v) \leq 1+e^{-u / 2} .
$$

Proof. To prove the lemma, we will show that for $u \geq 10$ and $|v| \leq 5 u$,

$$
\mathscr{V}_{1}(u, v) \leq 1+\frac{1}{2} e^{-u / 2}, \quad \mathscr{V}_{2}(u, v) \leq e^{-4 u}, \quad \mathscr{V}_{3}(u, v) \leq e^{-2 u} .
$$


In fact, much better bounds can be proved, but this will be good enough for our applications.

Recall the definition of $\mathscr{V}_{j}(u, v)$ above. Using the fact that $\left|P^{\prime}(x)\right| \leq 3 /(2 \Upsilon)$ if $x \in[0, \Upsilon]$, one may easily obtain the first inequality in (5.44). Indeed, we have

$$
1 \leq \mathscr{V}_{1}(u, v) \leq 1+\frac{\Upsilon}{2 u(1-5 \vartheta)} e^{-2 u(1-\Upsilon)(1-5 \vartheta)}\left(\frac{3}{2 \Upsilon}\right)^{2} \leq 1+\frac{1}{2} e^{-u / 2},
$$

for $u \geq 10$. Next we will consider $\mathscr{V}_{2}(u, v)$. For $x \in[0, \Upsilon]$

Hence

$$
\left|P^{\prime \prime}(x)\right| \leq \frac{6}{\Upsilon^{2}}, \quad P^{\prime \prime \prime}(x)=\frac{12}{\Upsilon^{3}} .
$$

$$
\begin{aligned}
\left|\mathscr{V}_{2}(u, v)\right| \leq & e^{-4 u}\left\{\frac{\Upsilon}{2 u(1-5 \vartheta)} e^{-2 u(1-x)(1-5 \vartheta)}\left(\left(\frac{3}{2 \Upsilon}\right)^{2}+\left(\frac{6}{u \Upsilon^{2}}\right)^{2}+\left(\frac{12}{u^{2} \Upsilon^{3}}\right)^{2}\right)\right. \\
& +2\left(e^{-2 u(1-5 \vartheta)}\left(\frac{6}{u^{2} \Upsilon^{2}}+\frac{12}{u^{3} \Upsilon^{3}}\right) \Upsilon\left(\frac{3}{2 \Upsilon}+\frac{6}{u \Upsilon^{2}}+\frac{12}{u^{2} \Upsilon^{3}}\right)\right) \\
& \left.+6 e^{-2 u(1-5 \vartheta)(1-\Upsilon)}\left(\left(\frac{6}{u^{2} \Upsilon^{2}}\right)^{2}+\left(\frac{12}{u^{3} \Upsilon^{3}}\right)^{2}\right)\right\} .
\end{aligned}
$$

For $u \geq 10$, we have $\left|\mathscr{V}_{2}(u, v)\right| \leq e^{-4 u}$, which gives the second inequality in (5.44). Finally, we will bound $\mathscr{V}_{3}(u, v)$. Note that

$$
\mathscr{V}_{3}(u, v)=\mathscr{V}_{31}(u, v)+\mathscr{V}_{32}(u, v),
$$

where

$$
\begin{aligned}
\mathscr{V}_{31}(u, v):= & -2 \operatorname{Re}\left\{e^{-2(u+i v)} \frac{u}{-2 i v(u+i v)(1-5 \vartheta)} \int_{0}^{\Upsilon} e^{-2 u(1-x)(1-5 \vartheta)}\left(P^{\prime}(x)\right)^{2} d x\right\}, \\
\mathscr{V}_{32}(u, v):= & -2 \operatorname{Re}\left\{e ^ { - 2 ( u + i v ) } \left[\int _ { 0 } ^ { \Upsilon } e ^ { - 2 u ( 1 - x ) ( 1 - 5 \vartheta ) } P ( x ) \left(\frac{u}{(u+i v)} P^{\prime}(x)\right.\right.\right. \\
& \left.+\frac{i v}{2(u+i v)^{2}(1-5 \vartheta)} P^{\prime \prime}(x)-\frac{i v}{4(u+i v)^{3}(1-5 \vartheta)^{2}} P^{\prime \prime \prime}(x)\right) d x \\
& -\frac{1}{2(1-5 \vartheta)} \int_{0}^{\Upsilon} e^{-2 u(1-x)(1-5 \vartheta)} P^{\prime}(x) \\
& \times\left(\frac{1}{2(u+i v)^{2}(1-5 \vartheta)} P^{\prime \prime}(x)-\frac{1}{4(u+i v)^{3}(1-5 \vartheta)^{2}} P^{\prime \prime \prime}(x)\right) d x \\
& +e^{-2(u+i v)(1-5 \vartheta)} \sum_{j=2}^{\infty} \frac{P^{(j)}(0)}{(-2(u+i v)(1-5 \vartheta))^{j}} \\
& \times \int_{0}^{\Upsilon} e^{2 i v(1-x)(1-5 \vartheta)}\left(-2 i v(1-5 \vartheta) P(x)+P^{\prime}(x)\right) d x \\
& +\left(1-e^{2 i v(1-\Upsilon)(1-5 \vartheta)}\right)\left(e^{-2(u+i v)(1-5 \vartheta)} \sum_{j=2}^{\infty} \frac{P^{(j)}(0)}{(-2(u+i v)(1-5 \vartheta))^{j}}\right. \\
& \left.\left.\left.+e^{-2(u+i v)(1-5 \vartheta)(1-\Upsilon)} \sum_{j=2}^{\infty} \frac{Q^{(j)}(0)}{(-2(u+i v)(1-5 \vartheta)(1-\Upsilon))^{j}}\right)\right]\right\} . \\
& (1-2(u)
\end{aligned}
$$


By the same argument as above we can show that $\left|\mathscr{V}_{32}(u, v)\right| \leq \frac{1}{2} e^{-2 u}$. We also have

$$
\left|\mathscr{V}_{31}(u, v)\right| \leq \frac{2 u e^{-2 u}}{(1-5 \vartheta)}\left(\int_{0}^{\Upsilon} e^{-2 u(1-x)(1-5 \vartheta)}\left(P^{\prime}(x)\right)^{2} d x\right)\left|\operatorname{Re}\left\{\frac{e^{-2 i v}}{-2 i v(u+i v)}\right\}\right| \leq \frac{1}{2} e^{-2 u} .
$$

This establishes the third inequality in 5.44 and completes the proof of the lemma.

6. The harmonic mollified second moment away from the critical point. In this section, we get a bound for $\frac{1}{\mathcal{A}(K, \Phi)} \mathcal{A}\left(\left\{|L M(1 / 2+\delta+i t, f)|^{2}\right\} ; K, \Phi\right)$ when

$$
\frac{\log \log K}{\log K} \ll \delta \leq 1 / 2+\frac{10 \log \log K}{(1-\Upsilon) \log K} .
$$

We will follow the method of Ricotta [18, Appendix A], which is based on a classical Phragmén-Lindelöf-type convexity principle.

6.1. TheOREM. Let $0<\Upsilon<1$ and $M=K^{1-5 \vartheta}$, with $0<\vartheta<1 / 100$ being a small constant. If $\delta \gg \frac{\log \log K}{\log K}$, then for any $0<a<2(1-\Upsilon)$ we have

$$
\frac{1}{\mathcal{A}(K, \Phi)} \mathcal{A}\left(\left\{|L M(1 / 2+\delta+i t, f)-1|^{2}\right\} ; K, \Phi\right) \ll_{B, a}(1+|t|)^{B} M^{-a \delta},
$$

for some constant $B>0$ depending only on $\vartheta$. If $\frac{\log \log K}{\log K} \ll \delta \leq 1 / 2+\frac{10 \log \log K}{(1-\Upsilon) \log K}$, then for any $0<a<2(1-\Upsilon)$,

$$
\frac{\mathcal{A}\left(\left\{|L M(1 / 2+\delta+i t, f)|^{2}\right\} ; K, \Phi\right)}{\mathcal{A}(K, \Phi)}=1+\mathcal{O}_{B, a}\left((1+|t|)^{B} M^{-a \delta}\right),
$$

for some constant $B>0$ depending only on $\vartheta$.

The proof of Theorem 6.1 (to be given at the end of this section) requires the following three lemmas.

6.2. Lemma. Let $\vartheta, \Upsilon, M$ be as in Theorem 6.1. If $\delta=\frac{\log \log \log K}{\log K}$, then

$$
\frac{\mathcal{A}\left(\left\{|L M(1 / 2+\delta+i t, f)|^{2}\right\} ; K, \Phi\right)}{\mathcal{A}(K, \Phi)} \ll_{B}(1+|t|)^{B},
$$

for some constant $B>0$ depending only on $\vartheta$.

Proof. If $|t| \ll \frac{\log \log K}{\log K}$, then by the argument in $\$ 4$ and $\$ 5$ it follows that

$$
\frac{\mathcal{A}\left(\left\{|L M(1 / 2+\delta+i t, f)|^{2}\right\} ; K, \Phi\right)}{\mathcal{A}(K, \Phi)} \ll 1,
$$

(see 5.41$),(5.42)$. So we may assume $|t| \gg \frac{\log \log K}{\log K}$. Let $\theta=\vartheta / 2$. If $|t| \gg K^{\theta}$, then one can use the convexity bounds for $L(1 / 2+\delta+i t, f)$ and $M(1 / 2+\delta+i t, f)$ to deduce our claim, provided that $B>0$ is large enough. Consequently, we only need to handle the case $\frac{\log \log K}{\log K} \ll|t| \ll K^{\theta}$. As in $\$ 5$, by Theorem 4.2 , we have

$$
\begin{gathered}
\mathcal{A}\left(\left\{|L M(1 / 2+\delta+i t, f)|^{2}\right\} ; K, \Phi\right) \\
=\sum_{d}^{b} \frac{1}{d^{1+2 \delta}} \sum_{\begin{array}{c}
\left(m_{1}, n_{1}\right)=1 \\
\left(m_{2}, n_{2}\right)=1 \\
\left(m_{1} n_{1} m_{2} n_{2}, d\right)=1
\end{array}}^{b} \frac{\mu\left(m_{1}\right) \mu\left(m_{2}\right) F_{\Upsilon, M}\left(d m_{1} n_{1}\right) F_{\Upsilon, M}\left(d m_{2} n_{2}\right)}{\left(m_{1} n_{1}^{2}\right)^{1 / 2+\delta+i t}\left(m_{2} n_{2}^{2}\right)^{1 / 2+\delta-i t}}
\end{gathered}
$$




$$
\begin{aligned}
& \times\left[\zeta(1+2 \delta) \frac{\eta_{i t}\left(m_{1} m_{2}\right)}{\left(m_{1} m_{2}\right)^{1 / 2+\delta}} \frac{K}{4} \int_{0}^{\infty} \Phi(u) d u\right. \\
& +\zeta(1-2 \delta) \frac{\eta_{i t}\left(m_{1} m_{2}\right)}{\left(m_{1} m_{2}\right)^{1 / 2-\delta}}\left(\frac{K}{4 \pi}\right)^{-4 \delta} \frac{K}{4} \int_{0}^{\infty} \Phi(u) u^{-4 \delta} d u \\
& \left.-2 \operatorname{Re}\left\{\zeta(1-2 i t) \frac{\eta_{\delta}\left(m_{1} m_{2}\right)}{\left(m_{1} m_{2}\right)^{1 / 2-i t}}\left(\frac{K}{4 \pi}\right)^{-2 \delta-2 i t} \frac{K}{4} \int_{0}^{\infty} \Phi(u) u^{-2 \delta-2 i t} d u\right\}\right] \\
& +\mathcal{O}\left(K^{1-\vartheta+\varepsilon}\right) \\
= & : \mathcal{S}_{1}+\mathcal{S}_{2}-2 \operatorname{Re} \mathcal{S}_{3}+O_{\varepsilon}\left(K^{1-\vartheta+\varepsilon}\right) .
\end{aligned}
$$

Now, following the same proof as in Hough [9, §5], for $t \ll K^{\theta}$, we obtain

$$
\frac{\mathcal{A}\left(\left\{|L M(1 / 2+\delta+i t, f)|^{2}\right\} ; K, \Phi\right)}{\mathcal{A}(K, \Phi)} \ll 1 .
$$

This completes the proof of the lemma.

6.3. Lemma. Let $\vartheta, \Upsilon, M$ be as in Theorem 6.1. If $\delta>1 / 2+\varepsilon$, then

$$
\frac{\mathcal{A}\left(\left\{|L M(1 / 2+\delta+i t, f)-1|^{2}\right\} ; K, \Phi\right)}{\mathcal{A}(K, \Phi)} \ll_{\varepsilon} M^{-2(1-\Upsilon)(\delta-(1 / 2+\varepsilon))}
$$

for any $\varepsilon>0$.

Proof. From the shape of the mollifier 5.2 , we can deduce that

$$
L M(s, f)=1+\mathcal{O}\left(M^{(1-\Upsilon)(1+\varepsilon-\operatorname{Re}(s))}\right),
$$

if $\operatorname{Re}(s)>1+\varepsilon$ for any $\varepsilon>0$. The lemma immediately follows from the above estimate.

6.4. REMARK. In fact, by 5.2 , for $\delta \geq \frac{1}{2}+\frac{9 \log \log M^{1-\Upsilon}}{\log M^{1-\Upsilon}}$ and the choice $M=K^{1-5 \vartheta}$, as in 5.41 , we have

$$
L M(1 / 2+\delta+i t, f)=1+\mathcal{O}\left(\frac{1}{\log K}\right) .
$$

Indeed, let $b_{f}(n):=\sum_{n=\ell m} \lambda_{f}(m) a_{f}(\ell) F_{\Upsilon, M}(\operatorname{rad}(\ell))$, then $L M(s, f)=\sum_{n=1}^{\infty} b_{f}(n) / n^{s}$ if $\operatorname{Re}(s)>1$. Note that we have $b_{f}(1)=1, b_{f}(n)=0$ if $2 \leq n \leq M^{1-\Upsilon}$, and $b_{f}(n) \ll$ $\sum_{n=\ell m} \tau(m) \tau(\ell) \ll \tau^{3}(n)$ for all $n \in \mathbb{N}$. So for $\delta \geq \frac{1}{2}+\frac{9 \log \log M}{(1-\Upsilon) \log M}$, we have

$$
\begin{aligned}
\operatorname{LM}(1 / 2+\delta+i t, f)-1 & \ll \sum_{n=M^{1-\Upsilon}}^{\infty} \frac{\tau^{3}(n)}{n^{1 / 2+\delta}} \ll \sum_{n=M^{1-\Upsilon}}^{\infty} \frac{\tau^{3}(n)}{n^{1+9 \log \log n / \log n}} \\
& \ll \sum_{n=M^{1-\Upsilon}}^{\infty} \frac{\tau^{3}(n)}{n(\log n)^{9}} \ll \int_{M^{1-\Upsilon}}^{\infty} \frac{d t}{t(\log t)^{9}} \sum_{n \leq t} \tau^{3}(n) \ll \frac{1}{\log K} .
\end{aligned}
$$

Consequently, we may take $W_{1}=1+\frac{10 \log \log K}{(1-\Upsilon) \log K}$ in Selberg's lemma.

We would like to thank Soundararajan for sending his unpublished paper with Conrey [3]. The following lemma is strongly based on [3]. 
6.6. LEMmA. Let $\vartheta, \Upsilon, M$ be as in Theorem 6.1. If $\frac{\log \log K}{\log K} \ll \delta \leq 1 / 2+\frac{10 \log \log K}{(1-\Upsilon) \log K}$, then for any $0<a<2(1-\Upsilon)$, we have

$$
\frac{\mathcal{A}(\{L M(1 / 2+\delta+i t, f)\} ; K, \Phi)}{\mathcal{A}(K, \Phi)}=1+\mathcal{O}_{B, a}\left((1+|t|)^{B} M^{-a \delta}\right),
$$

for some constant $B>0$ depending only on $\vartheta$.

Proof. In the region $\operatorname{Re}(s)>1$, by 5.2 we may write

$$
\operatorname{LM}(s, f)=\sum_{n=1}^{\infty} \frac{1}{n^{s}}\left(\sum_{a b c^{2}=n} \lambda_{f}(a) \lambda_{f}(b) \mu(b) \mu(b c)^{2} F_{\Upsilon, M}(b c)\right) .
$$

Using the Hecke relations we see that

$$
\sum_{a b c^{2}=n} \lambda_{f}(a) \lambda_{f}(b) \mu(b) \mu(b c)^{2} F_{\Upsilon, M}(b c)=\sum_{a b c^{2}=n} \sum_{d \mid(a, b)} \lambda_{f}\left(\frac{a b}{d^{2}}\right) \mu(b) \mu(b c)^{2} F_{\Upsilon, M}(b c),
$$

and if we set $a=\alpha d, b=\beta d$, and $g=c d$, this becomes

$$
\sum_{g^{2} \mid n} \lambda_{f}\left(\frac{n}{g^{2}}\right) \sum_{\alpha \beta=n / g^{2}} \mu(\beta g)^{2} F_{\Upsilon, M}(\beta g) \sum_{c d=g} \mu(\beta d)=\lambda_{f}(n) \sum_{\alpha \beta=n} \mu(\beta) F_{\Upsilon, M}(\beta),
$$

since the terms with $g>1$ are easily seen to disappear. Thus

$$
L M(s, f)=\sum_{n=1}^{\infty} \frac{\lambda_{f}(n)}{n^{s}} c(n), \quad \text { where } c(n)=\sum_{d \mid n} \mu(d) F_{\Upsilon, M}(d) .
$$

We have $c(1)=1 ; c(n)=\sum_{d \mid n} \mu(d)=0$ for $1<n \leq M^{1-\Upsilon}$; and $|c(n)| \leq \tau(n)$ for $n>M^{1-\Upsilon \text {. }}$

We will first handle the case $\operatorname{Re}(s)=1 / 2+\delta_{0}$ where $\delta_{0}=1 / 2+\frac{10 \log \log K}{(1-\Upsilon) \log K}$. Put $B(s, f):=\operatorname{LM}(s, f)-1$. We consider

$$
\frac{1}{2 \pi i} \int_{3-i \infty}^{3+i \infty} \Gamma(w) B(w+s, f) X^{w} d w
$$

where $X=K^{2-\vartheta}$. We shift the line of integration to $\operatorname{Re}(w)=-\delta_{0}+\delta_{1}$, where $\delta_{1}=$ $\frac{\log \log \log K}{\log K}$. The pole at $w=0$ gives $B(s, f)$, and so we conclude that

$$
\begin{aligned}
B(s, f) & =\frac{1}{2 \pi i} \int_{3-i \infty}^{3+i \infty} \Gamma(w) B(w+s, f) X^{w} d w-\frac{1}{2 \pi i} \int_{-\delta_{0}+\delta_{1}-i \infty}^{-\delta_{0}+\delta_{1}+i \infty} \Gamma(w) B(w+s, f) X^{w} d w \\
& =: T_{1}(s, f)-T_{2}(s, f) .
\end{aligned}
$$

We first estimate the contribution of the $T_{2}(s, f)$ terms. By Cauchy's inequality and Lemma 6.2 for some constant $B>0$ we have

$$
\begin{aligned}
\frac{\mathcal{A}\left(\left\{\left|T_{2}(s, f)\right|\right\} ; K, \Phi\right)}{\mathcal{A}(K, \Phi)} \ll X^{-\delta_{0}+\delta_{1}} \frac{\mathcal{A}\left(\left\{\int_{-\delta_{0}+\delta_{1}-i \infty}^{-\delta_{0}+\delta_{1}+i \infty}|\Gamma(w)||B(w+s, f)||d w|\right\} ; K, \Phi\right)}{\mathcal{A}(K, \Phi)} \\
\ll X^{-\delta_{0}+\delta_{1}} \int_{\left(-\delta_{0}+\delta_{1}\right)}|\Gamma(w)| \frac{\mathcal{A}(\{|L M(w+s, f)|\} ; K, \Phi)}{\mathcal{A}(K, \Phi)}|d w|+X^{-\delta_{0}+\delta_{1}}
\end{aligned}
$$




$$
\begin{aligned}
& \ll(1+|t|)^{B} X^{-\delta_{0}+\delta_{1}} \ll(1+|t|)^{B} K^{-2(1-2 \vartheta) \delta_{0}} \\
& \ll(1+|t|)^{B} M^{-2(1-\Upsilon) \delta_{0}} .
\end{aligned}
$$

It remains now to estimate the $T_{1}$ contribution. Since $\frac{1}{2 \pi i} \int_{(\alpha)} \Gamma(w)(X / n)^{w} d w=e^{-n / X}$, we see that

$$
T_{1}(s, f)=\sum_{n=2}^{\infty} \frac{\lambda_{f}(n) c(n)}{n^{s}} e^{-n / X}=\sum_{M^{1-\Upsilon}<n \leq X(\log K)^{2}} \frac{\lambda_{f}(n) c(n)}{n^{s}} e^{-n / X}+\mathcal{O}\left(K^{-B}\right) .
$$

In order to bound $\frac{1}{\mathcal{A}(K, \Phi)} \mathcal{A}\left(\left\{T_{1}(s, f)\right\} ; K, \Phi\right)$, for $M^{1-\Upsilon}<n \leq X(\log K)^{2}$ we consider

$$
\frac{\mathcal{A}\left(\left\{\lambda_{f}(n)\right\} ; K, \Phi\right)}{\mathcal{A}(K, \Phi)}=\frac{1}{\mathcal{A}(K, \Phi)} \sum_{k \equiv 2(4)} \Phi\left(\frac{k-1}{K}\right) \sum_{f \in H_{k}} \omega_{f} \cdot \lambda_{f}(n) .
$$

By Lemma 3.9 and Lemma 3.4, we arrive at

$$
\begin{aligned}
\frac{1}{\mathcal{A}(K, \Phi)} \sum_{k \equiv 2(4)} \Phi\left(\frac{k-1}{K}\right)\left(-2 \pi \sum_{c=1}^{\infty} \frac{S(1, n ; c)}{c} J_{k-1}\left(\frac{4 \pi \sqrt{n}}{c}\right)\right) \\
=-2 \pi \frac{1}{\mathcal{A}(K, \Phi)} \sum_{c=1}^{\infty} \frac{S(1, n ; c)}{c} \sum_{k \equiv 2(4)} \Phi\left(\frac{k-1}{K}\right) J_{k-1}\left(\frac{4 \pi \sqrt{n}}{c}\right) \\
=-2 \pi \frac{1}{\mathcal{A}(K, \Phi)} \sum_{c=1}^{\infty} \frac{S(1, n ; c)}{c}\left(\frac{1}{4} \Phi\left(\frac{4 \pi \sqrt{n}}{c K}\right)\right. \\
\left.+\frac{\sqrt{c} K}{8 \sqrt{\pi} n^{1 / 4}} \operatorname{Im}\left(e^{-2 \pi i / 8} e^{i(4 \pi \sqrt{n}) / c} \check{\Phi}\left(\frac{c K^{2}}{8 \pi \sqrt{n}}\right)\right)+\mathcal{O}\left(\frac{\sqrt{n}}{c K^{3}}\right)\right) .
\end{aligned}
$$

If $n \leq X(\log K)^{2} \ll K^{2-\vartheta / 2}$, then $\frac{4 \pi \sqrt{n}}{c K} \ll K^{-\vartheta / 4}$ and $\frac{c K^{2}}{8 \pi \sqrt{n}} \gg c K$. So $\Phi\left(\frac{4 \pi \sqrt{n}}{c K}\right)=0$; and $\check{\Phi}\left(\frac{c K^{2}}{8 \pi \sqrt{n}}\right) \ll(c K)^{-B}$ by repeated integration by parts in the definition of $\check{\Phi}$. Thus we see that if $n \leq X(\log K)^{2}$ then

$$
\frac{\mathcal{A}\left(\left\{\lambda_{f}(n)\right\} ; K, \Phi\right)}{\mathcal{A}(K, \Phi)} \ll \frac{\sqrt{n}}{K^{4}} \ll K^{-3} .
$$

Hence

$$
\frac{\mathcal{A}\left(\left\{T_{1}(s, f)\right\} ; K, \Phi\right)}{\mathcal{A}(K, \Phi)} \ll K^{-2}
$$

and

$$
\frac{\mathcal{A}\left(\left\{L M\left(1 / 2+\delta_{0}+i t, f\right)-1\right\} ; K, \Phi\right)}{\mathcal{A}(K, \Phi)} \ll_{B, a}(1+|t|)^{B} M^{-2(1-\Upsilon) \delta_{0}} .
$$

This completes the proof for $\operatorname{Re}(s)=1 / 2+\delta_{0}=1+\frac{10 \log \log K}{(1-\Upsilon) \log K}$. And the result for $\frac{\log \log K}{\log K} \ll \delta \leq 1 / 2+\frac{10 \log \log K}{(1-\Upsilon) \log K}$ now follows from Lemma 6.2 and the convexity argument.

Now we are ready to give the proof of Theorem 6.1

Proof of Theorem 6.1. Let $\delta_{2}$ be a large fixed constant. By Lemmas 6.2 and 6.3 , and a Phragmén-Lindelöf-type convexity principle for subharmonic functions which can be 
found in Kowalski [15, Lemma 25], it follows that

$$
\frac{\mathcal{A}\left(\left\{|L M(1 / 2+\delta+i t, f)-1|^{2}\right\} ; K, \Phi\right)}{\mathcal{A}(K, \Phi)} \ll_{B, \varepsilon}(1+|t|)^{B} M^{\alpha(\delta)},
$$

where $\alpha(\delta)$ is the linear function satisfying

$$
\alpha\left(\delta_{2}\right)=-2(1-\Upsilon)\left(\delta_{2}-(1 / 2+\varepsilon)\right), \quad \text { and } \quad \alpha\left(\frac{\log \log \log K}{\log K}\right)=0 .
$$

This leads to

$$
\begin{aligned}
& \frac{\mathcal{A}\left(\left\{|L M(1 / 2+\delta+i t, f)-1|^{2}\right\} ; K, \Phi\right)}{\mathcal{A}(K, \Phi)} \\
& \ll_{B, \varepsilon}(1+|t|)^{B} M^{-\frac{2(1-\Upsilon)\left(\delta_{2}-(1 / 2+\varepsilon)\right)}{\delta_{2}-(\log \log \log K) /(\log K)}}\left(\delta-\frac{\log \log \log K}{\log K}\right),
\end{aligned}
$$

for $(\log \log \log K) /(\log K) \leq \delta \leq \delta_{2}$.

Now choose $\varepsilon$ small enough and $\delta_{2}$ large enough. Combining this with Lemma 6.6. we conclude the proof of Theorem 6.1.

7. Proof of Theorem 1.9. In this section, we will prove that for at least $60 \%$ (counted with weight $\Phi$ for the sum over $k$ and with harmonic weight for the sum over forms) of the odd modular L-functions we have $L(\sigma, f)>0$ for $\sigma \in(1 / 2,1]$.

We apply Lemma 3.2 with the choices

$$
H=\frac{S}{\log K}, \quad W_{0}=\frac{1}{2}-\frac{R}{\log K}, \quad W_{1}=1+\frac{10 \log \log K}{(1-\Upsilon) \log K}, \quad \phi(s)=L M(s, f),
$$

where $R$ and $S$ are fixed positive parameters which will be chosen later. It follows that

$$
\begin{gathered}
4 S \sum_{\substack{\beta \geq 1 / 2-R / \log K \\
0 \leq \gamma \leq 2 S /(3 \log K) \\
L(\beta+i \gamma, f)=0}} \cos \left(\frac{\pi \gamma \log K}{2 S}\right) \sinh \left(\frac{\pi(R+(\beta-1 / 2) \log K)}{2 S}\right) \\
\leq I_{1}(f)+I_{2}(f)+I_{3}(f),
\end{gathered}
$$

where

$$
\begin{aligned}
& I_{1}(f):=\int_{-S}^{S} \cos \left(\frac{\pi t}{2 S}\right) \log \left|\operatorname{LM}\left(\frac{1}{2}-\frac{R}{\log K}+i \frac{t}{\log K}, f\right)\right| d t \\
& I_{2}(f):=\int_{-R}^{\left(W_{1}-1 / 2\right) \log K} \sinh \left(\frac{\pi(u+R)}{2 S}\right) \log \left|L M\left(\frac{1}{2}+\frac{u}{\log K}+i \frac{S}{\log K}, f\right)\right|^{2} d u \\
& I_{3}(f):=-\operatorname{Re} \int_{-S}^{S} \cos \left(\pi \frac{\left(W_{1}-\frac{1}{2}\right) \log K-R+i t}{2 i S}\right) \log L M\left(W_{1}+i \frac{t}{\log K}, f\right) d t
\end{aligned}
$$

Now, in the sum over zeros on the left hand side of 7.1 , the weight $\cos \left(\frac{\pi \gamma}{2 H}\right) \operatorname{can}$ be replaced by 1 . Indeed, if $\gamma=0$, we have $\cos (0)=1$; and if there is a zero $\rho=\beta+i \gamma$ 
of $L(s, f)$ with $\beta \geq \frac{1}{2}-\frac{R}{\log K}$ and $0<\gamma \leq \frac{2 S}{3 \log K}$, then we know that $\bar{\rho}$ is also a zero of $L(s, f)$, and the contribution of these two zeros is

$$
\begin{aligned}
& \geq\left[\cos \left(\frac{\pi \gamma}{2 H}\right)+\cos \left(\frac{-\pi \gamma}{2 H}\right)\right] \sinh \left(\frac{\pi(R+(\beta-1 / 2) \log K)}{2 S}\right) \\
& \geq \sinh \left(\frac{\pi(R+(\beta-1 / 2) \log K)}{2 S}\right) .
\end{aligned}
$$

It follows that

$$
4 S \sum_{\substack{\beta \geq 1 / 2-R / \log K \\ 0 \leq \gamma \leq 2 S /(3 \log K) \\ L(\beta+i \gamma, f)=0}} \sinh \left(\frac{\pi(R+(\beta-1 / 2) \log K)}{2 S}\right) \leq I_{1}(f)+I_{2}(f)+I_{3}(f),
$$

7.4. Claim. We have

$$
I_{1}(f)+I_{2}(f)+I_{3}(f) \geq \begin{cases}4 S \sinh \left(\frac{\pi R}{2 S}\right), & \text { for all } f \in H_{k} \\ 12 S \sinh \left(\frac{\pi R}{2 S}\right), & \text { if } L(s, f) \text { has a zero } \rho=\beta+i \gamma \\ & \text { with } \beta \in(1 / 2,1] \text { and }|\gamma| \leq \frac{2 S}{3 \log K} .\end{cases}
$$

Proof of Claim. Since we only consider $f \in H_{k}$ with $k \equiv 2(\bmod 4)$, it follows from Remark 1.5 that $L(1 / 2, f)=0$ for all these $f$. Hence we always know that the left hand side of 7.3 exceeds $4 S \sinh \left(\frac{\pi R}{2 S}\right)$.

Now suppose that $L(\beta+i \gamma, f)=0$ for some $\beta \in(1 / 2,1]$ and $|\gamma| \leq \frac{2 S}{3 \log K}$. If $L(s, f)$ has a zero with $\beta>\frac{1}{2}+\frac{R}{\log K}$, then the contribution from this zero to the left hand side of 7.3 would be $\geq 4 S \sinh \left(\frac{\pi R}{S}\right) \geq 8 S \sinh \left(\frac{\pi R}{2 S}\right)$, $\operatorname{since} \sinh (2 x) \geq 2 \sinh (x)$ for $x \geq 0$. Let us now assume that $L(s, f)$ has a zero with $\beta=\frac{1}{2}+\frac{\xi}{\log K}$ for some $0<\xi \leq R$. The functional equation then implies that there is also a zero with $\beta=\frac{1}{2}-\frac{\xi}{\log K}$, and together they contribute $4 S\left(\sinh \left(\frac{\pi(R+\xi)}{2 S}\right)+\sinh \left(\frac{\pi(R-\xi)}{2 S}\right)\right) \geq 8 S \sinh \left(\frac{\pi R}{2 S}\right)$ to the left hand side of 7.3. This is because the minimum value of $\sinh (x+y)+\sinh (x-y)$ for $0 \leq y \leq x$ is attained at $y=0$. Then together with the contribution from the zero at $s=1 / 2$, this proves the claim.

Let us now define

$$
\mathcal{N}_{0}(K, \Phi):=\sum_{k \equiv 2(4)} \Phi\left(\frac{k-1}{K}\right) \sum_{\substack{f \in H_{k} \\ L(\beta+i \gamma, f)=0 \\ \beta \in(1 / 2,1],|\gamma| \leq 2 S /(3 \log K)}} \omega_{f}
$$

By Claim 7.4, we have

$$
\frac{1}{2} \mathcal{A}(K, \Phi)+\mathcal{N}_{0}(K, \Phi) \leq \frac{\mathcal{A}\left(\left\{I_{1}(f)+I_{2}(f)+I_{3}(f)\right\} ; K, \Phi\right)}{8 S \sinh \left(\frac{\pi R}{2 S}\right)} .
$$

That is

$$
\frac{\mathcal{N}_{0}(K, \Phi)}{\mathcal{A}(K, \Phi)} \leq \frac{\mathcal{A}\left(\left\{I_{1}(f)+I_{2}(f)+I_{3}(f)\right\} ; K, \Phi\right)}{8 S \sinh \left(\frac{\pi R}{2 S}\right) \mathcal{A}(K, \Phi)}-\frac{1}{2}
$$


Since the weighted geometric mean is less than the weighted arithmetic mean, we have

$$
\frac{\mathcal{A}\left(\left\{\log |L M(1 / 2+\delta+i t, f)|^{2}\right\} ; K, \Phi\right)}{\mathcal{A}(K, \Phi)} \leq \log \left(\frac{\mathcal{A}\left(\left\{|L M(1 / 2+\delta+i t, f)|^{2}\right\} ; K, \Phi\right)}{\mathcal{A}(K, \Phi)}\right) .
$$

It follows that

$$
\frac{\mathcal{N}_{0}(K, \Phi)}{\mathcal{A}(K, \Phi)} \leq \frac{J_{1}(K ; \Phi)+J_{2}(K ; \Phi)}{8 S \sinh \left(\frac{\pi R}{2 S}\right)}+\frac{\mathcal{A}\left(\left\{I_{3}(f)\right\} ; \Phi, K\right)}{8 S \sinh \left(\frac{\pi R}{2 S}\right) \mathcal{A}(K, \Phi)}-\frac{1}{2},
$$

where

$$
\begin{aligned}
& J_{1}(K ; \Phi):=\int_{0}^{S} \cos \left(\frac{\pi t}{2 S}\right) \log \left(\frac{\mathcal{A}\left(\left\{\left|L M\left(\frac{1}{2}-\frac{R}{\log K}+i \frac{t}{\log K}, f\right)\right|^{2}\right\} ; K, \Phi\right)}{\mathcal{A}(K, \Phi)}\right) d t, \\
& J_{2}(K ; \Phi):=\int_{-R}^{\left(W_{1}-1 / 2\right) \log K} \psi(u) d u,
\end{aligned}
$$

$$
\text { and } \psi(u):=\sinh \left(\frac{\pi(u+R)}{2 S}\right) \log \left(\frac{\mathcal{A}\left(\left\{\left|L M\left(\frac{1}{2}+\frac{u}{\log K}+i \frac{S}{\log K}, f\right)\right|^{2}\right\} ; K, \Phi\right)}{\mathcal{A}(K, \Phi)}\right) .
$$

From now on, we shall assume

$$
S \geq \frac{\pi}{4(1-\Upsilon)(1-20 \vartheta)}
$$

We first consider $\frac{\mathcal{A}\left(\left\{I_{3}(f)\right\} ; K, \Phi\right)}{\mathcal{A}(K, \Phi)}$. By Theorem 6.1 Lemma 6.6 and 7.2, we have

$$
\frac{\mathcal{A}\left(\left\{I_{3}(f)\right\} ; K, \Phi\right)}{8 S \sinh \left(\frac{\pi R}{2 S}\right) \mathcal{A}(K, \Phi)}=\mathcal{O}_{\varepsilon}\left(K^{-\varepsilon}\right),
$$

and

$$
\frac{\mathcal{N}_{0}(K ; \Phi)}{\mathcal{A}(K, \Phi)} \leq \frac{J_{1}(K ; \Phi)+J_{2}(K ; \Phi)}{8 S \sinh \left(\frac{\pi R}{2 S}\right)}-\frac{1}{2}+\mathcal{O}_{\varepsilon}\left(K^{-\varepsilon}\right) .
$$

For $J_{2}(K ; \Phi)$, we set

$$
J_{2}(K ; \Phi)=J_{21}(K ; \Phi)+J_{22}(K ; \Phi)
$$

where

$$
\begin{aligned}
& J_{21}(K ; \Phi):=\int_{-R}^{c_{0} \log \log K} \psi(u) d u, \\
& J_{22}(K ; \Phi):=\int_{c_{0} \log \log K}^{\left(W_{1}-1 / 2\right) \log K} \psi(u) d u,
\end{aligned}
$$

and $\psi(u)$ is defined by 7.6 . By Theorem 6.1 with the choices $a=\frac{2(1-\Upsilon)(1-10 \vartheta)}{1-5 \vartheta}$ and $S=\frac{\pi}{4(1-\Upsilon)(1-20 \vartheta)}$, we have

$$
\begin{aligned}
J_{22}(K ; \Phi) & \ll \int_{c_{0} \log \log K}^{\log K} \exp \left(\frac{\pi u}{2 S}-2(1-\Upsilon)(1-10 \vartheta) u\right) d u \\
& \ll \int_{c_{0} \log \log K}^{\log K} \exp (-20 \vartheta(1-\Upsilon) u) d u \ll_{\vartheta, \Upsilon}(\log K)^{-20 \vartheta(1-\Upsilon) c_{0}} .
\end{aligned}
$$


Now by 5.41, and taking $c_{0}=\frac{S}{\pi}$, we see that

$$
\begin{aligned}
& \frac{\mathcal{N}_{0}(K, \Phi)}{\mathcal{A}(K, \Phi)} \leq \frac{1}{8 S \sinh \left(\frac{\pi R}{2 S}\right)}\left(\int_{0}^{S} \cos \left(\frac{\pi t}{2 S}\right) \log (\mathscr{V}(-R, t)) d t\right. \\
& \left.+\int_{0}^{\infty} \sinh \left(\frac{\pi u}{2 S}\right) \log (\mathscr{V}(u-R, S)) d u\right)-\frac{1}{2}+\mathcal{O}_{c}\left((\log K)^{-c}\right),
\end{aligned}
$$

for some constant $0<c \leq 20 \vartheta(1-\Upsilon) c_{0}$. Choose $c=20 \vartheta(1-\Upsilon) c_{0}$ and let $P(x), Q(x)$, $\vartheta, \Upsilon, R$, and $S$ be as in Lemma 5.43 . Then by a computer calculation of the integrals on the left hand side of 7.9 , we get

$$
\frac{\mathcal{N}_{0}(K, \Phi)}{\mathcal{A}(K, \Phi)} \leq 0.3613
$$

when $K$ is sufficiently large.

8. Proof of Theorem 1.6. In this section the variables $\vartheta, \Upsilon, R, S$, and the polynomials $P(x), Q(x)$, will be fixed as in Lemma 5.43. Let $J:=[C \log \log K]$, where $C$ is a large constant and $[x]$ means the largest integer less than $x$. Set $d:=2 S / 3$ and define the regions:

$$
\mathcal{R}_{j}:= \begin{cases}\left\{\beta+i \gamma\left|\beta \geq \frac{1}{2}+\frac{j d}{\log K},\right| \gamma \mid \leq \frac{(j+1) d}{\log K}\right\}, & \text { if } 1 \leq j \leq J-1, \\ \left\{\beta+i \gamma\left|\beta \geq \frac{1}{2}+\frac{J d}{\log K},\right| \gamma \mid \leq 1\right\}, & \text { if } j=J,\end{cases}
$$

and the zero counting sum

$$
\mathcal{N}_{j}(K, \Phi):=\sum_{k \equiv 2(4)} \Phi\left(\frac{k-1}{K}\right) \sum_{\substack{f \in H_{k} \\ L(s, f) \text { has at least } \\ \text { one zero in } \mathcal{R}_{j}}} \omega_{f}, \quad \text { if } 1 \leq j \leq J .
$$

For $1 \leq j \leq J-1$, let $\mathcal{B}_{j}$ be the rectangular box with vertices $W_{0, j} \pm H_{j}$ and $W_{1} \pm H_{j}$, where

$$
W_{0, j}:=\frac{1}{2}+\frac{j d / 2}{\log K}, \quad H_{j}:=\frac{3(j+1) d / 2}{\log K} .
$$

By Lemma 3.2 and the argument in $\$ 7$ we have

$$
\begin{aligned}
4 H_{j} \sinh ( & \left.\frac{\pi j}{6(j+1)}\right) \frac{\mathcal{N}_{j}(K, \Phi)}{\mathcal{A}(K, \Phi)} \\
\leq & \frac{\mathcal{A}\left(\left\{4 H_{j} \sum_{\begin{array}{c}
\beta+i \gamma \in \mathcal{B}_{j} \\
L(\beta+i \gamma, f)=0
\end{array}} \cos \left(\frac{\pi \gamma}{2 H_{j}}\right) \sinh \left(\frac{\pi\left(\beta-W_{0, j}\right)}{2 H_{j}}\right)\right\} ; K, \Phi\right)}{\mathcal{A}(K, \Phi)} \\
\leq & \int_{0}^{H_{j}} \cos \left(\frac{\pi t}{2 H_{j}}\right) \log \left(\frac{\mathcal{A}\left(\left\{\left|L M\left(W_{0, j}+i t, f\right)\right|^{2}\right\} ; K, \Phi\right)}{\mathcal{A}(K, \Phi)}\right) d t \\
& +\int_{W_{0, j}}^{W_{1}} \sinh \left(\frac{\pi\left(u-W_{0, j}\right)}{2 H_{j}}\right) \log \left(\frac{\mathcal{A}\left(\left\{\left|L M\left(u+i H_{j}, f\right)\right|^{2}\right\} ; K, \Phi\right)}{\mathcal{A}(K, \Phi)}\right) d u \\
& +\mathcal{O}_{\varepsilon}\left(K^{-\varepsilon}\right) .
\end{aligned}
$$


Consequently

$$
\begin{aligned}
\frac{\mathcal{N}_{j}(K, \Phi)}{\mathcal{A}(K, \Phi)} \leq & \frac{1}{6(j+1) d \sinh \left(\frac{\pi j}{6(j+1)}\right)} \\
& \times\left[\int_{0}^{(3 / 2)(j+1) d} \cos \left(\frac{\pi t}{3(j+1) d}\right) \log (\mathscr{V}(j d / 2, t)) d t\right. \\
& \left.+\int_{0}^{\infty} \sinh \left(\frac{\pi u}{3(j+1) d}\right) \log (\mathscr{V}(u+j d / 2,3(j+1) d / 2)) d u\right] \\
& +\mathcal{O}_{c}\left((\log K)^{-c}\right) .
\end{aligned}
$$

Let $c=20 \vartheta(1-\Upsilon) c_{0}$ as in $(7.9)$. By a computer calculation of the integrals on the right hand side of 8.2 , we obtained the following bounds:

$$
\begin{aligned}
\frac{\mathcal{N}_{1}(K, \Phi)}{\mathcal{A}(K, \Phi)} \leq 0.19441, \quad \frac{\mathcal{N}_{2}(K, \Phi)}{\mathcal{A}(K, \Phi)} & \leq 0.03891, \quad \frac{\mathcal{N}_{3}(K, \Phi)}{\mathcal{A}(K, \Phi)} \leq 0.00989, \\
\sum_{j=4}^{13} \frac{\mathcal{N}_{j}(K, \Phi)}{\mathcal{A}(K, \Phi)} & \leq 0.00439,
\end{aligned}
$$

provided $K$ is sufficiently large.

To obtain similar bounds for $14 \leq j \leq J-1$, we will, instead, use Lemma 5.43. Now together with the fact $x \leq \sinh (x) \leq e^{x}$ for all $x>0$, we have

$$
\begin{aligned}
\frac{\mathcal{N}_{j}(K, \Phi)}{\mathcal{A}(K, \Phi)} \leq & \frac{\frac{3}{2}(j+1) d}{6(j+1) d \sinh \left(\frac{\pi j}{6(j+1)}\right)} e^{-j d / 4} \\
& +\frac{\int_{0}^{\infty} \exp \left(\frac{\pi u}{3(j+1) d}\right) \exp (-(u+j d / 2) / 2) d u}{6(j+1) d \sinh \left(\frac{\pi j}{6(j+1)}\right)}+\mathcal{O}_{c}\left((\log K)^{-c}\right) \\
\leq & \frac{3(j+1)}{2 \pi j} e^{-j d / 4}+\frac{1}{\pi j d} \int_{0}^{\infty} e^{\pi u /(3(j+1) d)} e^{-u / 2-j d / 4} d u+\mathcal{O}_{c}\left((\log K)^{-c}\right) \\
\leq & \frac{45}{28 \pi} e^{-d j / 4}+\frac{4}{\pi d j} e^{-d j / 4}+\mathcal{O}_{c}\left((\log K)^{-c}\right) \\
\leq & \frac{3}{5} e^{-d j / 4}+\mathcal{O}_{c}\left((\log K)^{-c}\right)
\end{aligned}
$$

Hence we have

$$
\sum_{14 \leq j \leq J-1} \frac{\mathcal{N}_{j}(K, \Phi)}{\mathcal{A}(K, \Phi)} \leq \frac{3}{5} \sum_{j=14}^{\infty} e^{-d j / 4}+\mathcal{O}_{c}\left(\frac{C \log \log K}{(\log K)^{c}}\right) \leq 0.01212
$$

for suitable choice of $C$. Note that by Hough [9, Theorem 1.1], we have

$$
\frac{\mathcal{N}_{J}(K, \Phi)}{\mathcal{A}(K, \Phi)} \ll(\log K)^{-c}
$$


when $C$ is large enough. The choice of $C$ depends on the absolute constant $\theta$ in Hough [9. Theorem 1.1] but is not really important for our results. Thus by $(7.10,(8.3),(8.4)$, and 8.5 , we get

$$
\frac{\mathcal{N}(K, \Phi)}{\mathcal{A}(K, \Phi)} \leq \sum_{j=0}^{J} \frac{\mathcal{N}_{j}(K, \Phi)}{\mathcal{A}(K, \Phi)} \leq 0.63
$$

when $K, C$ are large enough.

It immediately follows from the above that

$$
\mathcal{M}(K, \Phi)=\mathcal{A}(K, \Phi)-\mathcal{N}(K, \Phi) \geq 0.27 \cdot \mathcal{A}(K, \Phi)
$$

By [8] and Goldfeld-Hoffstein-Lieman [6] one may obtain the upper bound:

$$
\Phi\left(\frac{k-1}{K}\right) \omega_{f}=\Phi\left(\frac{k-1}{K}\right) \frac{\zeta(2)}{(k-1) / 12} \cdot \frac{1}{L\left(1, \operatorname{sym}^{2} f\right)} \ll \frac{\log K}{K} .
$$

On the other hand, we have already shown the asymptotic formula (see 5.40 )

$$
\mathcal{A}(K, \Phi)=\frac{K}{4} \widetilde{\Phi}(1)+\mathcal{O}\left(K^{-B}\right) .
$$

It now easily follows from Definition 4.1 and $(8.6),(8.7), 8.8$ that the number of $f \in H_{k}$ with $k \equiv 2(\bmod 4)$ such that $L(s, f)$ has no zero $\rho=\beta+i \gamma$ with $\beta \in(1 / 2,1]$ and $|\gamma| \leq \beta-1 / 2$ will be $\gg K^{2} / \log K$.

Acknowledgments. Bingrong Huang would like to thank Jianya Liu and Wei Zhang for their valuable advice and constant encouragement and Peter Sarnak for helpful conversations. He also thanks the Department of Mathematics at Columbia University for its hospitality. The authors would also like to thank Zeev Rudnick for helpful comments.

Dorian Goldfeld is partially supported by NSA Grant H98230-16-1-0009. Bingrong Huang is supported in part by NSFC grant 11531008 and IRT_16R43 from the Ministry of Education, China. Bingrong Huang also thanks the China Scholarship Council for supporting his studies at Columbia University.

\section{References}

[1] V. Blomer, R. Khan, M. Young, Distribution of mass of holomorphic cusp forms, Duke Math. J. 162 (2013), 2609-2644.

[2] J. B. Conrey, K. Soundararajan, Real zeros of quadratic Dirichlet L-functions, Invent. Math. 150 (2002), 1-44.

[3] J. B. Conrey, K. Soundararajan, Real zeros of L-functions of modular forms, private communication.

[4] G. Csordas, T. Norfolk, R. Varga, The Riemann hypothesis and the Turán inequalities, Trans. Amer. Math. Soc. 296 (1986), 521-541.

[5] R. Godement, H. Jacquet, Zeta Functions of Simple Algebras, Lecture Notes in Math. 260, Springer, Berlin, 1972.

[6] D. Goldfeld, J. Hoffstein, D. Lieman, An effective zero free region, appendix to [8], Ann. of Math. (2) 140 (1994), 177-181. 
[7] D. Goldfeld, J. Hundley, Automorphic Representations and L-Functions for the General Linear Group, Vol. 1,2, Cambridge Stud. Adv. Math. 129, 130, Cambridge Univ. Press, Cambridge, 2011.

[8] J. Hoffstein, P. Lockhart, Coefficients of Maass forms and the Siegel zero, with an appendix by D. Goldfeld, J. Hoffstein and D. Lieman, Ann. of Math. (2) 140 (1994), 161-181.

[9] B. Hough, Zero-density estimate for modular form L-functions in weight aspect, Acta Arith. 154 (2012), 187-216.

[10] H. Iwaniec, Topics in Classical Automorphic Forms, Grad. Stud. Math. 17, Amer. Math. Soc., Providence, 1997.

[11] H. Iwaniec, E. Kowalski, Analytic Number Theory, Amer. Math. Soc. Colloq. Publ. 53, Amer. Math. Soc., Providence, 2004.

[12] H. Iwaniec, W. Luo, P. Sarnak, Low lying zeros of families of L-functions, Inst. Hautes Études Sci. Publ. Math. 91 (2001), 55-131.

[13] J. Jung, On the sparsity of positive-definite automorphic forms within a family, with an appendix by Jung and Sug Woo Shin, J. Anal. Math. 129 (2016), 105-138.

[14] R. Khan, Non-vanishing of the symmetric square L-function at the central point, Proc. Lond. Math. Soc. (3) 100 (2010), 736-762.

[15] E. Kowalski, The rank of the Jacobian of modular curves: analytic methods, Ph.D. thesis, Rutgers University, 1998.

[16] LMFDB, the database of L-functions, modular forms, and related objects, http://www.lmfdb.org/intro

[17] G. Pólya, Über die algebraisch-funktionentheoretische Untersuchungen von J.L.W.V. Jensen, 7(17), 1927.

[18] G. Ricotta, Real zeros and size of Rankin-Selberg L-functions in the level aspect, Duke Math. J. 131 (2006), 291-350.

[19] P. Sarnak, Letter to E. Bachmat on positive definite L-Functions, http://publications.ias.edu/sarnak/paper/511, 2011.

[20] A. Selberg, Contributions to the theory of Dirichlet's L-functions, Skr. Norske Vid. Akad. Oslo. I. 1946 (1946), 62 pp.

[21] H. M. Stark, D. Zagier, A property of L-functions on the real line, J. Number Theory 12 (1980), 49-52.

[22] E. C. Titchmarsh, The Theory of the Riemann Zeta-Function, 2nd ed. edited by D. R. Heath-Brown, Clarendon Press, New York, 1986.

[23] Z. Yun, W. Zhang, Shtukas and the Taylor expansion of L-functions, arXiv:1512.02683 $[$ math.NT]. 
